\title{
WestVirginiaUniversity
}

THE RESEARCH REPOSITORY @ WVU

Graduate Theses, Dissertations, and Problem Reports

1999

\section{Alterations in intrinsic heart rate in endotoxemia}

Peter Drew Wearden

West Virginia University

Follow this and additional works at: https://researchrepository.wvu.edu/etd

\section{Recommended Citation}

Wearden, Peter Drew, "Alterations in intrinsic heart rate in endotoxemia" (1999). Graduate Theses,

Dissertations, and Problem Reports. 3157.

https://researchrepository.wvu.edu/etd/3157

This Dissertation is protected by copyright and/or related rights. It has been brought to you by the The Research Repository @ WVU with permission from the rights-holder(s). You are free to use this Dissertation in any way that is permitted by the copyright and related rights legislation that applies to your use. For other uses you must obtain permission from the rights-holder(s) directly, unless additional rights are indicated by a Creative Commons license in the record and/ or on the work itself. This Dissertation has been accepted for inclusion in WVU Graduate Theses, Dissertations, and Problem Reports collection by an authorized administrator of The Research Repository @ WVU.

For more information, please contact researchrepository@mail.wvu.edu. 


\title{
Alterations in Intrinsic Heart Rate in Endotoxemia
}

\section{Peter D. Wearden}

\author{
Submitted to the School of Medicine \\ West Virginia University \\ Morgantown, WV \\ In Partial Fulfillment of the Requirements \\ for the Degree of \\ Doctor of Philosophy \\ in \\ Pharmacology and Toxicology
}

1999

\author{
William W. Fleming, Ph.D. \\ Matthew A. Boegehold, Ph.D. \\ Michael G. Mawhinney, Ph.D. \\ Ronald C. Hill, M.D. \\ Gordon F. Murray, M.D. \\ David A. Taylor, Ph.D., Chair
}

Keywords: Pharmacology, Heart Rate, Intrinsic Heart Rate, Sepsis, Endotoxin, Adenylyl

Cyclase, Protein Kinase A, Myocardial Contractility 


\section{ABSTRACT \\ Alterations in Intrinsic Heart Rate in Endotoxemia}

\section{Peter D. Wearden}

After much early debate, it is now well recognized that the origin of the heart beat is myogenic, or intrinsic, to the heart. It has been felt that the intrinsic heart rate (IHR) is constant and that the observed heart rate is controlled by tonal alterations in the activity of the autonomic nervous system in response to various stimuli. Some investigators have recognized, however, that the intrinsic heart rate (IHR) can be altered in certain conditions. Sepsis, or the host response to bacterial infection, is recognized as a stress to the host that results in decreased myocardial contractility and tachycardia. Some previous work has suggested that this tachycardia may exist outside the tonal regulation of the autonomic nervous system, raising the possibility of an elevation of the IHR. Utilizing an E. coli lipopolysaccharide (LPS) endotoxin model of sepsis in the rat, this work demonstrates an elevation of the IHR in sepsis. The experimental evidence further suggests that the $\beta$-adrenoceptor participates in the induction of the elevation in IHR early in the septic process. By sixteen hours after LPS administration the $\beta$-adrenoceptor is, however, no longer required for the ongoing elevation of IHR. It is also demonstrated that the elevation in IHR is more prolonged than the contractile dysfunction induced by LPS. 


\section{$\underline{\text { Dedication }}$}

To Andrea, Andrew, Mara and Luke

Without your support this would not have been possible Without your unconditional love this would all be meaningless 


\section{Acknowledgements}

My Committee: William W. Fleming, Ph.D.

Matthew A. Boegehold, Ph.D.

Michael G. Mawhinney, Ph.D.

Ronald C. Hill, M.D.

Gordon F. Murray, M.D.

David A. Taylor, Ph.D., Chair

My Coworkers: $\quad$ Linda Vona-Davis, Ph.D.

Kathleen Thayne

My Teachers: $\quad$ From my parents, to kindergarten teacher and on, it is through their selfless efforts that their student has advanced. Please know that your pupil recognizes that his achievements honor you.

My Mentors: $\quad$ Dr. Fleming and Dr. Taylor both of whom have my admiration. They set a standard of excellence to strive for.

Dr. Murray, my mentor as a physician and surgeon, a gentleman in the truest since of the word. To whom I will forever be indebted for his support, encouragement and advice. 


\section{Contents}

$\underline{\text { Topic }} \quad \underline{\text { Page }}$

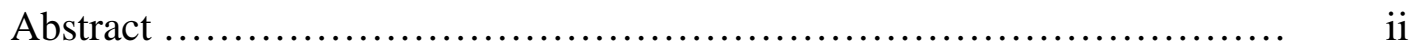

Dedication............................................................

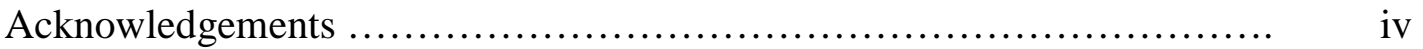

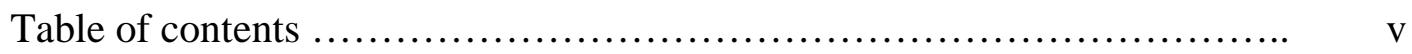

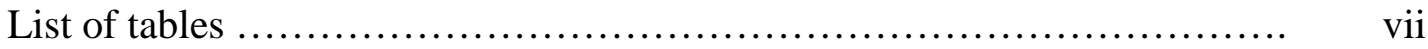

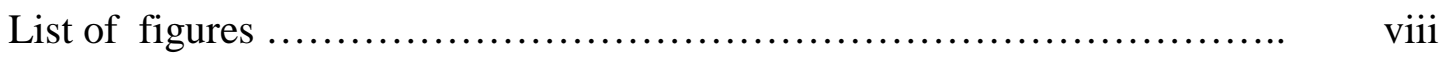

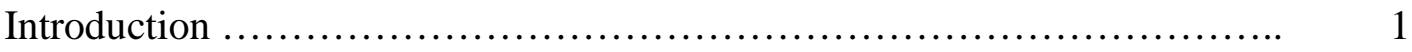

$\begin{array}{ll}\text { Historical observations on heart rate } & 6\end{array}$

$\begin{array}{ll}\text { Intrinsic cardiac rate regulation } & 14\end{array}$

Increases in right atrial pressure $\quad 18$

$\begin{array}{ll}\text { Atrial natriuretic peptide } & 19\end{array}$

Hypertension 20

Exercise 22

Alterations in body temperature $\quad 23$

$\begin{array}{ll}\text { Age } & 23\end{array}$

Miscellaneous $\quad 24$

Myocardial function in sepsis $\quad 26$

The septic process $\quad 26$

Experimental models $\quad 27$

Does the heart fail in sepsis? $\quad 28$

Failure of afterload and myocardial ischemia 30

Evidence for intrinsic failure of inotropic function and 31

its mechanism

Alterations in beta adrenoceptor signal transduction in sepsis $\quad 35$

Evidence for a difference in the chronotropic response 43

Physiologic importance 46

Experimental Design ......................................... $\quad 50$

$\begin{array}{ll}\text { Characterization of the septic model } & 50\end{array}$

Intrinsic heart rate $\quad 50$

In vitro heat rate $\quad 52$

Mechanism of induction of the increased intrinsic heart rate 53

Time course of changes in the catalytic subunit of PKA and

Time course of changes in heart rate variability $\quad 56$

Effect of frequency on myocardial contractile performance 57 


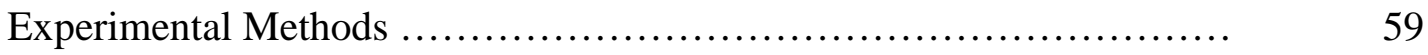

Animals $\quad 59$

$\begin{array}{ll}\text { Drugs } & 59\end{array}$

Buffer $\quad 60$

$\begin{array}{ll}\text { In vivo cardiovascular preparation } & 60\end{array}$

$\begin{array}{ll}\text { Intrinsic heart rate } & 63\end{array}$

In vitro cardiovascular preparation $\quad 64$

$\begin{array}{ll}\text { Isolated right atria preparation } & 66\end{array}$

$\begin{array}{ll}\text { Western blots } & 67\end{array}$

$\begin{array}{ll}\text { Heart rate variability } & 69\end{array}$

$\begin{array}{ll}\text { Statistical Analysis } & 71\end{array}$

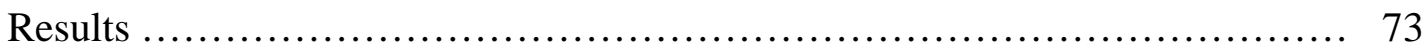

Characterization of the model

In vivo cardiovascular changes $\quad 73$

Determination of intrinsic heart rate $\quad 74$

Determination of in vitro heart rate $\quad 78$

Mechanism of induction of increased intrinsic heart rate

Reserpine inhibition $\quad 84$

Inhibition of increased intrinsic heart rate by $\beta$ adrenoceptor $\quad 88$ antagonism

Response of reserpinized animals exposed to maximal doses of $\quad 98$ BAR agonist

Time course of changes in the catalytic subunit of PKA 103

Time course of changes in heart rate variability 106

$\begin{array}{ll}\text { Relationship between inotropic and chronotropic changes } & 111\end{array}$

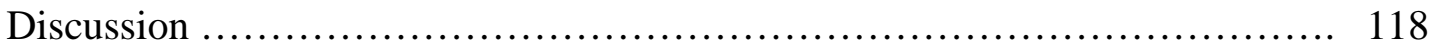

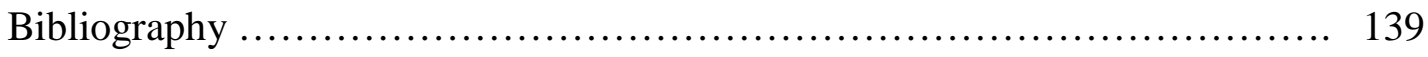

Curriculum Vitae ..................................................... 163 


\section{List of Tables}

Table 1. Animal weights 73

Table 2. Relative contributions to HR via sympathetic and parasympathetic 77 nervous systems

Table 3. In vivo HR before and after administration of hexamethonium 77

$\begin{array}{lll}\text { Table 4. In vitro HR following endotoxin administration } & 78\end{array}$

Table 5. In vitro cardiovascular parameters 16-20 hours after LPS and reserpine 86

Table 6. Mortality associated with BAAM and LPS 92

Table 7. Heart rate and mean arterial blood pressure following BAAM and LPS 93

Table 8. T-tests comparing BAAM and LPS treatment groups 94

Table 9. Coronary flow and cardiac output following BAAM and LPS 96

Table 10. Geometric mean EC50 for HR response to isoproterenol after BAAM 97

Table 11. Heart rates of isolated right atria 99

Table 12. Geometric mean EC50 for HR response of isolated right atria 101

Table 13. HR observed in vivo and in vitro at 4 and 16 hours after LPS 111

Table 14. Contractile response at 4 and 16 hours after LPS 114

Table 15. In vitro response of contractile parameters to LPS and reserpine $\quad 115$ 


\section{List of Figures}

Figure 1. Determination of intrinsic heart rate $\quad 51$

Figure 2. Effects of reserpine and propranolol 53

Figure 3. Experimental design: reserpine pretreatment 54

Figure 4. Force frequency relationship following LPS: experimental design $\quad 58$

$\begin{array}{lr}\text { Figure 5. Isolated left heart preparation } & 65\end{array}$

Figure 6. In vivo changes in heart rate and blood pressure $\quad 74$

$\begin{array}{ll}\text { Figure 7. In vivo heart rate after atropine } & 75\end{array}$

Figure 8. In vivo heart rate after propranolol 76

$\begin{array}{ll}\text { Figure 9. In vitro heart rate after LPS } & 79\end{array}$

Figure 10. Effects of anesthetic on in vitro HR 80

Figure 11. In vitro HR response to a racemic mixture of propranolol 81

Figure 12. In vitro dose response to the inactive isomer of propranolol 82

Figure 13. Response of in vitro HR to propranolol and phentolamine 83

Figure 14. In vivo HR 16-20 hours after LPS and reserpine 85

Figure 15. In vitro HR 16-20 hours after LPS and reserpine 85

Figure 16. In vitro HR following a single dose of reserpine 87

Figure 17. Isoproterenol dose response curve eight hours after BAAM 89

Figure 18. Isoproterenol dose response curve 24 hours after BAAM 90

Figure 19. Isoproterenol dose response curve after 2 doses of BAAM 91

Figure 20. Heart rate response to LPS and BAAM 92

Figure 21. Mean arterial blood pressure response to LPS and BAAM 93

Figure 22. In vitro HR following LPS and BAAM 95

Figure 23. Isoproterenol concentration response curve following BAAM 97 
Figure 24. Isolated right atria isoproterenol response

Figure 25. Isolated right atria isoproterenol response - $\%$ of maximum

Figure 26. Decline in HR of isolated right atria

Figure 27. Decline in HR of isolated right atria - regression

Figure 28. PKA catalytic subunit western blot

Figure 29. PKA catalytic subunit levels in right atria after LPS

Figure 30. Phospho-CREB western blot

Figure 32. Heart rate variability - HR

Figure 33. Heart rate variability - Total Power

Figure 34. Heart rate variability - SDNN

Figure 35. Heart rate variability - RMSDD

Figure 38. Heart rate variability - LF

Figure 39. Heart rate variability - LF nu

Figure 40. Heart rate variability - LF/HF

Figure 41. In vitro and In vivo HR response following LPS at different time periods

Figure 42. Cardiac output 4 hours after LPS

Figure 43. Ventricular dP/dt max 4 hours after LPS

Figure 44. Cardiac output 16 hours after LPS

Figure 46. In vitro HR response 4 hours after LPS with reserpine pretreatment 
Figure 48. Potential interaction between $\beta A R$ and cytokine signals 


\section{INTRODUCTION}

The aging of our population, coupled with advances in life supporting technology, surgical techniques and pharmaceuticals have markedly advanced our ability to care for the critically ill, and thereby increased the severity of illness seen in the average patient. The increased degree of illness and invasiveness of therapy leaves these patients particularly susceptible to the development of infection. As the numbers of these patients and the length of their hospitalizations have increased, sepsis, or the overwhelming response to bacterial infection has dramatically increased. Discharge surveys over the 1980s recorded a $139 \%$ increase in the discharge diagnosis of septicemia. Sepsis is now the most common cause of death in intensive care units, and is the $13^{\text {th }}$ most common cause of death in the United States. At this time there are estimated to be 400,000 cases of sepsis and 200,000 episodes of septic shock annually, resulting in 100,000 deaths. The overall cost to the American economy is estimated at ten to 15 billion dollars per year (Bone et. al., 1992; Parrillo 1990; Quartin, 1997).

With the greater recognition of sepsis as a disease entity, research into its causes and treatment has also grown. In an attempt to better organize this research, in 1992 the American College of Chest Physicians / Society of Critical Care Medicine Consensus Conference Committee proposed a standardization of terminology pertaining to sepsis and the host response to infection (Bone et. al., 1992).

Systemic inflammatory response syndrome (SIRS): SIRS is characterized by two or more of the following: (1) temperature greater than $38^{\circ} \mathrm{C}$ or less than $36^{\circ}$; (2) heart rate greater than 90 beats per minute; (3) respiratory rate greater than 20 breaths per minute, or a $\mathrm{PaCO}_{2}$ less than $32 \mathrm{mmHg}$; and (4) white blood cell count greater than 12, 000 per cubic $\mathrm{mm}$, less than 4,000 per cubic $\mathrm{mm}$, or greater than $10 \%$ band [immature] forms.

Sepsis: The presence of SIRS in association with culture proven infection. 
Septic shock: Sepsis with hypotension despite adequate fluid resuscitation, along with the presence of manifestation of hypoperfusion, including, but not limited to, lactic acidosis, oliguria, or an acute alteration in mental status.

Multiple organ dysfunction syndrome (MODS): The presence of altered organ function in an acutely ill patient such that homeostasis cannot be maintained without intervention.

This document discarded the terms septicemia, which had referred to the presence of bacteria or their toxins in the blood, and bacteremia which refers to the presence of bacteria in the blood.

As now defined, sepsis, the host response to bacteria and their toxins, includes alterations in temperature regulation, tachycardia, tachypnea and changes in the white blood cell population (Natanson et. al., 1995). All of these findings can be made with or without the presence of live bacteria in the blood and can be precipitated by bacterial endotoxin (Bone 1992).

Despite the presence of normal to often supranormal cardiac outputs, myocardial dysfunction plays a paramount role in the outcome of patients with septic shock. After some initial debate, the existence of myocardial dysfunction throughout the septic process has now been confirmed and extensively studied in both human (Bunnell and Parrillo 1996) and animal models (Abel 1993) utilizing a variety of techniques. It is now generally believed that the heart is one of the first organs to fail in MODS. Although, as delineated in the definition of sepsis, tachycardia is easily and nearly always observed, in this disease state, very little research has focused on its origin and effect. This is not surprising because it is widely recognized that tachycardia is an integral part of the autonomic response of a challenged or stressed host, and sepsis certainly represents a formidable stress on the organism. 
To properly study the dysfunction of the heart one must clearly understand the function of the heart. Perhaps William Harvey stated it best when he noted, "it has been shown by reason and experiment that blood by the beat of the ventricles flows through the lungs and heart and is pumped to the whole body ... This is the only reason for the motion of and the beat of the heart." (Harvey, 1628). Since this time, recognition of the heart as a pump is unequivocal. The mechanical nature of the heart has allowed for a wide range of scientific investigations, and mathematical calculations of its ability to do work. With advances in molecular biology, gene expression and manipulation, and cellular biochemistry, the complexity of our understanding of cardiac function increases daily. This scientific progress not withstanding, the ultimate function of the heart is no more complicated than in the times of Harvey, its purpose is simply to pump blood to the rest of the body.

The measurement of the capabilities of any pump can be recorded by its outflow. In the case of the heart the simplest measure of outflow, and therefore pump function, is the cardiac output (CO). Cardiac output is comprised of the stroke volume (SV) multiplied by the heart rate (HR). The SV (amount of blood pumped in a single stroke) is determined by the initial tension on the muscle (preload), described by the Frank-Starling law of the heart, the opposing force which the heart must pump against (afterload) and the inherent contractile properties of the heart muscle (inotropy) (Braunwald, 1971). While the interaction between preload, afterload and the inotropic state of the myocardium complicate the study of the stroke volume, determination of heart rate, an equally important determinant of $\mathrm{CO}$, is much less complicated. At the close of the $20^{\text {th }}$ century the thrust of scientific exploration is now at the level of the cell and molecule. On this level there is no argument that the rate of passage of ions across the membrane of sinoatrial node cells ultimately determines the rate at which 
the heart beats (Berne and Levy, 1997; Katz, 1992). When examining the heart rate of a patient or scientific subject, however, the cellular basis of the heartbeat is of less import, as it is generally felt that the ultimate regulation of heart rate is under the modulatory control of the autonomic nervous system via sympathetic and parasympathetic input to the heart (Levy, 1979; Randall, 1984).

Taken as outlined above, the role of the autonomic nervous system is to increase the heart rate in order to augment cardiac output on those occasions when the organism has an increased demand for oxygenated blood. As demonstrated by the "fight or flight" response to stressful situations, tachycardia is a predominant feature of this autonomic response. After the stressful situation is resolved the heart rate returns to normal. Sepsis provides another example of a stress on the organism that elevates the heart rate. Considering sepsis as an experimental model of compensatory tachycardia, this dissertation seeks to examine some fundamental, and in the author's opinion overlooked, questions with regard to the tachycardic response. Is the tachycardia of sepsis no more complicated than a simple up and down regulation via the autonomic nervous system? Can the heart alter its own internal firing rate in response to certain situations or is it merely subservient to the autonomic nervous system, and dependent upon its continual tonal regulation? Is tachycardia solely a compensatory mechanism for the organism, or as is the case in other systems (such as in the inflammatory response (Bone, 1996)), can the uncontrolled compensation itself become pathology?

Initially the historical development of our current understanding of the regulation of heart rate and its role in pump function will be examined. An examination of previous thought on the heart's ability to regulate itself follows. An overview of the extensive work regarding cardiac function in sepsis and a brief discussion of the force-frequency relationship concludes 
the background remarks. Then focusing on a model of prolonged myocardial stress, the host response to gram negative infection, the question as to whether the intrinsic rate of the heart can be altered, a mechanism by which this occurs, and the potential impact of such an adaptation will be addressed experimentally. 


\section{Historical Observations on Heart Rate}

Returning to William Harvey's observation that the pumping of blood was "the only reason for the motion of and the beat of the heart" the emphasis here has been added, but it was certainly implied by Harvey. Prior to the publication of his treatise the function of the heart carried other significant connotations including being the seat of the soul, the source of all thought and emotion, and the very vital force that was life itself.

The earliest recorded observations of what may now be the most common diagnostic maneuver, the observation of the pulse or heart rate, are contained in the Edmund Smith Surgical Papyrus. Imhotep [c. 2980 BC] the ancient Egyptian surgeon is recorded observing the pulse of a trauma victim. "Our ancient surgeon... probes with his fingers to the interior and discovers cardiac pulsation, or as he says the "fluttering and throbbing like that on the crown of an infant's head before it has grown together.'" Imhotep, according to the papyrus, uses this simple observation of the rapidity with which the heart is beating to draw clinical conclusions. "In observing the pulse our surgeon knows that he is examining the operation of the heart, and he states that the observation of the pulse is undertaken 'in order to know the action of the heart"' (Breasted, 1930).

At this time the historical record is open as to whether Imhotep's recognition of the utility of the measurement of heart rate was propagated, or much less likely, lost for the better part of the next three millennia. What is known, is that by the dawn of Western civilization, the beating of the heart had taken on a metaphysical nature that would both hinder and invigorate subsequent study of the heart and circulation. In fact, today 5000 years after Imhotep's purely physiologic observation, we are reminded every February that for centuries "matters of the heart" involved much more than the matter of the heart. 
Curiosity with the origin and nature of the beating heart is evident in the writings of

Hippocrates [460 - $375 \mathrm{BC}]$, a teacher at the medical school in Cos, and often considered the father of medicine. He noted,

"Within the heart there burns a sort of vestal fire, so subtle that it escapes immediately through any incision made to reveal it. Air coming into the heart from the trachea and lungs falls upon the flame and is set into a violent effervescence which drives it out through the opening of the great arteries and at the same time causes the swelling known as diastole" (Hoff, 1936).

These notions of fire and air conformed nicely to the contemporary concepts as to the nature of existence. Across ancient Greece at another medical school, Aristotle [384-322 BC], Plato's greatest student, subsequently undertook the study of the beating heart. (An argument may be made here to consider Aristotle "the First Investigator" of the heart beat as he provided us with the foundations of the scientific method.) Applying the scientific process to the heart beat of embryos (as would many others), and of grown animals, he made essentially three observations. First, in the chick embryo the beating heart is visible by three days of age, much earlier than the development of any of the nervous system (Adelman, 1942). Secondly, the heart is the last organ to stop working at death (ultimum moriens). The third was a simple observation, that the heart was in the center of the body, and therefore central to all other bodily functions. From these experiments Aristotle drew the conclusion that the heart is the seat of life. Coupling this with the knowledge that heartbeat changed, qualitatively and quantitatively, with emotion he extended this conclusion to include the heart being the center of all human emotion (Doby, 1963). Beyond this Aristotle largely concurred with Hippocrates' opinion that the heart contained a motive force, or fire, that propelled "life" throughout the body. Herophilus [b $330 \mathrm{BC}]$, the grandson of Aristotle, and student of Praxagoras at Cos is credited with advancing the use of the pulse to diagnose 
disease by studying its rate, strength and rhythm. He subsequently expounded on this theory to include all manner of oddities including the relationship of the heart beat to music (Seeman, 1961). Erasistratus [310 - $260 \mathrm{BC}]$, a comtemporary of Herophilus, viewed this as nonsense and the "cult of the pulse." It was Erasistratus, however, when called to see Seleucus Nicator, the son of the Macedonian General Antiochus who had taken ill, made, at the time, one of the most notable diagnoses using the pulse. Observing a noticeable quickening of the pulse rate when the boy's stepmother entered the room, Erasistratus made the diagnosis. The boy was attracted to his young step-mother and his illness, brought on by this forbidden love, was betrayed by the "lovers pulse". Both son and step-mother were exiled and married and the heart rate took on additional importance (Doby, 1963; Seeman, 1961). Galen [131-201 AD] advanced the cause of the pulse rate as a diagnostic method, checking the pulse to determine the health of patients and the veracity of their statements. By this point there was great curiosity about the heart beat, and its diagnostic utility was now recognized. The explanation of its origin, however, clearly still rested in the metaphysical.

During the early sixteenth century alchemy was the theoretical paradigm to which most physiological observations had to conform. Sylvius [1478 - 1555], an alchemist, modified the notion of the "vestal fire" as the causative agent of heart beat. He proposed that the origin of the heart beat occurred when the alkaline venous blood met up with the acidic chyle, and bile, resulting in an "effervescence" which caused diastole (Brooks, 1972). This hypothesis was obviously somewhat problematic, and later Booerhaave [1668 - 1738] is said to have noted that, "the blood is not alkaline, nor the chyle or gall acid, and even if they were mixed together no effervescence would take place, and further they never did come together in the heart" (Hoff, 1936). Some time later, Richard Lower [1631-1691], who 
would make the first observations related to gas exchange in lungs, conducted an experiment to disprove the hypothesis of Sylvius, that some chemical nature of the blood caused the heart to beat, by replacing the blood with another fluid.

"With this object in mind I drew off through the jugular vein of a Dog almost half of its total blood volume, injecting instead through the crural vein an equal amount of beer mixed with a little wine. This procedure I repeated several times in succession until, instead of blood, the fluid coming from the vein was merely a solution with less colour than the washings of meat, or than claret several times diluted. The Heartbeat, meanwhile, became only slowly more feeble, so that practically the whole of the blood was replaced by beer before life was replaced by death" (Hoff, 1936).

Although a fascinating way to introduce beer into the laboratory, this particular experiment of Lower's was less than pivotal in our current understanding of the heartbeat and circulation.

William Harvey [1578-1657], on the other hand, with the publication of his treatise, Excercitatio Anatomica de Motu Cordis et Sanquinis in Animibalis in 1628, laid the groundwork for our current understanding of the circulation. In reviewing Harvey's work in the context of the political nature of science at the time, it becomes apparent that in addition to being a talented scientist, Harvey must also have had a certain amount of political savvy. Harvey seems to have recognized that, because of the functions attributed to the heart by Aristotle and those who followed, the nature of why the heart beats had enormous religious and therefore political implications. Rather than further breach this interface between religion and science Harvey makes the simple statement "The heart is fashioned with appropriate structures for motion, as an internal organism before the body. Being finished first, Nature wished the rest of the body to be made, nourished and perfected by it, as its work and home." He continues, in what is certainly a not so subtle acknowledgement of the "funding agencies" of the time, "The heart is like the head of state holding supreme power, 
ruling everywhere" (Harvey, 1628). Harvey's tact allowed for the dissemination of his ideas about the circulation, and while it is clear that he recognized the heart to be a pump to nourish the rest of the body with blood, it is unclear how far he intended to distance himself from the physiology of Aristotle and Galen (Webster, 1965). Along these lines it should be noted that in this treatise Harvey makes no further observations on the question of precisely why and how the heart beats.

Less than 50 years later the why of the heart beat again became a contentious issue. Thomas Willis [1621-1675] rationalized that if the heart was a muscle, which it clearly appeared to be, and from his study of anatomy it was known that muscles contracted as a result of nervous stimulation, the origin of the heart beat must therefore be nervous in nature. Willis did recognize that man could not consciously control the heart, but proposed that this was because the nerves to the heart originated in the cerebellum and the voluntary nerves of the skeletal muscle had their origins in the cerebrum (Hoff, 1936). The idea that the heart beat had its origins in the brain subsequently came to be known as the "neurogenic theory" and had a number of supporters. One such scientist, John Mayow [1643 - 1679] proposed that because the dura mater was observed to be pulsatile that this was the origin of the heartbeat. He went so far as to write, of the dura mater, "I can imagine nothing else but that this membrane like all others, is intended for the production of motion" (Mayow, 1674). Fortunately for Mayow, his observations that air consisted of two gases, one of which, later to be known as oxygen, supported life and turned the blood red, assured his place in the history of science.

There was, however, an accumulation of data that clearly demonstrated that the heart could beat regardless of nervous input. One investigator (Boyle) had kept a chick alive for 
two hours after its head had been cut off, and another (Redi) removed the entire brain of a tortoise in November and it lived until the following May. Another scientist (Savolius) reported on the chick embryo, “... by the third day of incubation there is hardly a visible vestige of a brain in a chicken; nevertheless the movement of the heart is clearly noticed; it could hardly be attributed to the nerves" (Hoff, 1936). Albrecht von Haller [1708 - 1777] concurred, teaching in his seminal lectures on physiology, that while the nerves may play some role, "there is still other causes besides than that of the nerves conducing to the motion of the heart." In discussing why the "heart never ceases from its perpetual motion", he notes,

"But to me the simplicity of nature seems very great in this matter, when the auricle is relaxed, it is directly filled by the muscular force of the continuous great vein, and so the heart also contracts itself, when, in like manner, it is irritated by the blood driven into it from the auricle" (Haller, 1777).

Haller then clearly viewed "mobility is inherent to the heart" and he felt that the origin of the heartbeat was due to irritation of the blood entering the heart. Bolstered by the teachings of Haller, investigators presented a mounting body of evidence as to the myogenic origin of the heart beat. In a historical review Fye notes,

By the middle of the $19^{\text {th }}$ century, most investigators had concluded that, like skeletal muscle, heart muscle would respond to a wide variety of stimuli such as heat, cold, trauma, and electricity." (Fye, 1987).

Although these developments recognized the origin of heart rate to be within the heart there was no consideration as to whether the heart itself could change its rate. The work of the Webers (inhibitory action of the vagus), Bezold (accelerator nerves), as well as Cannon (adrenergic factors) and Loewi (cholinergic influences) in recognizing the extrinsic influences on heart rate are well chronicled (Leake, 1962). During this same time period scientists, such as Johannes Muller, Robert Remak and Carl Ludwig, had discovered ganglia within the heart. Muller, among others, put forth the notion that the origin of the heart beat 
rested within these ganglia. This view point provided for the intrinsic nature of the heart beat but allowed the ultimate causation to be neurogenic in origin (Fye, 1987).

It was the laboratories of Michael Foster and his student Walter Gaskell that ultimately determined that rythmicity was a property of cardiac muscle and not nervous in nature. Foster commented upon the thought of those who felt that cardiac ganglia initiated the heart beat,

"Now when a physiologist in his searching after the hidden cause of some secret motion finds a ganglion, he cries 'Eureka!' and generally folds his hands as if his work were done. In the case of the heart, however, we may venture to go a little further and ask the question: in what way or by what means are these ganglia the cause of the heart's spontaneous beat? " (Geison, 1978)

Foster and his pupils through a series of experiments set out to answer this question. Gaskell ultimately conducted a series of experiments with the tortoise heart demonstrating the property of automaticity in the different regions of the heart by applying heat to see if the rate increased. His experiments lead him to determine that automaticity is myogenic in origin, and that the area of the heart with the greatest automaticity is the sinus venosus. It is important to note, however, that Gaskell observed automaticity from all regions of the tortoise heart including the ventricle.

"The automatic rhythm of the ventricle is of the same kind as that of the auricle, and therefore the sinus, so that if the latter is due to the presence of motor ganglia so too is the former, and on the other hand, if the former be due to some inherent rythmical property of the ventricular muscle, then the latter is due to somewhat better development of the same kind of property in the muscular tissue of the sinus." (Gaskell, 1881)

This agreed with Stannius' [1808-1883] earlier work using ligatures to isolate various parts of the beating heart. Now an understanding of Haller's observation of the beating chick embryo at 45 hours of incubation (as did Aristotle, Harvey and Saviolus before him) and Schnek's demonstration that when he removed the heart of a three day old chick embryo and 
kept it beating outside the body, he could increase the heart rate by increases in temperature (for a review see, Patten, 1949) was no longer dependent upon the unlikely presence of ganglia for an explanation but rested upon the intrinsic rythmicity of all cardiac cells. Together, these findings firmly established that the heart beat was "myogenic" in origin and originated in the area of the great veins (primum movens) (Eyster and Meek, 1921; Brooks, 1972). The distinction that the rythmicity of the heart originated from cardiac cells rather than ganglion cells was an important advance. The precise location of these pace determining cells of the mammal, the analog of the sinus venosus, was not described until 1907 when Keith and Flack (as part of Flack's medical school summer research project (Brooks, 1972)) published their findings describing the sinoatrial node as where "the dominating rhythm of the heart normally begins" (Keith and Flack, 1907). The automaticity of the heart was now recognized as originating in the heart itself. As Patten notes, "the excision and transection experiments reviewed make the primary myogenic character of the pulsation so clear that belaboring of this old issue is uncalled for" (Patten, 1949). The heart rate, however, was recognized to be modulated, if not entirely controlled by, extrinsic factors, ultimately to be known as the autonomic nervous system (Braunwald, 1974). The question of whether the heart can alter its "myogenic" rate remains to be answered. 


\section{Intrinsic Cardiac Rate Regulation}

Any student of cardiac function quickly recognizes that while the heart beat is myogenic in origin, the regulation of its rate falls under the auspices of the autonomic nervous system. Briefly, the autonomic nervous system consists of two major divisions, the parasympathetic and the sympathetic. The parasympathetic preganglionic nerve cell bodies lie in the vagal motor nuclei of the brainstem. Passing via the vagus nerve they synapse on the postganglionic nerve cell bodies resting within the walls of the heart (generally regarded as a fat pad immediately adjacent to the heart). Neurotransmission at this point occurs via acetylcholine binding at nicotinic receptors. The postganglionic cells project to the sinoatrial (SA) and atrioventricular (AV) nodes. Again utilizing acetylcholine as a transmitter, this time at muscarinic receptors, the function of the parasympathetic nervous system is to cause an immediate slowing of heart rate. This bradycardia is due to a change in ion channel states that both hyperpolarize and decrease the upstroke of the cardiac action potential. The sympathetic preganglionic nerves originate in the spinal cord from C7 to T6. These cells have a short projection to the paravertebral ganglia where they synapse on the postganglionic cell bodies, again utilizing nicotinic receptors. The postganglionic axons travel via the great vessels of the heart to reach both the conducting system of the heart, as well as, the ventricular muscle. Their nerve terminals release norepinephrine, which acts at $\beta 1$ adrenoceptors in both the electrical and muscular tissue of the atrium and ventricle. The effect of the sympathetic nervous system is to increase the heart rate. It does this by increasing the rate of rise of the action potential and the slope of the diastolic depolarization. To accommodate for the decrease in filling time, sympathetic stimulation also increases the rate of relaxation of the myocardium. Unlike the parasympathetic nervous system, 
sympathetic stimulation also increases the inotropic state of the myocardium by increasing the amount of calcium that enters the cytoplasm of the myocardial cell. These myocardial cell effects are somewhat slower than those following vagal stimulation, occurring over several beats (for a review of nervous control of heart rate see Berne and Levy, 1997; Katz, 1992; Levick, 1995; Levy, 1979; Randall, 1984).

As already noted, the ultimate rate of the heart is determined by ion flux across cells in the sinoatrial node, presumably as influenced by the autonomic nervous system. A full elucidation of precisely which ions, and channels, is a matter of active debate in the literature and beyond the scope of this dissertation. It should be noted, however, that there are essentially three electrophysiologic changes that could alter rate. One, a reduction in the level of membrane polarization so that the length of diastolic depolarization is shortened, lessening the time required to reach depolarization. The level of membrane polarization is a function of potassium ion movement. Secondly, the slope of the diastolic depolarization could steepen irrespective of the level of maximum diastolic potential. The slope of this line is regulated by sodium movement via the $\mathrm{I}_{\mathrm{f}}$ current, a decrease in hyperpolarizing potassium movement, and to some degree the entry of calcium via its specific channels. The third, and least likely, mechanism by which heart rate could be altered is a decrease in the threshold at which the cell depolarizes completely or fires (see Berne and Levy, 1997; Levick, 1995; Katz, 1992; Campbell et. al., 1992).

Obviously, these ionic currents can function without the input of the autonomic nervous system, although they are also modulated by nervous input. Interest in investigating how the heart functions intrinsically, without input from the autonomic nervous system has led to the development of several experimental preparations. Many early investigators 
simply cut the vagus and sympathetic nerves. This does not remove the heart from the influence of epinephrine released by the adrenal gland with sympathetic stimulation. Additionally, although the vagus is easily identified, it is somewhat more difficult to be certain of complete sympathetic denervation. This problem was solved in another way by relying on the ability to perfuse the excised heart via the aortic root. From the initial observations of Oskar Langendorff in 1895 and 1897 the isolated perfused heart preparation has played a critical role in developing our current understanding of cardiac physiology (Marsh and Shattock, 1997; Doring 1990). Langendorff used these experiments to study the cardiac function of the extirpated hearts of the pig, ox, and sheep. Langendorff's advancement, which allowed for the study of the heart removed from its neural and humoral influences, first demonstrated conclusively that the heart was capable of intrinsic regulation. Subsequent alterations in Langendorff's original procedure have included the use of perfusion buffers rather than defibrinated blood. Neely modified the procedure to form an ejecting or working left heart preparation by cannulating the left atrium as the entry point of oxygenated buffer. In this preparation the left atrium functions normally, passing buffer to the ventricle via the mitral valve. This allows for the study of cardiac output and both atrial and ventricular function (Neely et. al., 1967). A variety of cannulas and balloons can be inserted into the left ventricle of the isolated heart to study contractile function. As would be expected from the myogenic origin of heart beat, these isolated heart preparations beat regularly without any input from neural or humoral substances, and the investigator is able to control two other determinants of cardiac function, preload and afterload. In general, the utilization of these preparations has focused on the study of contractility (inotropy) rather than the study of the heart rate. Influenced by the opinions of Starling, who felt that heart rate 
was not altered by hemodynamic changes, and that heart rate itself had no effect on contractility (Knowlton and Starling, 1912; Markwalder and Starling, 1912), the heart, more often than not, is paced electrically at a constant rate. Isolated right atrial preparations also beat without stimulation and have been used to study the effect of pharmacologic interventions on heart rate but, with a few exceptions, little work has focused on a change in heart rate intrinsic to the heart.

Jose is given credit for first coining the term intrinsic heart rate in 1966, although others before him had performed similar experiments. His intent was to pharmacologically duplicate the surgical isolation of the heart of animals from neural and humoral input, in man. He reasoned that by blocking the "natural sympathetic and vagal stimuli to the heart with propranolol and atropine given together, [he would be] able to produce a state closely resembling cardiac isolation in the intact animal, without surgery and without major paralysis of the systemic circulation" (Jose, 1966; Jose and Taylor, 1969). In addition to achieving his goal of a pharmacologically isolated heart, Jose also focused on heart rate, calling the heart rate following the administration of autonomic antagonists, the intrinsic heart rate (IHR). Furthermore, he noted a lower IHR in patients with "cardiac disease" and felt this to be an indicator of the severity of disease. The publication of this technique and the subsequent findings of the author as to the effects of hypoxia (Jose and Stitt, 1969) and temperature (Jose et. al., 1970) on the IHR rekindled interest in the regulation of HR outside of the effects of the autonomic nervous system.

Cavero subsequently extended this technique of blockade of the autonomic nervous system in order to mathematically examine the contributions of both the parasympathetic and sympathetic nervous system as well as the contribution of the intrinsic heart rate and any 
interaction between the two branches (Cavero et al., 1976). The use of pharmacologic denervation to study cardiac function, particularly with regard to IHR is now an accepted technique in the literature (see as examples Brusting et. al., 1983; Bolter, 1994).

The body of literature utilizing these two techniques, extirpation and pharmacologic denervation, to study intrinsic rate regulation is small but several important problems have been addressed.

\section{Increases in right atrial pressure}

Perhaps the best known example of intrinsic rate regulation is the Bainbridge or Anrep effect. Described in simplest terms this is an increase in heart rate that occurs as a result of deformation of the right atrium. This can be elicited by physically stretching the right atrium, or by increasing volume or pressure in the right atrium and thereby eliciting a deformation of the atrium. This increase in heart rate is generally transient, but can be sustained with a sufficiently large and constant stimulus. The observation was first made in mammals by Stezinsky and von Bezold in 1867 but the effect exists in both vertebrate and invertebrate hearts and has been extensively reviewed (Jensen, 1971). Bainbridge noted that by infusing fluid and increasing the venous pressure in the anesthetized dog, a tachycardia occurred. He further observed that sectioning the vagus nerve abolished the effect and so the "Bainbridge reflex" was presumed to be due to vagal withdrawal in response to increasing right atrial pressure (Bainbridge, 1915). Anrep subsequently confirmed these findings and concurred that the tachycardia was the effect of the autonomic nervous system, and the effect was greater if the starting heart rate was slower (Anrep and Segall, 1926). Similar results were also shown in response to mechanical stretch applied to the right atrium (Brooks et. al., 1966, Kappagoda et. al., 1972). The observations of several others, however, conflict with 
the notion that this is an effect of the autonomic nervous system. The effect is present despite autonomic blockade with hexamethonium (nicotinic receptor antagonist), as well as with atropine and propranolol, (Bolter, 1994; Jensen 1971; Vatner et. al., 1974). The response is also observed in vitro (Brooks et. al., 1966; Blinks, 1956). These findings have led to the conclusion that the tachycardia is not a reflex mechanism (extra- or intra-cardiac) but is an intrinsic property of the right atrium (Brooks and Lange, 1977; Pathak, 1966). For the purposes of this discussion it should be noted that the phenomenon is readily observed in rats, although to a somewhat lesser degree (12.2\% increase in heart rate) (Pathak, 1958) and that distension of the left atrium does not have an effect (Jensen, 1971).

\section{Atrial Natriuretic Peptide}

A confounding factor in attempting to understand the role of changes in right atrial

pressure and its effect on intrinsic heart rate is the role of atrial natriuretic peptide (ANP, also known as atrial natriuretic factor (ANF)). As alluded to above, one role of the atria is to act as volume receptors, and the atria contain specialized nerves with receptor endings much like baroreceptors to carry out this function (Gauer, et. al., 1961; Levick, 1995). The release of ANP, however, is endocrine in nature and not dependent on neural mechanisms. The precipitating event for ANP release is volume or pressure changes that result in distension of the atria (Lang et. al., 1985; Ruskoaho et. al., 1986; Ledsome et. al., 1985; Metzler et. al., 1986). The release of ANP is also induced by tachycardia (Bilder et. al., 1989; Riddervold et $a l ., 1991)$. It has been suggested that this endocrine response may differ from the stretch response by requiring both elevated transmural pressure, as well as an increase in rate. The combination of these effects reduces the amount of shortening of the myocyte and this is thought to be the signal for ANP release (Cho, et. al., 1991). The distinction is germane to 
this discussion because two seemingly similar physiologic events have the opposite result with regard to the intrinsic regulation of heart rate. The known effects of ANP include natriuresis, diuresis and vascular smooth muscle relaxation all appropriate compensatory responses to an acute volume overload. Another effect of ANP, unlike the effect of stretch discussed above, is the lowering of heart rate (Atchison and Ackermann, 1990; Favaretto et. al, 1997; Portaluppi et. al., 1990). The decrease in heart rate, in addition to alterations in the autonomic nervous system, has a component that is intrinsic to the heart (Ackermann et. al., 1988). This effect is not mediated directly by ANP, however, because ANP administered directly to isolated atria or hearts does not alter heart rate (Hiwatari et.al., 1986; Atchison and Ackermann, 1990). Therefore, by an unknown mechanism, ANP released in response to volume overload, decreases the observed intrinsic heart rate.

\section{Hypertension}

In the spontaneously hypertensive rat (SHR) an increased heart rate observed at two weeks of age has been demonstrated, with pharmacologic blockade, to be the result of an intrinsic elevation in heart rate (Tucker and Johnson, 1984). These animals are known to have increased sympathetic tone and the hypothesis was considered that an increase in sympathetic tone alone would increase IHR. It has subsequently been demonstrated that a long term increase in sympathetic activity or $\beta$-adrenoceptor stimulation is not sufficient to increase the IHR (Nyalander and Dahlstrom, 1984, Machado et. al., 1987). Recently, it has been shown that the increase in IHR in the SHR rat remains at four weeks of age and that there are no differences in catecholamine levels between SHR and WKY rats at this time point (Dickhout and Lee, 1998). 
Angiotensin II, a humoral factor whose circulating levels are increase in some forms of hypertension, administered over three hours will increase the rat heart rate by $27 \%$ despite antagonism of the autonomic nervous system (Machado et. al., 1987). This is in agreement with in vitro studies that have demonstrated a positive chronotropic effect of angiotensin II, which was present even in reserpinized guinea pigs (Nakashima et. al., 1982). Although Machado refers to this as an increase in "intrinsic" heart rate, there is no evidence to suggest that the heart rate would remain elevated if angiotensin II were removed from the preparation or if AII receptors were blocked. Nor has it been demonstrated that the in vitro rate of an isolated heart or right atrium is elevated following angiotensin exposure in vivo. In a onekidney-one-clip (1K1C) model of renal hypertension an interesting observation has been made. By seven days after surgical intervention $1 \mathrm{~K} 1 \mathrm{C}$ animals had developed an elevation in IHR that was not present at four days. Some of these animals were also subjected to sinoaortic deafferenation (SAD) prior to $1 \mathrm{~K} 1 \mathrm{C}$ as a means of increasing sympathetic drive. Although animals receiving both treatments (SAD and $1 \mathrm{~K} 1 \mathrm{C})$ had an elevated in vivo, or basal, heart rate, the IHR was lower than control. The authors interpreted this to mean that the increase in IHR in hypertensive rats was dependent on an intact baroreflex and that increased sympathetic drive actually lowered IHR (Machado and Brody, 1989). One possible explanation of their findings is that when increased sympathetic tone is combined with the $1 \mathrm{~K} 1 \mathrm{C}$ model of hypertension there may have been less activation of the reninangiotensin system. If angiotensin II is responsible for the increase in IHR then SAD would abolish it.

Although it appears that there is an increase in heart rate in the development of hypertension, and that this is independent of the autonomic nervous system, it remains 
unclear if the elevation in the IHR is dependent on the continual presence of elevated levels of angiotensin II.

\section{Exercise}

Resting bradycardia in a well trained individual is a widely recognized adaptation of the circulatory system. Initially this was thought to be due to changes in the input of the autonomic nervous system to the heart, a decrease in sympathetic input or an increase in vagal tone, and possibly a decrease in the number of $\beta$-adrenoceptors. Utilizing the pharmacologic determination of intrinsic heart rate, it was determined that an intrinsic bradycardia was also present (Bolter et.al., 1973; Hughson et.al., 1977; Lewis et. al., 1980; Lin and Horvath, 1972; Sutton et. al., 1967). As was observed in the example of hypertension, the intrinsic heart rate determined in this fashion alone does not exclude the possibility that a humoral substance, which acts via a mechanism other than the $\beta$ adrenoceptor or muscarinic receptors, could be influencing heart rate. In this instance, however, the lowering of the intrinsic heart rate following exercise training has also been demonstrated in isolated atria and hearts, thus ruling out the possibility of a humoral influence (Bolter and Atkinson, 1988; Nylander et. al., 1982; Schaefer et. al., 1992). Of particular interest, those rats who have undergone a chemical sympathectomy prior to training do not develop a lowering of their intrinsic heart rate, and $\beta$-adrenoceptor blockade with propranolol also prevents its development (Nylander et. al., 1982; Harri and Kuusela, 1982). The concentration response relationship of isolated atria and hearts to the $\beta$ adrenoceptor agonists, isoproterenol and norepinephrine, demonstrates no changes in maximal response or sensitivity of response to agonist (Bolter and Atkinson, 1988; Schaefer et. al., 1992). Nor is there a change in responsiveness to atropine or a combination of 
atropine and norepinephrine (Bolter and Sanders, 1988). The current data support the hypothesis that training produces a lowering of the intrinsic heart rate via mechanisms that do not depend upon the continual presence of a humoral substance, and furthermore, appear to be mediated by the sympathetic nervous system.

\section{Alterations in body temperature}

Changes in body temperature can also affect the intrinsic rate of the mammalian heart. This phenomenon was first observed by utilizing the isolated heart model (Knowlton and Starling, 1912). The findings have been confirmed by pharmacologic method of determination of intrinsic heart rate in rats (Walsh, 1969) and in both nonhuman primates (Gorman and Proppe, 1984) and humans (Jose et. al., 1970). In both primate experiments, up to $40 \%$ of the tachycardia observed was due to intrinsic changes in automaticity and there was a consistent increase in heart rate, by approximately seven beats per minute per degree centigrade temperature increase, to a plateau of 40 degrees. Lowering of the temperature of the heart below normal leads to a slowing of the heart rate, a principle frequently relied upon in cardiac surgery. Paradoxically, the long-term adaptation to heat stress includes a bradycardia that is also mediated by changes in the intrinsic heart rate (Horowitz and Meiri, 1993).

Age

One of the first findings made utilizing pharmacologic antagonism to determine the IHR was that this entity decreased with age in humans (Jose and Collison, 1970). This finding has been confirmed in man (Craft and Schwartz, 1995) and observed in both dogs and rats, including isolated rat atria and hearts (Kreider et. al., 1984; Schmidlin et. al., 1992) 


\section{Miscellaneous}

A number of other factors have been reported to have chronotropic effects that are not dependent upon the autonomic nervous system. Male rats have been shown to have a lower intrinsic heart rate than females (Chandler and DiCarlo, 1998). Among neuropeptides, neuropeptide Y (NPY) and Somatostatin, Nociceptin (Giuliani et. al., 1997), and Endothelin (Zhu et. al., 1997) have been reported to decrease heart rate, while vasoactive intestinal peptide (VIP), calcitonin gene related peptide (CGRP) (Huttemeier et.al, 1993), Neurotensin, and Histidine have been observed to exert positive chronotropic effects (for reviews see Armour et.al., 1992; Corr, 1991). Recently, adrenomedullin, released from the adrenal gland in stressful situations, has been correlated with an increased heart rate (Hirata, 1996; Nishio et. al., 1997). Obviously, changes in ion concentration and even osmolality can have a direct impact on heart rate. Hyperkalemia can arrest the heart and hyponatremia is associated with an increase in the intrinsic heart rate (Bush and Vollmer, 1983).

This list is not all inclusive, and is growing with each year. For most of the agents reported to affect heart rate the investigation of their mechanism of action is incomplete. These findings, however, do raise fundamental questions with regard to the regulation of heart rate. Is a change in heart rate simply reflective of an alteration in blood pressure or some other stimulant of the autonomic nervous system, with reflex effects on heart rate? Is heart rate adjusted outside of the autonomic nervous system? It is important to note that of all the mechanisms discussed above that modify intrinsic heart rate, only exercise-training and age have been found to exert a lasting modification. That is to say that while the others may modify heart rate outside of the autonomic nervous system, they have not been proven to modify heart rate in a fashion that outlasts their physical presence in the blood or bathing 
medium. Only exercise and age have been demonstrated, via both pharmacologic blockade and in vitro preparations, to "reset" the intrinsic heart rate. The possibility of a prolonged alteration, or resetting, of the intrinsic heart rate in sepsis will be the focus of the remainder of this discussion. 


\section{Myocardial Function in Sepsis}

The cause of death in patients with septic shock has been attributed to one, or more, of three processes. Refractory hypotension caused by peripheral vasodilatation that is unresponsive to vasopressors, myocardial depression, or multiple organ failure (Parker et. al., 1987). The second, myocardial depression or myocardial failure, is often a component of the third, and has been the subject of much experimental interest. Essentially two questions needed to be addressed to determine the nature of cardiac failure in sepsis: (1) Does the heart fail in sepsis, and if so, when? (2) What is the nature of the myocardial failure? Remembering that the heart functions as a pump, the answer to the second, the nature of the myocardial failure, could have one of several origins. These include: (a) failure of preload via a decrease in venous return; (b) a failure of afterload resulting in such profound hypotension that the coronary arteries are not perfused; (c) a failure of inotropy via one of several possible mechanisms, including depressive effects on normal cellular function, loss of ionic gradients or aberrant responses to normal regulatory signals. Furthermore, a change in any of these results in a chain of events ultimately effecting the others.

\section{The septic process}

A brief examination of the septic process and the experimental techniques used to induce sepsis in animal models illustrates some of the difficulty previous investigators have had in determining the answer to these questions. The reader will recall that sepsis is now defined as SIRS as a result of proven infection. Bacteremia is the presence of bacteria in the blood, and endotoxemia is the presence of endotoxin in the blood. It should also be recalled that gram negative bacteria contain a lipopolysaccharide (LPS) outer wall, and that endotoxin and 
LPS are synonymous terms. Composed of lipid A, repeating oligosaccharide $\mathrm{O}$ antigens and a polysaccharide core, LPS has been identified, via injecting only this component of the bacteria, as the pathogenic mechanism of gram negative infection, and that all of the systemic manifestations of sepsis are present following endotoxemia (Natanson et. al., 1986). It is the lipid A portion that conveys toxicity and the $\mathrm{O}$ antigen represents bacteria to bacteria variability. Generally, it is believed that LPS binds to LPS binding protein (LBP) produced by the liver and circulated throughout the body. This complex then binds to CD 14 receptors of immune cells, precipitating the inflammatory response including the production of cytokines particularly tumor necrosis factor $\alpha$ (TNF- $\alpha$ ), interleukin- 1 (IL-1) and interleukin6 (IL-6), although it is recognized that a host of other cytokines are also produced. (Abel, 1993; Redl et. al., 1994; Rietschel and Wagner, 1996). LPS can also stimulate endothelial and epithelial cells by a mechanism that is not fully described. There also appears to be a soluble CD14 receptor that binds LPS resulting in the stimulation of these cells without the involvement of LBP (Rietschel, et. al., 1996; Ulevitch and Tobias, 1995). Endotoxin does not seem to be directly damaging to the heart. What is more likely is that the sequelae of the inflammatory response, particularly the cytokines precipitate the observed cardiac dysfunction (Parker and Adams, 1979; Muller-Hocker and Haerty, 1993).

\section{Experimental Models}

Sepsis and septic shock can be induced experimentally in many ways and this has led to a great deal of confusion in the literature. Live or dead bacteria can be injected directly into the blood, the peritoneum or subcutaneous spaces. Endotoxin can be administered in the same fashion by bolus injection, continuous infusion or via osmotic mini pumps. In an attempt to better duplicate human sepsis some investigators have tried cecal ligation and 
puncture, the i.p. injection of fecal inoculum or the implantation of infected clots (Chaudry et. al., 1993, Spitzer, 1993). A menagerie of animals has also been used including mice, rats, guinea pigs, pigs, sheep, dogs, baboons and horses. Depending upon which method is utilized the septic response takes a varying amount of time to develop. For instance bolus injection of endotoxin results in a rapid drop in blood pressure which reaches its lowest point at eight minutes. The septic response to intraperitoneal endotoxin, or cecal ligation and puncture (CLP) takes much longer to develop. With intraperitoneal administration (LPS 5 $\mathrm{mg} / \mathrm{kg}$, i.p.) plasma endotoxin levels do not peak until two hours and remain elevated for at least 5 hours. Intravenous injection of the same dose results in an initial peak of plasma levels followed by a continuous gradual decrease (Bahrami et. al., 1993). Some animal models employ large doses of intravenous endotoxin which leads to immediate hypotension and a precipitous drop in cardiac output resulting in the death of the animal in several hours (Chaudry et. al., 1993). Other potentially compounding factors include, whether the animals are anesthetized or not, and which species was used. The experimental model used has particular relevance in understanding the literature and in terms of extrapolating the results to human disease. In most cases humans are not exposed to a single overwhelming bolus of endotoxin, but rather a more constant slow exposure over a period of time, or intermittent exposure such as with bacterial lyses with each antibiotic dose.

\section{Does the heart fail in sepsis?}

Experimentally addressing the two questions above as to when the heart fails, and what is the nature of the failure, presented unique experimental challenges exacerbated by the variety of models used. Due to the presence of normal to supranormal cardiac outputs the recognition of whether or not the heart failed in sepsis was delayed for a considerable period 
of time. A decrease in cardiac output is not generally observed until shortly before the demise of the host. Initially, it was felt that cardiac failure was a component only of terminal sepsis and was referred to as "cold-shock." At this stage the skin was cold and clammy and there was a weak pulse. The counterpart of "cold-shock" was known as "warm-shock", an earlier period in sepsis when the skin was warm and there was a bounding pulse coupled with hypotension (Waisbren, 1951; Weil and Nishijima, 1978).

As was discussed earlier, to truly examine the function of the heart as a pump the investigator must control the influencing factors, preload, afterload, inotropy and heart rate. Unfortunately, all of these parameters are significantly altered in sepsis. Circulating blood volume and preload decrease as a result of "capillary leak", afterload is profoundly altered by pronounced vasodilatation induced in part by nitric oxide (Thiemermann 1997), and heart rate is significantly increased except during the agonal period. Tachycardia and decreased afterload increase cardiac output, which can obscure decreases in contractility. Decreases in preload can make the normal heart appear dysfunctional due to a reduction in end diastolic volume. Another important consideration is what measurements of cardiac function are used, i.e. in vivo measurements compared to in vitro models. Cardiac output, left ventricular change in pressure over time, left ventricular developed pressure or a host of other derived measures have all been proposed as appropriate measures of cardiac function (Krosl and Pretorius, 1993). One of the first groups to investigate this problem demonstrated increased contractility following the induction of sepsis in the dog and related cardiac failure to circulatory deficits (for review see Raymond, 1990). Goodyear, in an early study conducted in mongrel dogs, suggested that at three hours after salmonella administration the only cardiac dysfunction "is primarily determined by peripheral mechanisms." He did consider 
the possibility that the hypoperfusion could cause "cumulative myocardial damage" (Goodyear, 1967). Gilbert, in a 1960 review, stated that there was no evidence of an alteration in the heart's ability to perform work and therefore the effects must be vascular (Gilbert, 1960). This thought was reinforced by Hinshaw and others when they demonstrated no adverse cardiac effect between one and three hours after endotoxin administration (Hinshaw et. al., 1972a).

Solis and Downing were the first to demonstrate myocardial failure before the agonal, or terminal period following endotoxin administration (Solis and Downing, 1966) and it was shortly thereafter confirmed in early human sepsis (Siegel et. al., 1967). Many of the early discrepancies had to do with the length of the experimental protocol. In the same year as his previous report, Hinshaw was able to document myocardial dysfunction three to six hours after endotoxin (Hinshaw et. al., 1972b). Krosl demonstrated in a sublethal sheep model of endotoxemia that decreases in contractility took four hours to appear (Krosl et. al., 1994). Myocardial failure at some time point was now recognized as a major component of sepsis, though the mechanism underlying this failure was unclear.

\section{Failure of afterload and myocardial ischemia}

A way in which circulatory failure could contribute to myocardial dysfunction outside of changes in preload would be myocardial ischemia. This led to one of the first hypotheses advanced that suggested that the myocardium failed in sepsis because of hypoperfusion and ischemia. The reasoning was that if the heart muscle was inadequately supplied with oxygen, due to a decrease in perfusion pressure and increased demand, the myocardium would become globally ischemic and inotropy would suffer. Animal models generated conflicting data. Some investigators found decreased coronary perfusion in dogs (Elkins et. al., 1973; 
Kleinman et. al., 1980). In contrast, experiments in pigs and cats showed a decrease in coronary vascular resistance and an augmentation of coronary blood flow (Shapiro et. al., 1985; Lefer, 1979). It has since been demonstrated in both animals and humans, that coronary perfusion is preserved in sepsis. Furthermore, there is little lactate production and the fractional extraction of arterial oxygen decreases, suggesting the oxygen supply is adequate (Adams et. al., 1990; Cunnion et. al., 1986; Dhainaut et. al., 1987).

\section{Evidence for intrinsic failure of inotropic function and its mechanism}

Eventually, evidence began to accumulate that changes in the periphery were not the proximal cause of the myocardial dysfunction. With the advent of the pulmonary artery catheter to measure cardiac preload in humans it became recognized that the "cold shock" was really a combination of hypovolemia and sepsis induced myocardial dysfunction (Cunnion and Parrillo, 1989). Even if the animal or patient was fully fluid resuscitated, however, the inotropic properties of the septic heart were not equal to control (Goldfarb, 1985, Sugi, 1991). It was now recognized that there could be some degree of inotropic failure of the heart and measurements of its ability as a pump $(\mathrm{CO})$ remain elevated, i.e. hyperdynamic, due to the markedly decreased afterload.

Some of clearest evidence of an intrinsic decrease in the inotropic properties of the heart came from in vitro experiments on hearts of animals exposed to endotoxin in vivo. These experiments allow the investigator to precisely control all determinants of pump function, preload, afterload and heart rate, leaving only inotropy as the tested variable. Parker and Adams demonstrated a decrease in the rate of tension development in isolated atrial and ventricular muscle strips of guinea pig following LPS administration (Adams, 1984; Parker and Adams, 1981). Others, using the isolated rat heart after exposure to intraperitoneal LPS, 
or endotoxin administration via osmotic minipumps, confirmed a $25-50 \%$ reduction in contractile performance early in the septic insult (Fish et. al, 1985; McDonough et. al., 1984; Romanosky et. al., 1986).

Now that it was clear there was myocardial failure in sepsis, that it occurred relatively early in the process, and that it was related to a decrease in contractility, investigation was directed toward determining the mechanism. A considerable amount of effort has been directed toward identifying a humoral myocardial depressant factor (MDF). Using cultured neonatal rat myocytes, Parillo demonstrated a decrease in the extent and velocity of myocyte shortening when the cells were exposed to the serum of septic humans with decreased ejection fractions. Serum of recovering patients did not have any depressant effect on the myocytes (Parrillo et. al., 1985). A number of investigators advanced the hypothesis that MDF had its origins in the pancreas (Lefer, 1979). Others concluded that because blood from dogs in septic shock did not depress the myocardial function of normal dogs, and that pancreatectomy did not prevent myocardial failure, that there was unlikely to be any MDF (Hinshaw, 1979).

Electrolyte and fluid disturbances have also been proposed as a mechanism of myocardial failure. Utilizing electron microscopy swelling of the myofibrillar elements and mitochondria has been shown (Coalson et. al., 1972). Others have shown an increase in the myocardial interstitial space and a decrease in the collagen content of rat hearts in sepsis (Gotloib et. al., 1992; Yu et. al., 1997). Several studies have shown that there is an increase in cellular sodium and a decrease in cellular potassium concentrations in skeletal muscle during sepsis or endotoxemia (Chiao et. al., 1992; Karlstad and Sayeed, 1987). In agreement with these findings, Liu's group has correlated decreased sodium-potassium pump function 
and internalization of the sodium-potassium pump with a decrease in cardiac performance during sepsis (Liu, 1990; Tang et. al., 1993) and Schornack showed a 25\% decrease in pump translocase activity (Schornack et. al., 1997). Archer has suggested that insulin improves cardiac function in sepsis by increasing intracellular potassium (Archer et. al., 1978). Conversely, increased phosphatase activity of the $\mathrm{Na}+-\mathrm{K}+\mathrm{ATPase}$ pump activity has been demonstrated in skeletal muscle (Jacobs et. al., 1991) and digoxin, which inhibits the pump, has been reported to partially reverse myocardial dysfunction in sepsis (Coalson et. al., 1972; Narasaway et. al., 1989). No attempt has been made to reconcile these contradictory findings. Compounding the issue, an increase in free cystolic calcium has been implicated as the cause of cardiac failure in sepsis (Hotchkiss and Karl, 1996; Deaciuc and Spitzer, 1987) and impaired function of the cardiac sarcoplasmic reticulum has been demonstrated four hours after endotoxin administration to dogs (Wu and Liu, 1991). Others, however, have shown normal function of the sarcoplasmic reticulum (Kutsky and Parker, 1990;

McDonough, 1988). A normal amount of intracellular calcium coupled to a decreased ability of the myofilaments to respond to calcium, was shown in cardiac myocytes exposed directly to LPS (Yasuda and Lew, 1997). Again pointing to the discrepancies regarding the model used, guinea pigs exposed in vivo to endotoxin demonstrated decreased calcium availability and normal myofilament response (Rigby et. al., 1998).

Over the past decade a great deal of the work examining sepsis in general, and its attendant myocardial dysfunction in particular, has focused upon the role of nitric oxide (NO). It is clearly recognized that there is an upregulation of inducible nitric oxide synthase in a number of cells, including myocytes following endotoxin exposure (Johnson and Billiar, 1998; Payen et. al., 1996). Furthermore, the increased production of nitric oxide via this 
pathway is contributory, if not solely responsible for, the hypotension seen in sepsis (Robertson et. al., 1994; Thiemermann, 1997). As discussed this hypotension could jeopardize cardiac function if preload or afterload were severely altered. What is less clear, however, is whether NO has any direct or indirect effects on myocardial inotropy. While several authors have suggested that NO produces an inhibition of myocardial contraction (Brady et. al., 1993; Finkel et. al., 1992; Hebertson et. al., 1996; Weiss et. al., 1997; Kumar et. al., 1999) others have demonstrated either no effect of NO (Hock et.al., 1997; Meng et. al., 1997; Keller et. al., 1995; Klabunde and Cotson, 1995; Toth and Heard, 1997; Weyrich et. al., 1994), that TNF- $\alpha$ causes myocardial depression in the absence of NO (MullerWerdan, et. al., 1997) or that the inhibition of NO synthase has a detrimental effect on contractility (Robertson et.al., 1994). It is possible that by-products of NO formation, such as peroxynitrite could also be directly toxic to the heart. Further work is necessary to elucidate the precise role NO plays in the myocardial contractile dysfunction of sepsis. Summarizing, it is likely that there are myocardial depressive substances produced by endotoxin, such as cytokines, NO, free radicals and others, which act in concert to cause myocardial depression. Tumor necrosis factor and several interleukins are particularly likely suspects (Kumar and Parrillo, 1993). Equally as likely, is that one of the effects of these agents is an alteration in electrolyte homeostasis. At this time, however, it is unclear which agents and which ions may be responsible for these effects and there is a significant amount of contradictory information in the literature. One area of investigation into the intrinsic failure of the contractile mechanisms that is better developed, and there is slightly more consensus, is the cardiac response to catecholamines. 


\section{Alterations in beta adrenoceptor signal transduction in sepsis}

As discussed earlier norepinephrine released from sympathetic nerve terminals and epinephrine released from the adrenal medulla increase the rate and force of the heart beat acting primarily through $\beta 1$ adrenoceptors ( $\beta \mathrm{AR})$. The signal transduction mechanism of the $\beta A R$ in the heart is propagated through the $\mathrm{G}_{\text {stimulatory }}$ coupling protein which, upon the binding of ligand, becomes activated and binds and exchanges GDP for GTP. At this point the $\mathrm{G}_{\beta \gamma}$ subunit of the $\mathrm{G}$ protein is cleaved. The $\mathrm{G}_{\mathrm{s}^{-}}$GTP complex dissociates from the receptor and activates adenylyl cyclase to increase cAMP production from ATP. The activated $\mathrm{G}_{\mathrm{S}}$-GTP is short lived due to the dephosphorylation of the GTP to GDP, which allows $G_{S}$ to rebind the $G_{\beta \gamma}$ subunit. Production of cAMP is regulated via adenylyl cyclase activity, as well as, by its breakdown by phosphodiesterase. Ultimately cAMP alters the function of the cAMP dependent protein kinases resulting in changes in ion channel function and thereby cardiac function (Katz, 1992; Bohm, 1995).

With regard to sepsis, several early investigators have administered propranolol shortly after i.v. endotoxin in dogs which, in most instances, quickly precipitated the animals demise. This led to speculation that cardiac function was under, in fact dependent upon, sympathetic control (Cann, 1972; Goodyear, 1967; Levy and Blattberg, 1967). Hinshaw witnessed no survivors in adrenalectomized dogs administered endotoxin (Hinshaw et.al., 1964). During the same time period, Bhagat demonstrated that there was a decreased sensitivity, as measured by maximal tension, in guinea pig atrial muscles treated with norepinephrine after endotoxin exposure (Bhagat et. al., 1970) and Parrat demonstrated a decrease in the left ventricular $\mathrm{dP} / \mathrm{dt}$ in response to catecholamines three hours after endotoxin in the cat (Parrat, 1973). These findings, a dependence on sympathetic stimulation and a lack of 
responsiveness, to catecholamines, would at first seem contradictory but again may reflect differences in the time course and experimental model used. If early in the septic insult the host responded by increasing sympathetic drive, as would be predicted, and the cardiac response was dependent upon this to compensate, later failure of this mechanism would appear as myocardial dysfunction.

A brief review of the terminology used to describe decreases in responsiveness to ligands and the mechanisms of development would be useful at this point. Diminished responses to the same concentration of a receptor to agonist over a short period of time (minutes to hours) is referred to as desensitization or tachyphylaxis. This change in responsiveness is a common phenomenon, particularly of the $\beta \mathrm{AR}$, following exposure of cells to a high concentration of agonist. Desensitization can occur via either a homologous or heterologous mechanism. The former is hormone specific, or in other words, involves changes in particular receptors, and consists of four possible processes. (1) The receptor may be internalized (and therefore cannot bind drug). (2) The total receptor number may decrease due to enzymatic degradation also referred to as down regulation. (3) The receptor may become uncoupled from its signal transduction mechanism (in this instance the $\mathrm{G}$ protein). (4) There may be a decrease in the affinity of the receptor for agonist. Heterologous desensitization involves changes in the signal transduction pathway rather than the receptor. Examples would be G-protein changes or alterations in adenylyl cyclase activity (Harden, 1983; Fleming, 1997; Katz, 1992; Ross, 1997). These mechanisms are heterologous in that more than one receptor may utilize the same, or portions of the same, signal transduction pathways and a change here would diminish responses to all agonists that use that transduction process. In both homologous and heterologous desensitization, administration 
of an equal amount of drug after an initial exposure results in a decreased response. It would be insufficient if an attempt were not made to delineate between desensitization and subsensitivity (also called tolerance). This is a distinction often lost in the literature. As described, desensitization develops quickly and has been reported to involve changes only in the receptor or its signal transduction cascade. The most notable distinguishing characteristic between desensitization and subsensitivity is the time frame in which it develops.

Subsensitivity requires hours to days for development. Subsensitivity can also be specific, i.e. to a single hormone and receptor, but is frequently nonspecific, in that the dose response curve for any agent that elicits the experimental response is shifted to the right. (Fleming, 1997; Fleming and Westfall, 1988; Johnson and Fleming, 1989). The mechanisms producing both desensitization and subsensitivity may be similar with two notable exceptions. Changes in sensitivity often require a modification in the cellular abundance of specific proteins, hence the longer time course. The other distinction is that mechanisms have been proposed by which sensitivity is altered that do not directly relate to the receptor or its signal transduction mechanism, e.g. changes in membrane potential (for a review of these terms see Fleming and Taylor, 1995; Johnson and Fleming, 1989). The converse of subsensitivity is denoted supersensitivity (dose response curve shifts to the left), there is no widely recognized increase in responsiveness occurring over a short period of time.

Based on these descriptions and the suggestion of decreased BAR responsiveness, it is important to answer several questions with regard to sepsis. Is there an increase in sympathetic activity that could precipitate either desensitization or subsensitivity of the myocardium? What is the time course of changes in responsiveness? Where in the receptor signal transduction cascade have alterations in responsiveness occurred? 
Following the administration of endotoxin there is a pronounced response of the sympathoadrenal system. Plasma epinephrine (EPI) levels increase to over 45 times the normal level but quickly begin to return toward control values by three hours after the septic insult in the rat. Norepinephrine (NE) levels do not demonstrate as rapid , or as pronounced of an increase (approximately six times normal), but remain elevated, with a gradual diminution of the level throughout the septic course (Jones and Romano 1989, McKechnie et al 1985). Endotoxin administered to rabbits causes an immediate increase in EPI that peaks at $500 \%$ of normal at one hour, NE levels rise more slowly and are $300 \%$ of normal at 6 hours (Heiffer et. al., 1960). A similar relatively rapid rise in EPI and a slower smaller increase in NE has also been demonstrated in dogs (Nykiel and Glaviano, 1961; Spink et. al., 1966; Rosenberg et. al., 1961). Increases in plasma catecholamines are also clearly seen in human patients. It is interesting to note that, in humans, higher catecholamine levels are correlated with a worse prognosis (Benedict and Rose, 1992).

Coincidental to the increase in plasma catecholamines, is an increase in the postganglionic sympathetic nerve firing rates to the heart and spleen in cats (Halinen et. al., 1977) and to the kidney of rats (Palsson et. al., 1988). A $700 \%$ increase in the rate of NE turnover was observed in a rat model of endotoxemia (Pardini et. al., 1983). Over time, there is a depletion of NE from end organs in endotoxemia (Heiffer et. al., 1960;Pardini et al 1982). Depletion of norepinephrine from tissues can occur in one of several ways; decreased synthesis, decreased reuptake into the nerve terminal, decreased vesicularization after reuptake or increased metabolism. Decreased reuptake has been demonstrated in the spleen but not the heart of endotoxemic rats (Pardini et al, 1982). Increased neuronal metabolism of NE has been suggested in the rat and guinea pig after endotoxin exposure (Bhagat et. al., 
1979; Pardini et. al., 1982). There is also evidence from the crayfish to suggest that the number of quanta of NE released per stimulus is increased by endotoxin (Parnas et. al., 1971).

In addition to these alterations in NE release and reuptake, the initiating mechanism for the sympathetic response that leads to NE release may be altered. Hypotension or other homeostatic reflex mechanisms appear to play less of an important role and endotoxin itself, or the inflammatory response to it, may be modulating the increase in catecholamine levels. This statement is based on several experimental findings. Changes in autonomic activity in endotoxemia do not necessarily correlate with alterations in blood pressure or heart rate. Most in vivo experimental models demonstrate only a transient drop in blood pressure or no hypotension and a slower and prolonged elevation of HR which does not correlate with BP or catecholamine changes (Lang et al, 1985; Palsson, 1988; Jones and Romano, 1984, 1989). Plasma catecholamine levels become elevated to many times normal even in the sinoaortic denervated rat (Zhou et al 1991). Additionally, endotoxin has been demonstrated to cause enhanced release of catecholamines at peripheral nerve terminals in response to constant rate stimulation of the sympathetic nervous system in the pithed rat. The data from this study suggested that the rise in plasma catecholamines after endotoxin administration was partially due to a direct peripheral action of LPS on sympathetic nerve terminals, independent of increases in firing rate (Jones et al 1994). Taken together these findings point to a significant increase in plasma catecholamine levels in sepsis. This increase is of sufficient magnitude to cause $\beta$ AR desensitization and decreased responsiveness.

As already discussed alterations in agonist concentration can lead to changes in receptor populations. Several investigators have reported changes in the $\beta$ AR population in 
endotoxemia and sepsis. Endotoxin induced a decrease in $\beta$ AR number in canine lymphocytes by $41 \%$ when observed five hours after LPS injection (Silverman et. al., 1990). Guinea pig lymphocyte $\beta$ AR sites were reduced $38 \%$ four days after endotoxin exposure (Van Oosterhout et. al., 1989). In a rat model, utilizing a high dose of endotoxin (30 mg/kg), Shepherd, found there to be an approximately $12 \%$ decrease in $\beta$ AR density in the heart at three hours and observed no differences in binding affinity or between high and low affinity sites (Shepherd et. al., 1986). In a follow up experiment the same group demonstrated a $25 \%$ reduction in $\beta$ AR density of isolated myocytes four hours after a $1 \mathrm{mg} / \mathrm{kg}$ dose of endotoxin (Shepherd et. al., 1987). A 50-mg/kg endotoxin challenge in rats by another group resulted in a $41 \%$ decrease in cardiac $\beta$ AR density (Boillot et. al., 1997). Following cecal ligation and puncture, rather than i.v. endotoxin, there was a $35 \%$ increase in sarcolemmal $\left[{ }^{3} \mathrm{H}\right]$ dihydroalprenolol (DHA) binding capacity at 9 hours and a 39\% decrease at 18 hours. At this later time frame there was an increase in the light vesicle DHA binding in septic animals, suggesting receptor internalization (Tang and Liu, 1996).

Other investigators, using lower doses of endotoxin $(2 \mathrm{mg} / \mathrm{kg})$, have not observed any change in $\beta$ AR concentration or affinity of the rat heart four hours after i.v. administration (Gardey, et. al., 1994). In another study also utilizing rats, following a dose of $5 \mathrm{mg} / \mathrm{kg}$ there was no change in the maximum binding or the dissociation constant of $\left[{ }^{3} \mathrm{H}\right] \mathrm{DHA}$ at 0.5 or 3 hours after i.v. endotoxin administration. In the agonal stages of sepsis there was, however, a decrease in the affinity of isoproterenol binding. The control binding modeled to a two-state fit, whereas the endotoxin exposed animals modeled to one state of lower affinity (Romano and Jones, 1986). Isolated cardiac myocytes exposed to activated splenocyte conditioned media, which contains many of the cytokines seen in sepsis, for 72 hours showed no changes 
in $\beta$ AR population. Exposure of the cardiac myocytes to $3 \times 10^{-7} \mathrm{M}$ isoproterenol for 30 minutes resulted in a 45\% reduction in cell surface $\beta$ AR (Chung et. al., 1990).

These data further reflect and reinforce the confusion in the sepsis literature that results from the use of different species and models. There is, however, reasonable consensus that higher doses of endotoxin do result in changes in the $\beta$ AR population, likely via internalization. There is also an indication of a likely change in isoproterenol binding affinity at various time points. This is particularly important since it has been shown that changes in the coupling status between the $\beta \mathrm{AR}$ and the G-protein will alter the binding affinity of isoproterenol to the $\beta A R$ (for review see Levitski, 1988). The presence of two states of $\beta \mathrm{AR}$, binding at high and low affinity, is due to the higher affinity of the receptor for agonist when it is complexed to the $\mathrm{G}_{\mathrm{s}}$ protein. When the $\mathrm{G}_{\mathrm{s}}$ protein is uncoupled, or unable to complex to the $\beta$ AR, the receptor shows low affinity binding. Therefore, those investigators who saw an increase in the low affinity binding state proposed that there was an uncoupling of the $\beta A R$ signal transduction mechanism at the level of the $\mathrm{G}_{\mathrm{s}}$ protein. This could then account for the decrease in responsiveness to catecholamines.

Measuring the signal transduction mechanisms downstream from the G-protein would help determine if this uncoupling is responsible for the decreased responsiveness. One of the earliest experiments in this area showed a decrease in cAMP production to isoproterenol stimulation in rat ventricular slices five hours after in vivo endotoxin administration (Romano and Jones, 1985). Obviously this observation could be secondary to decreased cAMP formation via either alterations in the signal transduction or decreased activity of adenylyl cyclase itself or increased degradation of cAMP. Romanosky demonstrated a 70\% increase in basal cAMP levels of the whole rat heart three hours after a $30 \mathrm{mg} / \mathrm{kg}$ i.v. dose of 
endotoxin. Exposure of these hearts to a concentration of isoproterenol that produced a $120 \%$ increase in cAMP levels in control animals produced only a $27 \%$ increase in those treated with LPS (Romanosky et. al., 1986). In a follow-up to their earlier study, Jones and Romano, examined cAMP formation in response to isoproterenol stimulation and found that it was normal 0.5 and 3 hours after in vivo endotoxin administration. Cyclic AMP production was, however, decreased at 6 hours in response to $10^{-7} \mathrm{M}$ isoproterenol stimulation. At none of the time points were basal levels of cAMP different between exposed and control animals. The same authors also stimulated adenylyl cyclase with $\mathrm{NaF}$ and 5' guanylylimidodiphosphate [Gpp(NH)p]. Both of these agents stimulate cAMP production distal to the $\beta \mathrm{AR}$, most likely at the G-protein. When these agents were used there was no difference in adenylyl cyclase activity at any time point. This finding bolstered the idea that the G-protein could no longer couple to the receptor in the terminal stage of sepsis and this idea was corroborated by others (Campbell and Forse, 1993; Jones and Romano, 1986b). Cardiac myocytes isolated from endotoxin treated $(30 \mathrm{mg} / \mathrm{kg})$ rats three hours after administration, also showed a $75 \%$ reduction in isoproterenol stimulated cAMP production. Cyclic AMP production in response to forskolin, a direct stimulator of adenylyl cyclase was also markedly reduced. The cardiac membranes from these rats, in addition showed decreased adenylyl cyclase activity in the presence of $\mathrm{NaF}$ and $\mathrm{Gpp}(\mathrm{NH}) \mathrm{p}$ (Shepherd et. al., 1986). These effects appeared to be endotoxin dose dependent (Shepherd et. al., 1987). Isolated rat hearts perfused directly with $25 \mu \mathrm{g} / \mathrm{ml}$ of endotoxin in vitro were also reported to have a decreased response to isoproterenol but not to forskolin (Bensard et. al., 1994). When cardiac myocytes are exposed to the cytokine rich splenocyte-conditioned media, isoproterenol stimulated cAMP production begins to decrease at 48 hours when 
compared to normal media. In this experiment the defect again appeared to be at the level of G-protein as forskolin yielded equal results in both treatment groups (Chung et. al., 1990). This decreased responsiveness does not appear to be $\beta$ AR subtype specific as it has also been demonstrated for the $\beta 2$ adrenoceptor as measured by the relaxation of pig coronary arterioles (Wang et. al., 1994). Furthermore, these experimental observations extend to humans. Lymphocytes, which also have $\beta$ ARs, isolated from septic patients produce a diminished amount of cAMP in response to isoproterenol and $\mathrm{NaF}$ exposure (Silverman et. al., 1990). Although there are some differences with regard to basal cAMP levels and response to forskolin stimulation all of these experiments have identified some degree of hyporesponsiveness to $\beta$-adrenoceptor stimulation. The hyporesponsiveness appears to be related to the dose of endotoxin administered and points to dysfunction at least at the level of the G-protein if not also at the level of adenylyl cyclase. The time course of these changes, minutes to hours, conforms to the definition of desensitization.

\section{Evidence for a difference in the chronotropic response}

Curiously, two groups of researchers have reported supersensitivity of adenylyl cyclase activity in sepsis. Carli's group showed an increase in cAMP production with exposure to isoproterenol, $\mathrm{NaF}$ and $\mathrm{Gpp}(\mathrm{NH}) \mathrm{p}$ four hours after administering $2 \mathrm{mg} / \mathrm{kg}$ to the rat. It is perplexing that they were unable to demonstrate this response, and in fact saw the reverse with 1 and $3 \mathrm{mg} / \mathrm{kg}$ (Gardey et al, 1990). The authors of this report do not state which portion of the rat heart was used for their preparation. McDonough's group, however, clearly demonstrates supersensitivity only in the right atrium. Their model was to examine the heart rate response of the isolated rat heart to isoproterenol 24 hours after cecal ligation and puncture. The authors first demonstrated intrinsic myocardial contractile dysfunction as 
would be expected. Intriguingly, they also found a 58 fold increase in the sensitivity to isoproterenol exposure as measured by the agonist induced change in the beating rate of isolated right atria (Smith et. al., 1986). This finding was confirmed in a repeat experiment again using cecal ligation and puncture (Barker et. al., 1990). In this latter study, sensitivity to forskolin was also examined and found to not be increased. It should be noted that the same group, using the same model, also examined the sensitivity of the inotropic response in a Langendorff perfused left ventricle model and witnessed no changes in isoproterenol sensitivity (Smith and McDonough, 1988).

Although these findings could be dismissed as spurious, there are several distinctions which make them stand out. The McDonough group was the only group to examine the chronotropic response specifically. Nor have any other investigators examined the response of the right atria in particular. These experiments were also conducted using cecal ligation and puncture with tissue isolation at 24 hours. This model generally leads to the development of sepsis over several hours rather than several seconds as with the i.v. injection of high doses of endotoxin. The literature bears out why these may be important distinctions. First, regardless of the animal model or method of inducing sepsis tachycardia is a ubiquitous finding. Even in those studies cited above which demonstrated $\beta$-adrenergic dysfunction tachycardia was observed. This leads to an interesting paradox because the same $\beta$ adrenoceptors which mediate inotropy also mediate chronotropy. If the decreased responsiveness with regard to inotropy is related to the receptor why is there an augmentation of chronotropy? There is also evidence in the literature to suggest that the right atrium may be qualitatively different with regard to $\beta$-adrenoceptor signal transduction when compared to the ventricle and even the left atrium. The laboratory of Fleming and Taylor has shown 
differences in the development of supersensitivity between the right and left atria as well as the ventricle of the guinea pig in response to chronic reserpine treatment (Fleming, 1984; Fleming and Taylor, 1991; Roberts et. al., 1992). Recently, in a study also using rat cecal ligation and puncture, a significant increase in type II protein kinase A (PKA, also called cAMP dependent protein kinase) was observed in the right atrium that was not present in either the left atrium or ventricle (Hsu et. al., 1997). These findings raise the possibility that the $\beta$-adrenergic receptor signal transduction responsible for the chronotropic response to sepsis could be modulated differently than the inotropic response at the cellular level.

A close review of this literature reveals another interesting possibility, particularly in light of the previous discussion of the regulation of heart rate. The data of several authors provide evidence that the tachycardia of sepsis may, in part, be due to an increase in the intrinsic heart rate. In addition to observing supersensitivity of the chronotropic response to isoproterenol McDonough's group also observed an approximately $16 \%$ increase in the heart rate of isolated hearts and isolated right atrium (Smith et. al., 1986; Barker et. al., 1990; Muniz and McDonough 1991). Using osmotic minipumps, Fish observed a 29\% increase in the in vitro heart rate of LPS treated rats at six hours (Fish, 1985). Romanosky also reported a $15 \%$ increase in the in vitro heart rate but in his model this was not statistically significant. Circumstantially related to a possible increase in the intrinsic heart rate, is the observation that heart rate remains elevated after the normalization of blood pressure, and continues to increase even after catecholamine levels taper off (Lang et. al., 1985; Qi et. al., 1991; Sharma et. al., 1996). In humans the heart rate has been shown to be elevated 10 days into the septic process despite the normalization of blood pressure (Shoemaker et. al., 1993). All 
of this is suggestive that the elevation of heart rate may be intrinsic to the heart, rather than part of ongoing autonomic regulatory mechanism.

Additional evidence for alterations in the control of heart rate in sepsis have come from a relatively new area in the study of autonomic function. The interval between beats of the heart (the R-R interval on an electrocardiogram) is not constant but is subject to small fluctuations. These fluctuations are felt to be due to changes in the autonomic input to the sinoatrial node. Heart rate variability $(\mathrm{HRV})$ is then defined as the cyclic fluctuations in the heart period over time. The measurement of heart rate variability has been proposed, and extensively studied, as a quantitative measurement of autonomic nervous system activity (for review of this technique see Akselrod et. al., 1981; Kuwahara et. al., 1994; Malliani et. al., 1991; Ori et. al., 1992). Applying this technique to septic and endotoxemic humans and rabbits three separate groups of investigators have demonstrated a decrease in heart rate variability in sepsis. This is particularly interesting in that all three groups identify a decrease in the sympathetic modulation of heart rate despite the presence of a significant tachycardia (Garrard et. al., 1993; Godin et. al., 1996; Goldstein 1995). Taken together these data suggest the following hypothesis; that the tachycardia of endotoxemia in sepsis may, at least in part, be due to an elevation of the intrinsic heart rate. Before exploring this hypothesis experimentally it would be appropriate to address the potential physiologic or pathologic effects on an intrinsic elevation of heart rate in the septic myocardium.

\section{Physiological importance}

Where as Frank and Starling's observation of the relation between length and tension is widely recognized, an earlier description of the relationship between force and frequency is

less well known. At the end of the $19^{\text {th }}$ century, Bowditch first described (Bowditch, 1871) 
what perhaps most appropriately should be called the force -interval relationship. The Bowditch effect, also known as the treppe effect or positive staircase, states that any particular frequency of stimulation the heart develops a certain amount of tension. With an increase in the frequency of stimulation the tension which the heart generates rises with each successive beat until a new higher steady state is reached. This small stepwise increase in tension displayed graphically appears like a staircase hence its name. It is now known that this phenomenon is related to an increase in calcium entry into the cell. Decreasing the interval between stimulation results in increases in intracellular calcium concentration via both sarcolemmal calcium channels and release from the sarcoplamsic reticulum (for a comprehensive review of the Bowditch effect see Koch-Weser and Blinks, 1963; Noble and Seed, 1992). It is important to note that Bowditch recognized an optimal rate of stimulation above which contraction declined. Woodworth confirmed Bowditch's frog heart observations in the dog heart (Woodworth, 1902). These findings and the many that followed relied heavily on isolated tissue allowing for a focus upon inotropy. Unlike isolated muscle, the effects of increasing rate in vivo are modest at best (Seed, 1992). This is because, in general, the cardiac output of the heart remains constant. So as heart rate increases there is also a decrease in diastolic filling or preload which negates any frequency induced increase in inotropy (Levick, 1995; Ross et. al., 1965). This should not be confused with an increase in heart rate produced by sympathetic stimulation, such as with exercise. With catecholamine induced increases in chronotropy, stimulation of the $\beta A R$ in the ventricle results in an increase in the rate of relaxation that preserves diastolic filling and therefore preload. There is also augmentation of the contractile process so that with sympathetic stimulation both heart rate and stroke volume increase. The heart as a pump is 
therefore dependent upon the coordinated adjustment of several aspects of its function in order to increase cardiac output.

Even in isolated tissues a reversal of the Bowditch effect is seen in pathological conditions. In human heart failure an alteration of the interval-force relationship has been shown to occur. Isolated ventricular strips from failing myocardium at the time of transplant show a peak function at 81 beats per minute compared to 174 beats per minute in control hearts (Feldman et. al., 1988; Mulieri et. al., 1992). The negative impact of heart rate on contractility is particularly acute in dilated cardiomyopathy (Schmidt et. al., 1995; Just, 1996) which is precisely the state of the failing human heart in sepsis (Parillo, 1990). From these data the conclusion can be drawn that increases in heart rate could certainly jeopardize the cardiac pump in sepsis. This statement would be particularly true if the $\beta$ AR signal transduction in the ventricle is dysfunctional preventing a coordinated autonomic response, or if the mechanisms of handling cellular calcium turnover are deranged resulting in the over accumulation of intracellular calcium which is documented to jeopardize cell function .

Increasing the heart rate also increases myocardial oxygen demands. The heart, which in the normal state extracts $72 \%$ of the arterial oxygen delivered, has little reserve in terms of oxygen delivery (Schlant and Sonnenblick, 1994; Vatner and Braunwald, 1972). At least $15 \%$ of critically ill patients have been shown to develop myocardial ischemia which is correlated with a significantly worse prognosis (Guest et. al., 1995).

As one can now see, the origin and regulation of the heart beat has been the object of long-standing curiosity. While the heart rate is myogenic in origin it is clearly regulated by autonomic influences. There is ample evidence, however, to suggest the possibility of alterations in the intrinsic heart rate. Sepsis has been shown to negatively effect myocardial 
contractility while simultaneously increasing heart rate. There are also proven abnormalities of the autonomic nervous system and its signal transduction mechanisms in sepsis. These two processes combined result in a divergence of alterations in inotropy and chronotropy that could exacerbate myocardial dysfunction.

These points raise several questions which the following research seeks to explore: (1) Is there an elevation of the intrinsic heart rate in sepsis? (2) How is the tachycardia of sepsis initiated and maintained? (3) Is there a distinction between the regulation of inotropy and chronotropy in sepsis? 


\section{EXPERIMENTAL DESIGN}

\section{Characterization of the septic model}

As addressed in the introduction, there is little standardization of the experimental models described in the literature to examine the effects of sepsis and endotoxin. The model used in these experiments, a single dose $(5 \mathrm{mg} / \mathrm{kg})$ of Escherichia Coli (E. Coli) endotoxin 16-20 hours prior to the examination of cardiac function is relatively unique. The experimental model used for these experiments was a rat model of endotoxemia. Male Sprague-Dawley rats were used exclusively. E. Coli endotoxin $(5 \mathrm{mg} / \mathrm{kg})$ in a $2.5-\mathrm{mg} / \mathrm{ml}$ solution of $0.9 \%$ normal saline was injected intraperitoneally at four p.m. the evening before experimental observations. Data were collected between eight a.m. and 12 noon the following day hence the 16-20 hour model of endotoxemia. Control animals received an equal volume of $0.9 \%$ normal saline. Due to the unique nature of the model and the relatively distant time point, after a single endotoxin exposure, at which cardiac function was examined it was necessary to chronicle the vascular response in this model. Animals were implanted with cannulae in the femoral artery. Following an overnight recovery period, these animals were administered intraperitoneal endotoxin $(5 \mathrm{mg} / \mathrm{kg})$ and their cardiovascular response (heart rate and blood pressure) was followed for 24 hours.

\section{Intrinsic Heart Rate}

The first hypothesis tested experimentally was that there is an elevation of the intrinsic heart rate in sepsis, or more appropriately, endotoxemia. As discussed in the introduction, intrinsic heart rate can be determined in one of two fashions. The first is according to the method of Jose and extended by Cavero (Jose, 1966; Cavero et. al., 1976). This entails injecting the animal, or patient, with a sufficient dose of atropine to antagonize the muscarinic receptors of the heart. This is followed by the injection of sufficient dose of 
propranolol, or another beta blocker, to antagonize the $\beta A R$. This sequence is reversed in half of the group of animals to avoid an effect of order of administration. After the animals have received both drugs the recorded heart rate is devoid of parasympathetic or sympathetic influences and is recognized as the intrinsic heart rate (see Experimental Methods for a complete description of this procedure). As confirmation of the accuracy of this procedure hexamethonium, which antagonizes the nicotinic receptors of both the parasympathetic and sympathetic ganglia, should also pharmacologically denervate the heart. Animals treated with endotoxin or saline vehicle in the same fashion, were then exposed to hexamethonium and the heart rate was determined. The sequence of the intrinsic heart rate experiments is outlined below (figure 1)

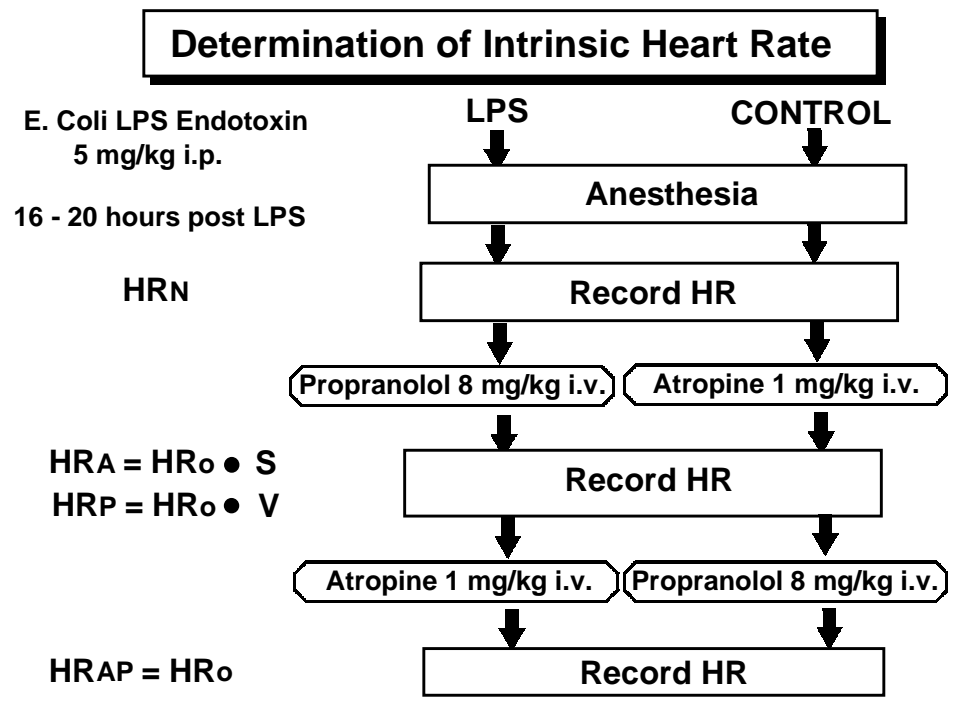

Figure 1. Experimental design to determine intrinsic heart rate. Animals had been treated 16 to 20 hours earlier with LPS $(5 \mathrm{mg} / \mathrm{kg})$. Sodium pentobarbital anesthetic $(50 \mathrm{mg} / \mathrm{kg})$ was used. Equal numbers of LPS and Control animals received propranolol or atropine first followed by the second drug. $H R N=$ initial $H R, H R A=H R$ after atropine, $H R P=H R$ after propranolol, $S=$ sympathetic influence, $V=$ parasympathetic influence, $H R o=$ intrinsic heart rate. 


\section{In vitro heart rate}

Neither of these experiments, however, remove the heart from influences not related to the muscarinic or $\beta \mathrm{AR}$ that could potentially effect heart rate. The excised or extirpated heart perfused with oxygenated buffer is removed from both neural and humoral influences. The beating rate of this preparation is also indicative of the intrinsic heart rate. Experiments were conducted carrying out the same endotoxin injection procedure as outlined above. At 16-20 hours the hearts were removed from the animal and perfused with oxygenated buffer as a working left heart preparation. After a 20 minute stabilization procedure the in vitro heart rate was recorded and followed over the next 40 minutes.

In order to exclude the possibility of spontaneous, or unstimulated, release of catecholamines from the isolated heart, some of the hearts were exposed to propranolol after the completion of the stabilization period. A range of concentrations was used. Since propranolol is recognized to have membrane stabilizing properties at relatively low concentrations (Doggrell, 1990) an experiment was therefore conducted to determine the degree to which this property was effecting the observed results. The active, in terms of $\beta$ adrenoceptor antagonism, isomer of propranolol is the levorotatory, or S (-) form. The dextrorotatory or $\mathrm{R}(+)$ form is unable to antagonize the receptor but has the same membrane stabilizing properties at similar concentrations. The isolated hearts from separate groups of endotoxin treated and untreated animals were exposed to either the inactive $R(+)$ isomer or the racemic RS mixture. This allowed for the determination of the concentration at which nonspecific, or membrane stabilizing, effects of propranolol were being observed. To rule out the possibility of an $\alpha$-adrenoceptor mediated effect some of the isolated hearts were also exposed to the non specific $\alpha$-adrenoceptor antagonist phentolamine. 


\section{Mechanism of induction of the increased intrinsic heart rate}

Catecholamine stimulation of the $\beta \mathrm{AR}$, and concomitant parasympathetic withdrawal, is the only widely recognized mechanism by which an organism increases its heart rate. As outlined in the introduction there is a significant increase in catecholamine release in sepsis. In order to determine if this catecholamine release participated in the increased intrinsic heart rate, or if it occurred by a wholly other process, the following experiments were conducted.

Following the release of norepinephrine from a nerve terminal and its stimulation of adrenoceptors any catecholamine that remains in the synapse is quickly taken up by a reuptake transporter. Following reuptake, the catecholamine is then repackaged into vesicles by a second mechanism. When this vesicular reuptake process is inhibited by reserpine, the norepinephrine is acted upon by monoamine oxidase and catecholamines are depleted by virtue of metabolism to inactive compounds (figure 2).

Figure 2. Effects of reserpine and propranolol at the sympathetic nerve terminal are depicted. With stimulation of the nerve norepinephrine (NE) is released and crosses the synapse to stimulate the beta-adrenoceptor $(\beta R)$. This is antagonized by propranolol. NE is actively taken up into the nerve and is repackaged into vesicles. Reserpine blocks the vesicular packaging. NE not in vesicles is broken down by monoamine oxidase (MAO).

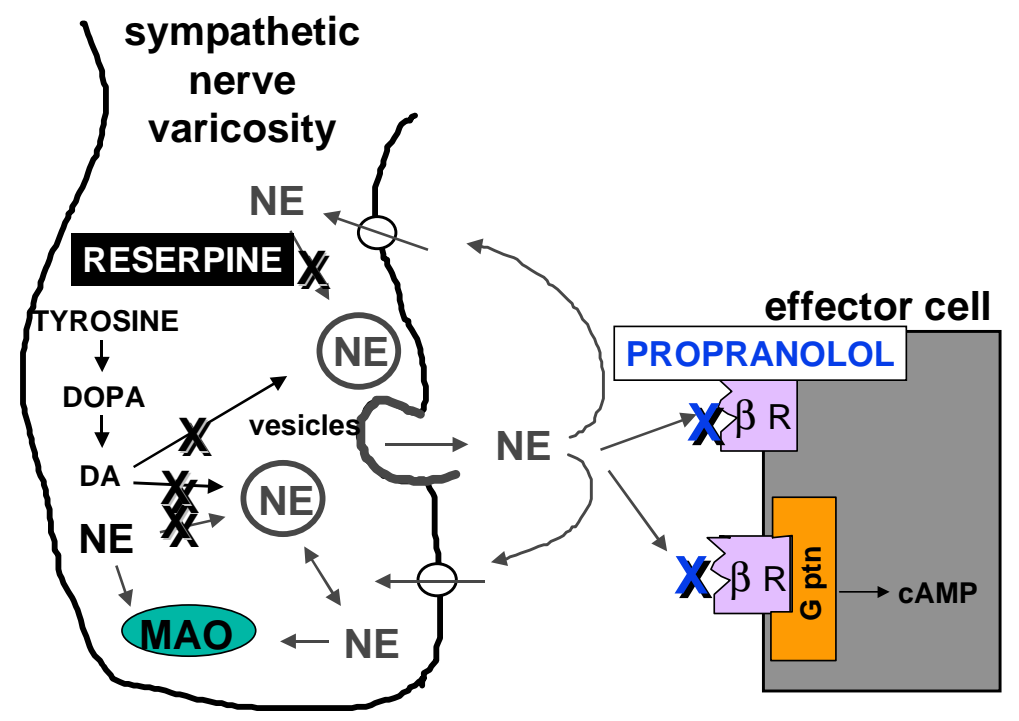


Exploiting this drug action to deplete synaptic catecholamines, animals were pretreated with reserpine and then exposed to endotoxin. The experimental design is demonstrated below (figure 3).

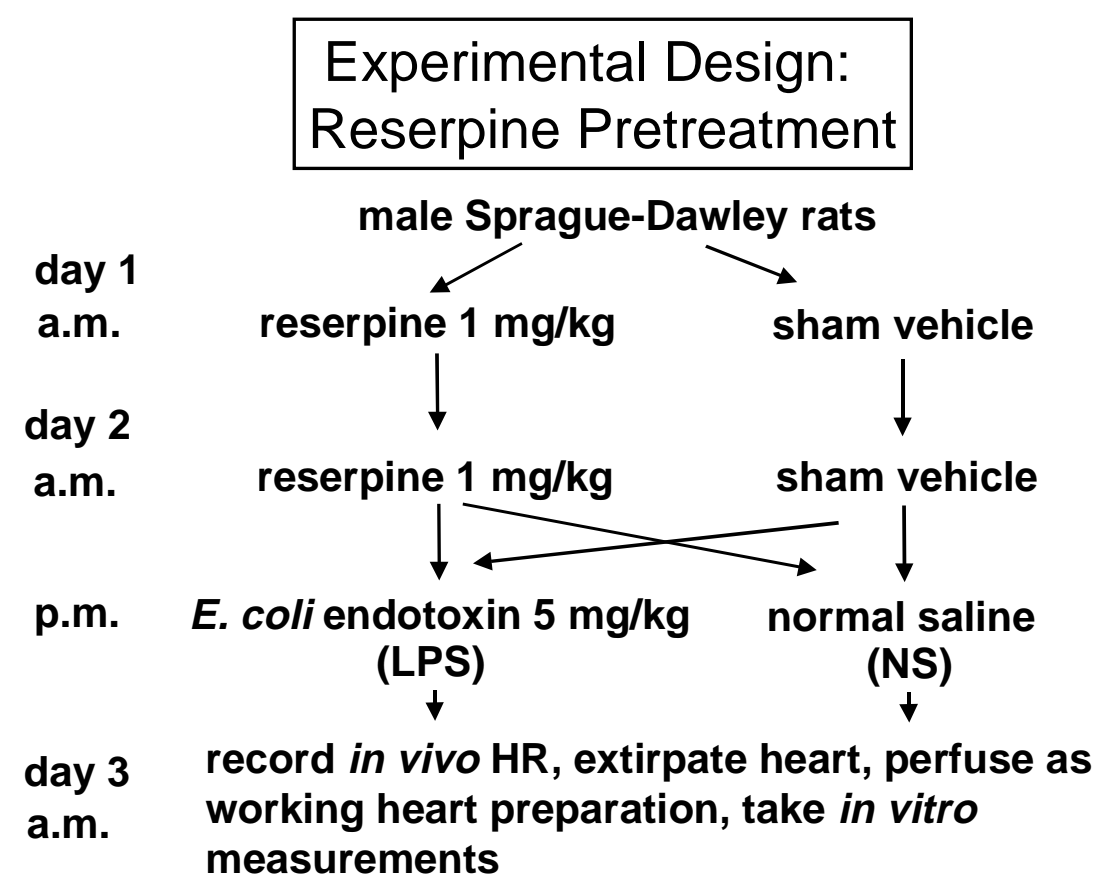

Figure 3. Design of reserpine experiments. Equal numbers of animals were pretreated with reserpine $(1 \mathrm{mg} / \mathrm{kg}$, i.p.) or sham vehicle at 9 a.m. on day one and day two. At four p.m. on day two LPS (5 mg/kg, i.p.) or normal saline (NS) was given. On day three data were collected in the a.m., 16-20 hours after LPS.

The reserpine experiment indicated that catecholamines did play a role in the intrinsic elevation of heart rate. This experiment did not rule out the possibility that reserpine affected another neurotransmitter besides the catecholamines or that reserpine had a toxic effect on the heart. It is also possible, although unlikely, that the catecholamines, which were depleted by reserpine, were increasing the heart rate via a mechanism other than activation of the $\beta$ adrenoceptor. Therefore, experiments were designed to evaluate the role of the $\beta$ adrenoceptor in the elevation of intrinsic heart rate. Bromoacetyl alprenolol menthane (BAAM) is an alkalinizing irreversible $\beta A R$ antagonist. The appropriate dose and duration 
of action of BAAM were first characterized by construction of dose response curves to isoproterenol in vivo. Once the appropriate dose antagonist had been determined the animals were pretreated with BAAM or vehicle. Thirty minutes later equal numbers of animals receiving BAAM or vehicle were exposed to endotoxin or saline. Sixteen to 20 hours later the animals were sacrificed, hearts extirpated and in vitro HR recorded. Dose response curves to isoproterenol were then performed in vitro.

An additional experiment was conducted in an attempt to answer the following question. Was the presence of LPS (or the cytokines it stimulates) required simultaneously with catecholamines to induce a change in IHR? The alternative hypothesis was that LPS induced the change in IHR but the change became manifest only after exposure to catecholamines. Presumably if the latter hypothesis were true, that catecholamines are required only for the change in IHR to become apparent, but not to induce the change, the HR of an isolated heart of an LPS treated animal exposed in vitro to a $\beta$ AR agonist would remain elevated even if the animal had been pretreated with reserpine. In order to examine the chronotropic response of atrial tissue to the $\beta 1$-adrenoceptor agonist isoproterenol and the decay of this response from its maximum it was most efficient to use a preparation that required smaller volumes of buffer. For this reason an isolated right atrial preparation was utilized. Animals were pretreated with reserpine or sham vehicle as described above. Equal numbers of each group were then exposed to endotoxin and sacrificed 16-20 hours later for a total of four treatment groups. The right atria was excised and suspended via a strain gauge recorder in a $10 \mathrm{ml}$ water bath. A 40 -minute stabilization period was observed then a cumulative concentration response curve to isoproterenol was constructed. Following the final dose of isoproterenol the bath was thoroughly rinsed with fresh buffer and was rerinsed 
every 15 minutes. The HR was recorded every 5 minutes for one hour to observe the decay in maximal isoproterenol response.

\section{Time Course of Changes in the Catalytic Subunit of Protein Kinase A and Phosphorylated CREB}

Prolonged activation of cAMP has been demonstrated to decrease the levels of the catalytic subunit of PKA is some cell types (Garrel et. al., 1995; Boundy et. al., 1998). Additionally, levels of PKA activity have been demonstrated to be variable in a rat model of sepsis depending upon the location in the heart and the duration of the septic process (Hsu et. al., 1997). CREB is a target of PKA-C phosphorylation in the nucleus (Cooper, 1997). In order to better characterize the time course of possible changes in PKA catalytic subunit and phosphorylated CREB levels in our septic model, the right atria were removed from LPS treated rats or control animals at two, four, eight and 16 hours after the onset of sepsis. The atria were then processed for western blotting. Western blots were performed and analyzed via densitometry using an antibody specific for the catalytic subunit of PKA and phosphorlyated CREB.

\section{Time Course of Changes in Heart Rate Variability}

The beat to beat interval, or instantaneous heart rate, is not absolutely constant but varies as a result of many factors including respiration, blood pressure regulation, actions of the renin-angiotensin and other known and unknown factors. The study of this variability can be approached in two manners: analysis in the time domain or frequency domain (Malik, 1996; Stein et. al., 1993). Analysis of the time domain examines simple statistical variability in the heart rate and provides information as to the total variability present over a certain period of time. Frequency domain analysis is also known as power spectral analysis. This 
method uses Fourier analysis to separate the total heart rate variability into the variability resulting from periodic oscillations at various frequencies. The resulting spectra have been demonstrated to provide information as to the amount of sympathetic and parasympathetic input to total heart rate variability (Ori et. al., 1992). These findings have been confirmed in the human and a number of experimental animals including the rat (Kuwahara et. al., 1994; Japundzic et. al., 1990). Alterations in heart rate variability have been observed in septic experimental animals as well as humans. Furthermore, these changes in heart rate variability have suggested both a worse prognosis and tachycardia in the presence of decreased sympathetic activity (Garrard et. al., 1993; Godin et. al., 1996; Goldstein 1995). In attempt to correlate our observations of an elevated intrinsic heart rate with changes in heart rate variability we utilized this technique in our experimental model. A total of ten rats were anesthetized and subcutaneous electrodes were attached to either side of the anterior chest wall. Following a 24 hour recovery period the lead I ECG tracing was monitored for 24 hours. After this control period each of the animals received $5 \mathrm{mg} / \mathrm{kg}$ of LPS i.p. and the ECG tracing was monitored for a further 24 hours. Heart rate variability in both the time and frequency domain was examined off line for a 5 minute time period of each hour.

\section{Effect of Frequency on Myocardial Contractile Performance}

Several additional questions arose about the experimental model. The first was whether, in this model, there were alterations in myocardial inotropy at 4 hours after endotoxin exposure, as many others had reported. It was also of interest to determine whether there was a decrease in inotropy 16 hours following LPS administration, the time at which the chronotropic changes were observed study. Additionally, it was desirable to examine the degree to which an increase in heart rate may impact contractile indices. The 
following experiment was designed to answer all of these questions. Animals were treated with endotoxin or saline as above. Equal numbers of animals in each group were then sacrificed at 4 or 16-20 hours after endotoxin or saline treatment. The hearts were extirpated and perfused as working left heart preparations. After a 20 minute stabilization period the in vitro heart rate was recorded as before. The hearts were then electrically paced at 300, 350, 400 and 450 beats per minute. These rates were chosen as they cover the range of physiologic heart rates observed in the rat. At each HR, cardiac output and stroke volume and change in aortic pressure over change in time $(\mathrm{A} \mathrm{dP} / \mathrm{dt})$ were recorded. The preparation was then converted to a Langendorff perfusion method in order study isovolemic contraction (see methods). At the same heart rates, the maximum change in left ventricular pressure over the change in time (LV dP/dt max) and the left ventricular developed pressure (LVDP) for a given preload were examined. This experiment is depicted graphically below (figure 4).

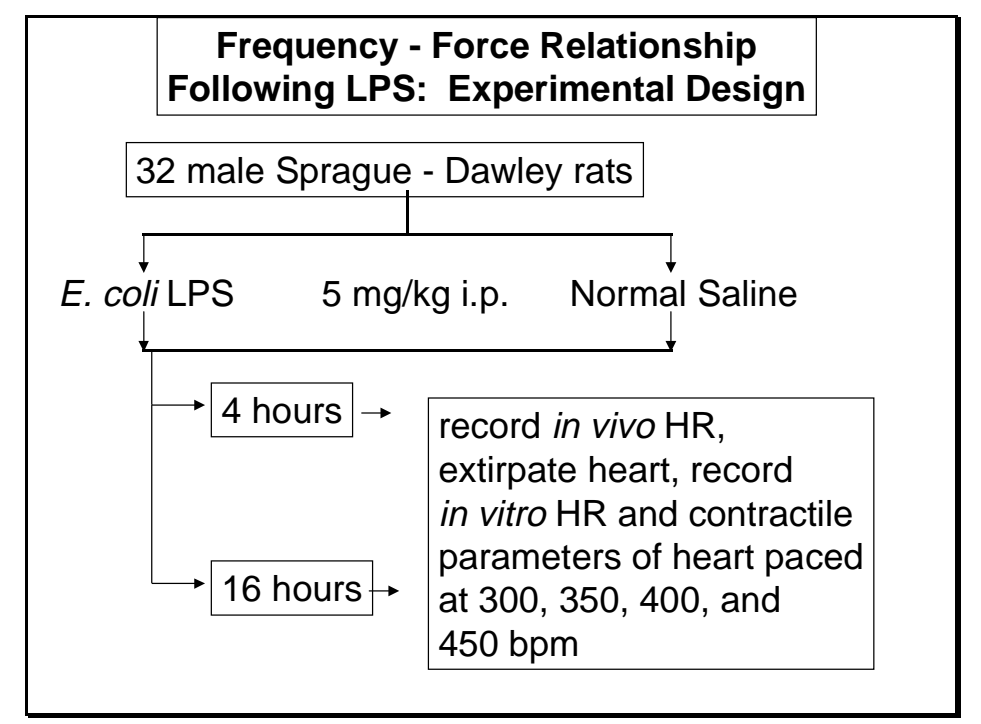

Figure 4. Diagram depicting the experimental model used to determine the effect of frequency of myocardial contractile performance after endotoxin administration at both 4 and 16 hours. Equal numbers of rats received either endotoxin (LPS) or saline in vivo. Equal numbers of rats from both groups were sacrificed at 4 or 16 hours to examine heart rate $(H R)$ and contractile indices. 


\section{EXPERIMENTAL METHODS}

Animals

Male Sprague-Dawley rats weighing between 325 and 475 grams were used in the following experiments. All animals were obtained from Hilltop Lab Animals (Scottsdale, PA). Rats were housed and cared for by the West Virginia University Office of Laboratory Animal Resources in accordance with the West Virginia University Animal Care Guidelines and Protocol Statement in compliance with the Animal Welfare Act. Animals were quarantined for 5 days after receipt and the Institutional Animal Care and Use Committee approved all procedures.

\section{Drugs}

Initially sodium pentobarbital (Nembutal Sodium Solution, $50 \mathrm{mg} / \mathrm{ml}$, Abbott Laboratories; North Chicago, IL) was used for anesthesia at a dose of $50 \mathrm{mg} / \mathrm{kg}$ intraperitoneally. At the request of the West Virginia University Animal Care and Use Committee, Telazol (Fort Dodge Animal Health; Fort Dodge, IA) was subsequently used for anesthesia. This drug consists of equal parts of tiletamine hydrochloride a dissociative anesthetic, and zolezepam hydrochloride a nonphenothiazine diazepinone, and was used at a dose of $30 \mathrm{mg} / \mathrm{kg}$. Escherichia Coli (serotype O55:B5; Sigma; St. Louis, MO) endotoxin (LPS) was prepared in normal saline at a concentration of $2.5 \mathrm{mg} / \mathrm{ml}$ and frozen in $1 \mathrm{ml}$ aliquots until use. DL-Propranolol Hydrochloride (Sigma) was brought into a solution of 1 $\mathrm{mg} / \mathrm{ml}$ with sterile water, $1 \mathrm{ml}$ aliquots were placed in eppendorf tubes and frozen until use. R-(+) Propranolol Hydrochloride (Sigma) was prepared in the same fashion. Atropine Sulfate (American Reagent Laboratories, Inc.; Shirley, NY) was purchased in $1 \mathrm{mg} / \mathrm{ml}$ vials. Phentolamine Hydrochloride and Hexamethonium (Sigma) were prepared on the day of 
use as $1 \mathrm{mg} / \mathrm{ml}$ and $40 \mathrm{mg} / \mathrm{ml}$ solutions respectively. Isoproterenol (Sigma) was also prepared fresh each day in various concentrations and was shielded from light until used. Reserpine (Sigma; Louis, MO) was compounded $(2.5 \mathrm{mg} / \mathrm{ml})$ with benzyl alcohol, citric acid, Tween 80 and water (Leyden et. al., 1956). This solution was then diluted with sterile water to $1 \mathrm{mg} / \mathrm{ml}$ for intraperitoneal injection. Bromoacetyl Alprenolol Menthane (BAAM) was purchased from Research Biochemicals International (Natick, MA) and was brought into solution with $95 \%$ ethanol and then diluted with $0.9 \%$ normal saline to a final concentration of $40 \mathrm{mg} / \mathrm{kg}$. Normal Saline (Bacteriostatic 0.9\% Sodium Chloride; Abbott Laboratories; North Chicago, IL) was purchased in $30 \mathrm{ml}$ vials. Heparin Sodium Injection (1000 IU/ml; Elkins-Sinn, Inc; Cherry Hill, NJ) was purchased in $10 \mathrm{ml}$ vials.

\section{Buffer}

Krebs-Henseleit (KH) buffer was prepared daily in 2 liter volumetric flasks. Double distilled, nanopure water was used to bring the salts into solution. The final concentration of the reagents, as final concentrations of the salts, was as follows: $\mathrm{NaCl}, 118 \mathrm{mM} ; \mathrm{NaHCO}_{3}$, $25 \mathrm{mM} ; \mathrm{MgSO}_{4}, 1.21 \mathrm{mM}$; Dextrose $10.98 \mathrm{mM} ; \mathrm{KCl}, 4.69 \mathrm{mM}$ and $\mathrm{KH}_{2} \mathrm{PO}_{4}, 1.18 \mathrm{mM}$. The solution was bubbled and stirred for 20 minutes. At this time calcium chloride was added for a final concentration of $\mathrm{CaCl}_{2} \bullet \mathrm{H}_{2} \mathrm{O}, 2.51 \mathrm{mM}$.

\section{In vivo cardiovascular preparation}

Strict sterile technique was observed for all procedures in which the animal recovered. After adequate anesthesia was obtained with Telazol the animals were shaved in the groin and nape of the neck. The animals was then placed supine on a styrofoam cooler lid which had been cleaned with alcohol and draped in a sterile fashion. Rubber bands were placed around each foot and the rubber bands were pinned to the styrofoam to obtain access 
to the groin. The groin was then scrubbed with betadine. A small incision was made in the left groin. Careful dissection was carried out until the femoral vein and artery had been individually isolated. Using 3-0 silk suture proximal and distal control was obtained of each vessel. Catheters had been prepared from 0.010 in. internal diameter X 0.030 in. external diameter Tygon Micro-Bore Tubing, Formulation S-54-HL, Norton Performance Plastics (VWR Scientific Products, So. Plainfield, NJ) approximately 18 inches in length that had been individually sterilized by gas sterilization with ethylene oxide. Each catheter had two "dumbbells". These dumbbells were short pieces of PE-60 tubing that had been heat flared at each end and placed onto the catheter. A small venotomy was made and the catheter was placed, by estimation of the appropriate length, just into the vena cava. Placement and patency was confirmed by aspirating blood and flushing easily with saline and the catheter was clamped with a hemostat covered with plastic tubing so as not to crimp the catheter. The suture used for distal control was then tied gently around the vein and catheter. The suture used for proximal control was tied around a dumbbell immediately distal to the venotomy. In the same fashion an arteriotomy was performed and a catheter inserted. The catheter was placed in the abdominal aorta below the renal artery. Correct placement of the arterial catheter was confirmed by the pulsatile flow of blood through the catheter. If the flow was nonpulsatile the catheter was repositioned. This catheter was tied in place in the same fashion as the venous catheter. The groin incision was then closed with a running vicryl suture with only a small portion of the wound left open. The legs were freed. A hollow steel rod with a beveled sharp end was tunneled subcutaneously from the groin to the nape of the neck where it was brought through the skin. The internal diameter of this "tunneler" was such that a catheter could just pass through. Individually, each catheter was advanced to the 
nape of the neck, the tunneler was removed and the catheters pulled taught. The purpose of the second dumbbell is to create a gentle turn when the catheter is pulled from the groin to the neck. The remainder of the groin wound was then closed. An ultraseude vest was then fitted to the animal. The vests contain a metal hub located over the nape of the neck. The catheters were placed through the hub and then through a spring approximately one foot in length. In this way, the animal could not access the catheter. A 25 gauge hypodermic needle was scored and the sharp end broken off. This needle was then placed into the end of the catheter. Each catheter held approximately $0.1 \mathrm{ml}$ of volume. The catheters were flushed with $0.1 \mathrm{ml}$ of heparin $1000 \mathrm{IU} / \mathrm{ml}$ and capped. The animals were then placed in individual Plexiglas and stainless steel footshock chambers (model 80000, Lafayette Instrument Co., Lafayette, Ind.) modified to provide food and water ad libitum. The spring and catheter exited through the top of the cage so that animals could be hemodynamically monitored and receive injections without being disturbed (Johnson and Kotchen, 1990). After a 24 hour recovery period the catheters were aspirated and then flushed with heparin diluted to 100 $\mathrm{IU} / \mathrm{ml}$, approximately $0.3 \mathrm{ml}$ was used. The catheters were then attached to PE tubing that had been primed with the same dilute heparin solution. In line at the site of attachment of the catheter to the PE tubing was a small animal cannula swivel (375 Series-Single Channel; Instech Laboratories, Plymouth Meeting, PA) that allowed the catheter coming from the animal to rotate freely. The catheter was attached to a Novatrans II pressure transducer (Medex Inc, Hillard, OH). This transducer was then connected through an ETH 400 bridge amplifier (CB Sciences; Dover, HH), and then to a MacLab 8s (ADInstruments; Milford, MA) digital recording system. Data were collected digitally at one kilosecond intervals on a 
MacIntosh LC 550 (Apple Computer; Cuppertino, CA). Blood pressure and HR were determined from the digital tracings using Chart software (ADInstruments; Milford, MA).

\section{Intrinsic heart rate}

Determination of the intrinsic heart rate (IHR) was according to the method of Jose as extended by Cavero (Jose, 1966; Cavero, et. al., 1976) and the design of this experiment is shown in the preceding section. These animals were pretreated with LPS or saline 16-20 hours prior to determination of IHR. Animals were then anesthetized with Nembutal and electrodes attached to their right arm, right leg and left leg. The femoral vein was cannulated in the manner above. The starting HR was recorded as HRN. Propranolol $(8 \mathrm{mg} / \mathrm{kg})$ and atropine $(1 \mathrm{mg} / \mathrm{kg})$ were administered intravenously as described in the experimental design. The heart rate 10 minutes after propranolol is the HRP and the heart rate 10 minutes after atropine is the HRA. The heart rate following both atropine and propranolol equals the HRAP, or HRo, which is the IHR. Cavero described this model using the following calculations:

$$
\begin{aligned}
& \mathrm{HRA}=\mathrm{HRo}_{0} \cdot \mathrm{S} \\
& \mathrm{HRP}=\mathrm{HRo}_{0} \cdot \mathrm{V} \\
& \mathrm{HRAP}=\mathrm{HRo} \\
& \mathrm{HRN}=\mathrm{HRo} \cdot \mathrm{S} \cdot \mathrm{V} \cdot \mathrm{W}
\end{aligned}
$$

Where $\mathrm{S}$ = sympathetic contribution to HRN, V = parasympathetic contribution to HRN and $\mathrm{W}$ equals the interaction, if any between the parasympathetic and sympathetic influences.

In those experiments where hexamethonium was used it was administered in the same fashion as atropine and propranolol. 


\section{In vitro cardiovascular preparation}

For the isolated working left heart preparation (figure 5) the animals were anesthetized with either Nembutal or Telazol as described above. The abdomen was then opened and a bilateral thoracotomy quickly performed. With gentle lifting of the heart the great vessels were cut with scissors and the heart immediately placed into ice-cold buffer where it arrested. The Langendorff apparatus had at this point been warmed to $37.5^{\circ} \mathrm{C}$ via jacketed glassware. With the buffer running the aortic root was mounted on the aortic cannula well above the aortic valve and tied in place. The heart was perfused with warm physiological salt solution via the Langendorff column at $85 \mathrm{~cm} \mathrm{H}_{2} \mathrm{O}$ pressure and began to beat. The total ischemic period was less than one minute. The insertion point for one of the pulmonary veins was identified in the left atrium. The atrial cannula was inserted into this opening and tied with a ligature around the atrium and cannula. Care was taken to not incorporate the right atrium. The pulmonary artery was incised to insure decompression of the right ventricle. At this point the apparatus was converted to a working left heart preparation by closing the Langendorff column and opening the atrial reservoir. The preload on the heart was set at $15 \mathrm{~cm} \mathrm{H}_{2} \mathrm{O}$ by adjusting the height of the atrial reservoir above the left atrium, the afterload was set at $70 \mathrm{~cm} \mathrm{H}_{2} \mathrm{O}$ by adjusting the height of the aortic outflow tubing. The buffer was continually oxygenated with a mixture of $95 \%$ oxygen and $5 \%$ carbon dioxide and recirculated. A filter prevented the recirculation of anything large enough to occlude the coronary arteries. The apparatus was thoroughly rinsed and the filter changed between each heart. The aortic cannula has an opening through which pressure tubing connects it a transducer (Novatrans II pressure transducer; MedexInc, Hillard, OH). The pressure tracing and beating rate were recorded on an intensive care monitor (Spacelab 
90623A; Redmond, WA). Cardiac output was recorded by collecting the aortic flow (AF) in a burrel pipette and the coronary flow (CF) measured as it drips off the heart. Together AF and $\mathrm{CF}$ determine CO. Heart rate is recorded by counting the aortic deflections. Stroke volume is derived by dividing $\mathrm{CO}$ by HR. After a 20 minute stabilization period, from the time of placement in the working mode, was observed. Measurements were obtained at 5 minute intervals.

Figure 5. Isolated left heart perfusion apparatus. The heart sits in the buffer cannulated at the aorta and left atrium. Buffer is ejected through the damping chamber and aortic flow tubing (afterload). It is collected as aortic outflow. The buffer is oxygenated in the atrial reservoir the height of which determines preload. To determine afterload the bath is lowered and the effluent from the heart is collected. To perfuse the heart as a Langendorff preparation the Langendorff column is opened and the atrial tubing closed off. (Diagram used and modified with permission of $R C$ Hill).

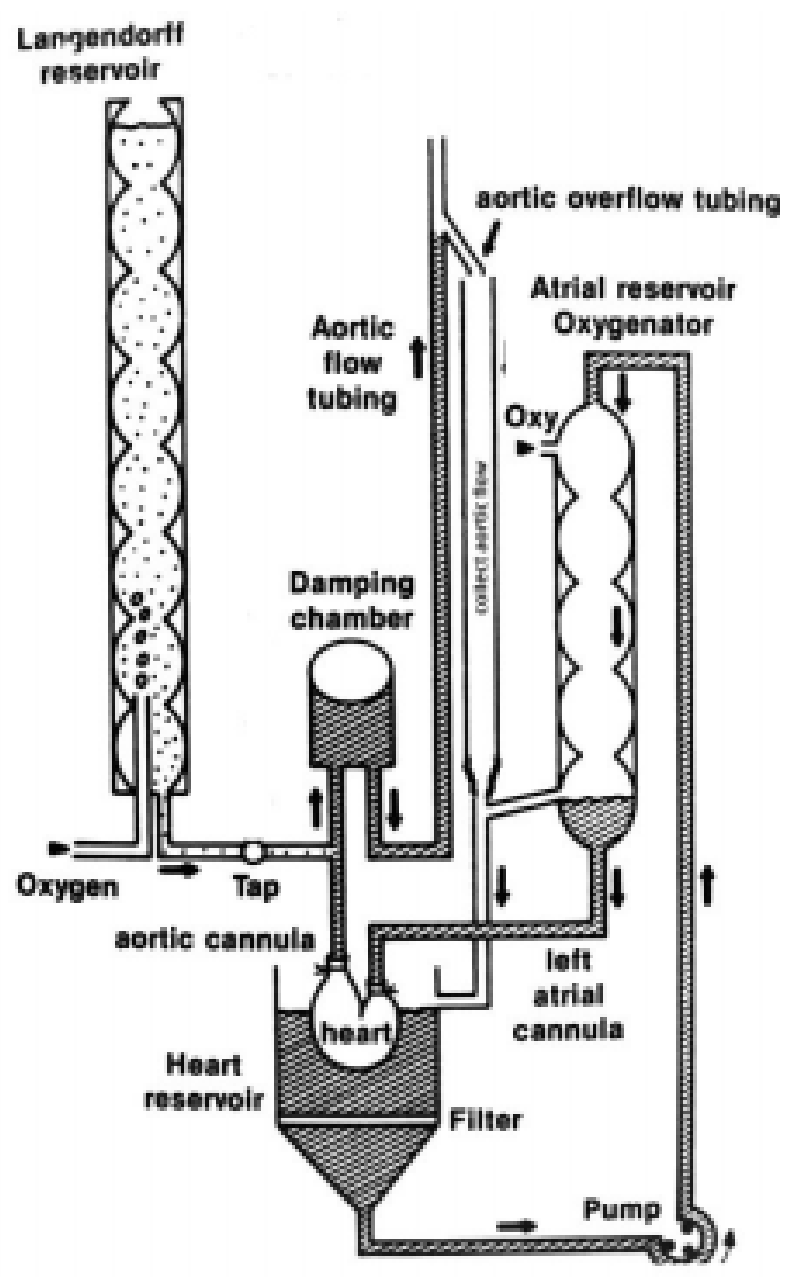

In the experiments where the rate of isometric tension generation was determined (i.e.

V dP/dt max and min), the method of Apstein (Apstein et. al., 1977) was used. Briefly this 
method is as follows: The heart was again placed into the Langendorff perfusion mode and the left atrium removed from the atrial cannula. Via the opening in the left atrium, a latex balloon tipped catheter was inserted through the mitral valve into the left ventricle. This catheter was attached to the pressure transducer which, in this experiment, was connected to the MacLab apparatus. By adjusting the amount of volume (saline) in the balloon, preload or left ventricular end-diastolic pressure, could be established. In these experiments the heart was paced with a stimulator (Grass S48; Astromed; W. Warwick, RI) via an electrode placed on the right atrium. The minimum voltage necessary to capture the heart was used, generally 1-3 Volts. The left ventricular developed pressure (LVDP) was determined as the maximum systolic pressure. The Chart software calculates the first derivative of the pressure tracing in order to determine $\mathrm{V}$ Dp/dt max.

In those experiments in which drug was added to the isolated heart preparation it was added directly to the buffer bath. The recirculation time was noted to be approximately 5 minutes. All measurements were carefully recorded at the exact time from the addition of the drug. In all experiments, except the isoproterenol concentration response curve and the propranolol concentration response curves, only one dose of drug was administered in vitro. The concentration response curves for isoproterenol and propranolol were cumulative.

\section{Isolated Right Atria Preparation}

In the experiment that utilized the isolated right atrial preparation the animals were anesthetized, the hearts extirpated and arrested in a manner identical to that described for the in vitro cardiovascular preparation described above. After the excised heart was arrested the right atrium was dissected free from the remainder of the heart. Care was taken to avoid crushing or excessive handling of the atrium. Once it had been dissected free 5-0 silk sutures 
were gently tied to opposite ends of the atrium. The atria were then placed in water jacketed organ baths containing $\mathrm{KH}$ buffer bubbled with $95 \%$ oxygen $5 \%$ carbon dioxide maintained at $37^{\circ}$. The suture was attached to a Grass FT .03 force-displacement transducer.

Contractions of the spontaneously beating right atrial preparations were passed via a Grass polygraph to the MacLab where the tracings were digitized. The heart rates were calculated off line utilizing the Chart software. All atria were provided with at least a 40 minute stabilization period. Four atria, determined at random, could be examined simultaneously via separate organ baths. The isoproterenol dose response curves were begun after the recording of the basal heart rates (5 minutes after the stabilization period). The cumulative concentration response curve was observed for single log units by adding $10 \mu \mathrm{l}$ of appropriate concentrations of isoproterenol $\mathrm{HCl}$ to the $10 \mathrm{ml}$ organ bath to bring the final concentration from $10^{-16}$ to $10^{-5}$. The HR was determined four minutes after the addition of each successive dose. Four minutes after the completion of the dose response curve the water bath was rinsed four times by adding $10 \mathrm{ml}$ of fresh $\mathrm{KH}$ buffer and then immediately draining it. The HR was recorded every 5 minutes for one hour after this wash. Every 15 minutes within this time period the baths were again rinsed with fresh KH buffer.

\section{Western Blots}

The heart were extirpated in a fashion identical to that described above, placed on the Langendorff perfusion apparatus and perfused with warm KH buffer for approximately two minutes. This was done to flush the heart of any blood. The right atrium was then excised, placed in tinfoil and snap frozen in liquid nitrogen. All specimens were stored at $-80^{\circ}$ for further analysis. On the day of protein isolation the specimens were quickly weighed and placed in $1.0 \mathrm{ml}$ of protease inhibitor buffer (PIB) per $100 \mathrm{mg}$ of tissue. The PIB consisted 
of $0.25 \mathrm{M}$ sucrose, $1.0 \mathrm{mM}$ EDTA, $4.0 \mathrm{mM}$ phenylmethylsulfonyl flouride, $1.0 \mathrm{mM}$ aminobenzamidine and $1 \mathrm{mg} / \mathrm{ml}$ of bacitracin. At this point tissues were homogenized by hand utilizing a Duall tissue grinder. The homogenate was microcentrifuged for 5 seconds to remove nonhomogenized tissue. The supernatant was transferred to fresh Eppendorf tubes and refrozen as individual aliquots to avoid repeated freeze-thawings. On the day of western blotting the tubes were thawed and microcentrifuged at $14,000 \mathrm{rpm}$ for 15 minutes. A single aliquot of the supernatant was used for protein determination by the method of Bradford (Bradford, 1976). Based upon this determination each sample was diluted in Laemmli sample buffer (Amersham Life Science; Piscatway, NJ) to equal dilutions. Five $\mu$ of specimen and sample buffer containing $4 \mu \mathrm{g}$ of protein were then loaded into wells of a 15 well 10\% Tris-HCl minigel (Biorad Laboratories; Hercules, CA). Electrophoresis was performed at constant voltage $(115 \mathrm{mV})$ in Tris/glycine/SDS running buffer (Biorad Laboratories; Hercules, CA) for approximately one hour. The separated proteins were immediately transferred to polyvinylidene diflouride sequencing membrane (Biorad Laboratories; Hercules, CA) using a HEP-1 semi-dry transfer apparatus (OWL Scientific Plastics) using a constant current of $200 \mathrm{~mA}$ for one hour. Transfer was carried out between two gel blotting papers (Schleicher \& Schuell; Keene, NH) soaked in transfer buffer. The transfer buffer consisted of $39 \mathrm{mM}$ glycine, $48 \mathrm{mM}$ Tris base, $0.037 \%$ SDS and 200ml of methanol brought to one liter and a $\mathrm{pH}$ of 8.3. After transfer the membranes were allowed to dry completely and then washed with phosphate buffered saline containing $0.1 \%$ Tween-20 (PBST). Following this the membranes were blocked for one hour with $3 \%$ dry milk in PBST. The membranes were again washed and the primary antibody (mouse anti-PKA-C; Transduction Laboratories; Lexington, KY) at a concentration of 1/1000 with 3\% bovine 
serum albumin (BSA) in PBST was applied for one hour. The membranes were again washed and a goat-anti-mouse-HRP conjugated secondary antibody (Biorad Laboratories; Hercules, CA) was applied for one hour at a concentration of 1/3000. At the completion of the secondary antibody exposure the membrane was washed and protein detection was carried out with an enhanced chemiluminesence system (ECL, Amersham Life Science; Piscataway, NJ) and developed on Hyperfilm ECL film (Amersham Life Science.)

Quantification of protein was determined by the densitometric analysis of the western blots. Briefly the blots were place on a light source (Precision illumination model B90, Imaging Research Inc.; St. Catherines, Ont.) and captured with a Dage CCD 72 camera (Dage; Michigan City, IN). Boxes were hand drawn with around each blot utilizing a PC and mouse. The inverse log of the integrated gray value (the mean gray value $\mathrm{x}$ area) was determined with Optimas imaging software (Optimas Corp.; Edmunds, WA). Two control animals and two animals for each treatment group were present on each blot in order to control for variability in the processing. The experiment was duplicated at separate time points. The final analysis was therefore conducted on three separate protein aliquots for four animals in each treatment group. All densitometric units were normalized to the average of the two controls on each blot.

\section{Heart Rate Variability}

The heart rate variability studies were performed on electrocardiogram (ECG) tracings from conscious rats. Rats were anesthetized with Telazol and subcutaneous wires (.010 in. PVC coated wire, Belden; Geneva, IL) were sutured to either side of the chest wall. The wires were tunneled subcutaneously to the nape of the neck and brought out through the vest, hub and spring combination utilized in the in vivo cardiovascular 
preparation. The animals recovered in the plexiglass foot shock chamber for 24 hours prior to the beginning of the experiment. In four animals a control ECG tracing was then recorded continuously for 24 hours by attaching the electrodes to the SpaceLab intensive care unit monitor and recording ECG lead I. The analog ECG signal was passed from the SpaceLab monitor to the MacLab where the tracing was sampled at 400 samples per second, digitized and recorded on the hard drive. After 24 hours the animals were injected with LPS $5 \mathrm{mg} / \mathrm{k}$ i.p. and their ECG tracing recorded for an additional 16 hours. The heart rate variability (HRV) extension of the Chart software was programmed to take equally spaced 5 minute samples from each of the 40 hours for time and frequency domain analysis. The other five animals were handled identically with the exception that ECG was not recorded continuously but instead five minute recordings were taken at 24, 20, 16, 12, 8, 4 and 1 hour before LPS administration and every hour for eight hours and then 12 and 16 hours after LPS.

All of the five-minute samples were examined for a stationary baseline or significant artifact. If this existed the sample was rejected and another five-minute tracing was obtained. All samples were processed utilizing the Chart software in accordance with the "Heart rate variability: standards of measurement, physiological interpretation, and clinical use" from the Task Force of the European Society of Cardiology and the North American society of Pacing and Electrophysiology" (Malik, 1996). Detection of the R wave was performed on the derivative of the ECG tracing to eliminate respiratory artifact. The Chart program allows for the manual setting of the threshold of $\mathrm{R}$ wave detection with a precise fiducial point. All RR intervals between 100 and $200 \mathrm{~ms}$ were included, ectopics were excluded and artifacts manually edited. Measurements calculated in the time domain included the standard deviation of all normal RR intervals (SDNN) and the root mean of the sum of squares of 
differences between adjacent RR intervals (RMSDD). The power spectrum was calculated with fast Fourier transformation by automatic resampling of the tachogram generated via the above method. The algorithm included linear interpolation, no overlapping and the use of a Hann window. The power spectrum was calculated on a 512 point segment. The low frequency (LF) was set at $0.1 \mathrm{~Hz}$ to $0.8 \mathrm{~Hz}$ and the high frequency (HF) as 0.8 to $3 \mathrm{~Hz}$. The parameters calculated included the LF and $\mathrm{HF}$ variability, the ratio LF/HF and the normalized units of HF (HF nu) and LF (LF nu). HF nu and LF nu were calculated by dividing HF and LF by total power (measure of total variability) after subtracting the very low frequency variability from the total power.

\section{Statistical Analysis}

The method of statistical analysis was an analysis of variance (ANOVA) except for those instances where Student's t-test or regression analysis were appropriate. In general, the ANOVA model utilized was a modified split plot design with repeated measures. The error term consisted of animals nested within treatments. Treatments (LPS or saline and drugs) and time were handled individually and as factorials in order to test for interactions among the variables. In some experiments heart rate was used as a covariate to control for its effects on contractile function. Multiple comparisons within the ANOVA were made only when the F test indicated it to be appropriate. At this point comparisons were made with orthogonal contrasts. The $\mathrm{p}$ value of the t-test of these contrasts required for significance was determined by dividing the alpha value (0.05) by the number of comparisons made according to the method of Bonferroni (Tukey, 1949; Kramer, 1956). This method of multiple comparisons does not require that $\mathrm{n}$ values for each treatment group be equal. In those instances where one-way ANOVA was performed, the Tukey-Kramer test was used for 
multiple comparisons. All analyses were carried out using JMP statistical software (SAS Institute, Research Triangle Park, NC).

Dose response curves were fitted and graphed with Prism software (GraftPad Software, Inc.; San Diego, CA). The ED50 and EC50 values were calculated as geometric means with 95\% confidence intervals according to the method of Fleming (Fleming et. al., 1972). 


\section{Results}

\section{Characterization of the model}

\section{a. In vivo cardiovascular changes following endotoxin}

By four hours following the administration of endotoxin the animals generally moved less and appeared lethargic. Endotoxin treated animals had stopped grooming behavior and drank less than control animals. Septic animals demonstrated piloerection, somewhat labored breathing and frequently their eyes became encrusted with exudates. These findings were progressive over time. The mortality rate following the bolus endotoxin injection was approximately eight percent and, for the most part, occurred within the first 12 hours. In examining 204 of the rats used in these experiments the initial weights were not significantly different, however there was a $4.8 \%$ average weight loss in those animals treated with endotoxin which was significantly different from a gain of $0.6 \%$ that was seen in the control animals (table 1).

\begin{tabular}{|c|c|c|c|}
\hline & Pre Weight (grams) & Post Weight (grams) & \% Change in Weight \\
\hline Control & $410 \pm 4.6$ & $412.3 \pm 4.5$ & $+0.6 \pm 0.2$ \\
\hline LPS & $418 \pm 3.8$ & $398.4 \pm 3.7$ & $-4.8 \pm 0.1$ \\
\hline & $\mathrm{p}=0.1592$ & $\mathrm{p}=0.0178$ & $\mathrm{p}<0.0001$ \\
\hline
\end{tabular}

Table 1. Demonstrates the starting weight (pre-weight), weight at the time of sacrifice (post-weight) and \% change in weight for control and endotoxin (LPS) treated animals 16-20 hours after LPS. These data are from 204 animals that received only saline or LPS pretreatment. The $R^{2}$ for $\%$ change in weight $=0.64$.

Eight animals underwent the placement of left femoral artery catheters for the purpose of studying the time course of blood pressure and in vivo heart rate changes in the twenty four hours following $5 \mathrm{mg} / \mathrm{kg}$ endotoxin administration. This model of endotoxemia demonstrated no significant changes in blood pressure for the full 24 hours and in vivo HR 
was not significantly elevated until fours after the i.p. dose of LPS after which it remained elevated for the remainder of the time monitored (figure 6).

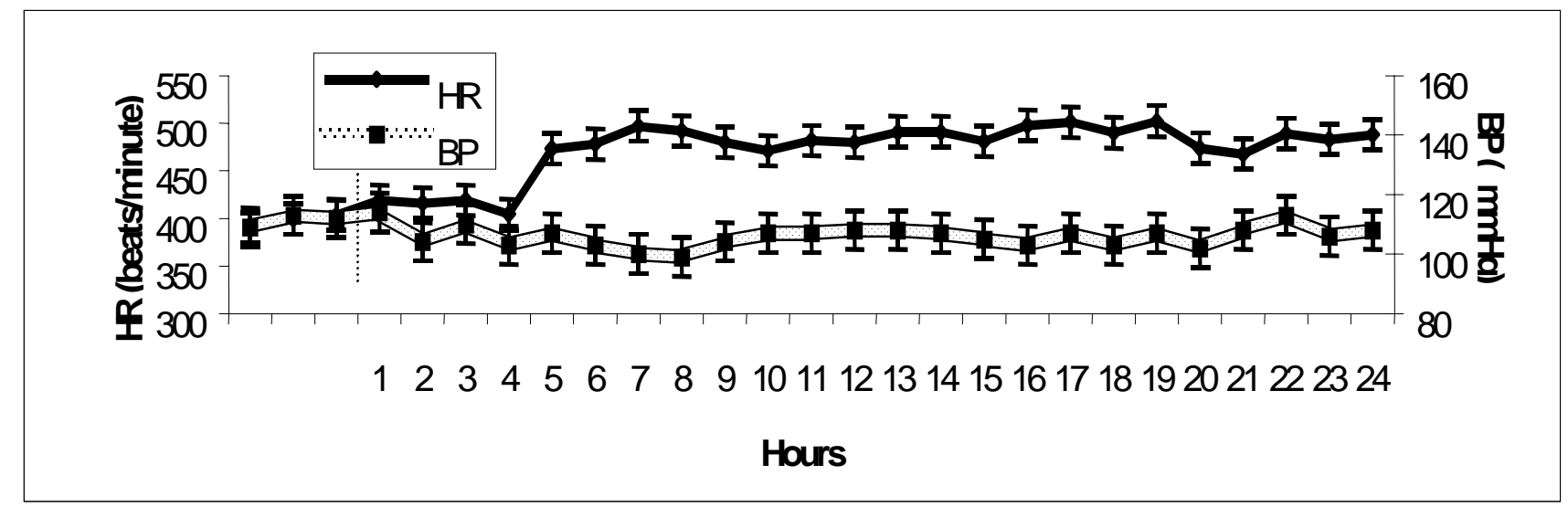

Figure 6. In vivo changes in heart rate $(H R)$ and blood pressure $(B P)$ for 3 hours before and 24 hours following LPS administration (dotted vertical line). Blood pressure is not significantly altered throughout the time course. HR becomes elevated at 5 hours and remains elevated through 24 hours ( $p<0.05)$. Error bars represent \pm SEM.

It should be noted that this tachycardia is a sinus tachycardia based upon ECG tracings. In electrocardiogram tracings obtained from septic animals there was a constant $\mathrm{P}$ wave and no alterations in the P-R interval were found while the P-P interval was significantly reduced (data not shown).

\section{b. Determination of intrinsic heart rate}

The finding of an in vivo tachycardia following endotoxin administration was anticipated. The next experiments were conducted to determine if any of the increase in HR was related to an elevation of the intrinsic heart rate (IHR). The first experiment designed to address this issue was the administration of atropine and propranolol to achieve complete autonomic blockade according to the method of Cavero (Cavero et. al., 1976). In reviewing the literature, the previous doses of drugs used for this purpose, in the rat, ranged from 1 $\mathrm{mg} / \mathrm{kg}-10 \mathrm{mg} / \mathrm{kg}$ i.v. for propranolol and $200 \mu \mathrm{g} / \mathrm{kg}-4 \mathrm{mg} / \mathrm{kg}$ i.v. for atropine (Ackerman et. al., 1987; Horowitz and Meiri, 1993; Machado and Brody, 1989; Machado et. al., 1987; 
Muntzel and Peterson, 1997; Soato and Krieger, 1974). We conducted preliminary

experiments in which doses above $8 \mathrm{mg} / \mathrm{kg}$ of propranolol resulted in no additional slowing of the heart rate and doses of atropine above $1 \mathrm{mg} / \mathrm{kg}$ yielded no further increase in HR.

These doses were consistent with previous studies in the literature and were employed in the experimental paradigm.

Figure 7 demonstrates the change in HR following the administration of atropine (1 $\mathrm{mg} / \mathrm{kg}$ ). There was little parasympathetic tone in the anesthetized animal as evidenced by the very slight increase in HR with atropine (figure 7).

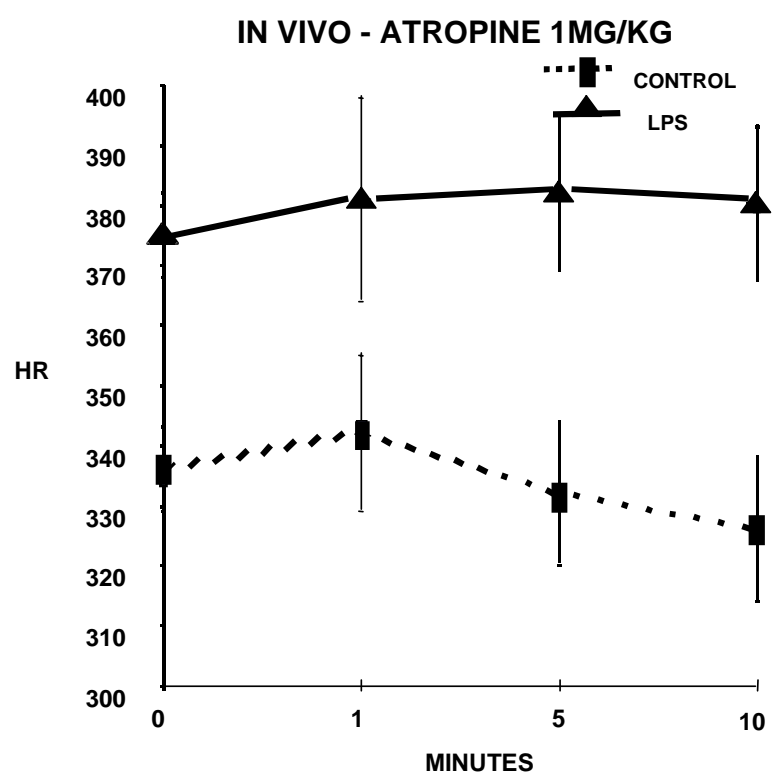

Figure 7. Demonstrates the change in HR following the administration of atropine (1 $\mathrm{mg} / \mathrm{kg})$ to anesthetized rats in vivo 16-20 hours after LPS $(5 \mathrm{mg} / \mathrm{kg})$ or saline. Atropine was administered between 0 and 1 minute. HR remained significantly different $p<0.05$ at all time points. Error bars represent \pm SEM.

An equal number of rats were administered propranolol first, these results are shown below (figure 8). Those animals administered propranolol clearly demonstrate that the major autonomic tone in the anesthetized rat is sympathetic. The effect on HR following 
propranolol treatment remained parallel, however, suggesting that the increase in HR in LPS treated animals is not due to sympathetic influences.

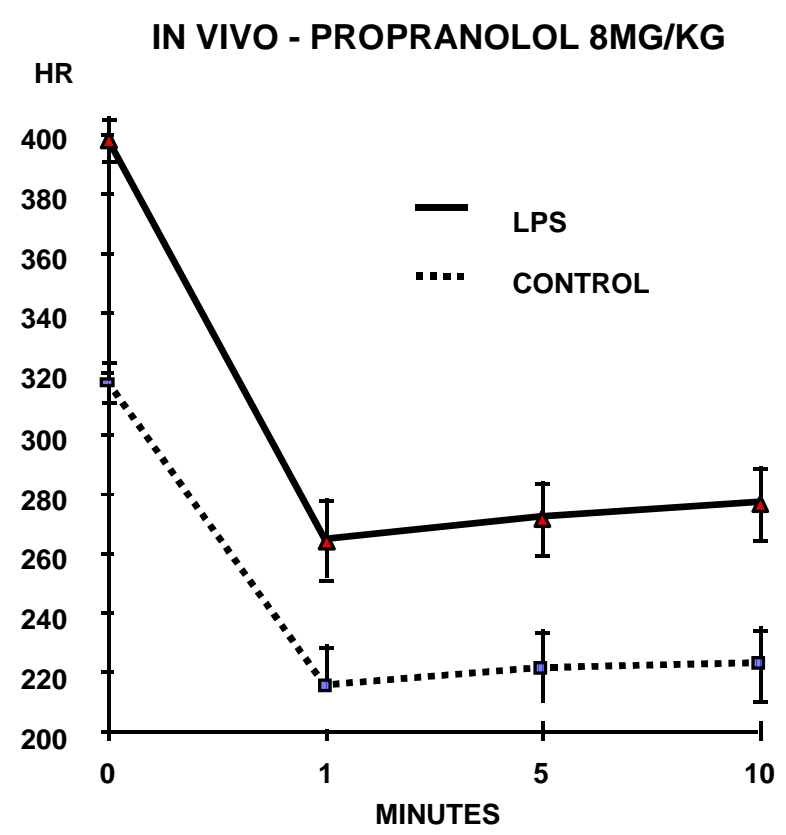

Figure 8. Demonstrates the change in HR following the administration of propranolol (8 $\mathrm{mg} / \mathrm{kg})$ to anesthetized rats in vivo 16-20 hours after LPS $(5 \mathrm{mg} / \mathrm{kg})$ or saline. Atropine was administered between 0 and 1 minute. HR remained significantly different $p<0.05$ at all time points. Error bars represent \pm SEM.

At 10 minutes after the administration of atropine or propranolol all animals then received the second agent, resulting in blockade of both components of the autonomic nervous system and revealing the intrinsic heart rate. Ten minutes after administration of the second agent the intrinsic heart rate or $\mathrm{HR}_{\mathrm{o}}$ was recorded. The initial heart rate before the administration of any drug was $19 \%$ greater in endotoxin treated animals. After the administration of both atropine and propranolol there was a $21 \%$ difference in the HRo. Interestingly, however, there was also no difference in the sympathetic and parasympathetic inputs to the in vivo 
heart rate (Table 2). These data suggested that all of the difference in heart rate, as a

percentage change caused by endotoxin was due entirely to changes in the intrinsic heart rate.

\begin{tabular}{|l|l|l|l|l|}
\hline TREATMENT & HRn & HRo & $\% \mathrm{~S}$ & $\% \mathrm{~V}$ \\
\hline Control & $327 \pm 11$ & $246 \pm 6$ & $39 \pm 4 \%$ & $-14 \pm 2 \%$ \\
\hline LPS & $390 \pm 11$ & $299 \pm 6$ & $35 \pm 5 \%$ & $-12 \pm 2 \%$ \\
\hline & $\mathrm{p}=0.0005$ & $\mathrm{p}<0.001$ & $\mathrm{p}=0.50$ & $\mathrm{p}=0.069$ \\
\hline
\end{tabular}

Table 2. Relative contributions to HR via the sympathetic and parasympathetic nervous systems. HRn = initial HR. HRo = intrinsic heart rate. $\% S=$ sympathetic contribution to $H R$. \% V = parasympathetic contribution to HR. HR's are represented as beats per minute (bpm \pm SEM). $n=12$ rats/group

Autonomic transmission at both the sympathetic and parasympathetic ganglia is via acetylcholine binding at nicotinic receptors. Antagonism of these receptors with hexamethonium $(40 \mathrm{mg} / \mathrm{kg})$ should duplicate the surgical excision of the heart at the level of the ganglia by inhibiting autonomic input to the heart. In a separate set of animals exposed to endotoxin or saline 16-20 hours earlier, hexamethonium did not abolish the difference in HR. The heart rate following endotoxin treatment was $23 \%$ higher than the HR of control animals (table 3).

\begin{tabular}{|c|c|c|}
\hline TREATMENT & HRn & HRhex \\
\hline Control & $358 \pm 11$ & $272 \pm 9$ \\
\hline LPS & $409 \pm 10^{*}$ & $334 \pm 10^{*}$ \\
\hline
\end{tabular}

Table 3. In vivo HR before and after the administration of hexamethonium $(40 \mathrm{mg} / \mathrm{kg}$ i.v.) to anesthetized rats. HRn = initial HR, HRhex $=H R 15$ minutes following hexamethonium treatment. LPS (5 $\mathrm{mg} / \mathrm{kg}$ was administered 16-20 hours prior to anesthesia and recording of HR. HR (bpm \pm SEM). * = different from control $p<0.01$. $n=7$ per group

This experiment confirms the earlier findings of an elevation of intrinsic heart rate, although it does not exclude the possibility of circulating catecholamines (epinephrine) effecting the heart rate. Neither method of pharmacologic denervation removes the heart from the presence of humoral substances, other than catecholamines, which could influence the HR. Excision of the heart, and perfusion with oxygenated buffer as an 
isolated working heart, does provide an experimental means to abolish both neural and humoral influences on the heart.

\section{c. Determination of in vitro heart rate}

Animals were again treated with LPS or saline 16- 20 hours prior to the administration of anesthetic. After adequate anesthesia was obtained, the hearts were extirpated and perfused as working left heart preparations and allowed to beat at their intrinsic rate. An equilibration period of 20 minutes was observed prior to the study of rate. In examining 153 rats (76 control, 77 LPS) that had no treatment other than LPS or saline before excision of the heart and recording of the in vitro HR, the LPS treated animals had a $17 \%$ higher rate (Table 4).

\begin{tabular}{|c|c|}
\hline & HR (bpm) \\
\hline CONTROL & $238 \pm 3.6$ \\
\hline LPS & $278 \pm 3.6^{*}$ \\
\hline
\end{tabular}

Table 4. Heart rate (HR) in beats per minute 16-20 hours following endotoxin administration $(5 \mathrm{mg} / \mathrm{kg})$. HR shown as bpm \pm SEM. HR values represent one reading at 20 minutes after isolation of the heart. $*=$ significantly different from control ( $p<$ $0.0001)$

For sixteen rats the in vitro HR was followed for 40 minutes after the initial stabilization period, or for one hour total after excision. In those animals treated with LPS the HR was $267 \pm 7 \mathrm{bpm}$ compared to a rate of $237 \pm 6 \mathrm{bpm}$ for the control animals $(p=0.002)$. As can be seen in figure 9, there is no effect of time (i.e. the HR line is linear, $\mathrm{p}=0.99$ ) after the stabilization period and the in vitro HR remained elevated for at least one hour following extirpation (figure 9). 


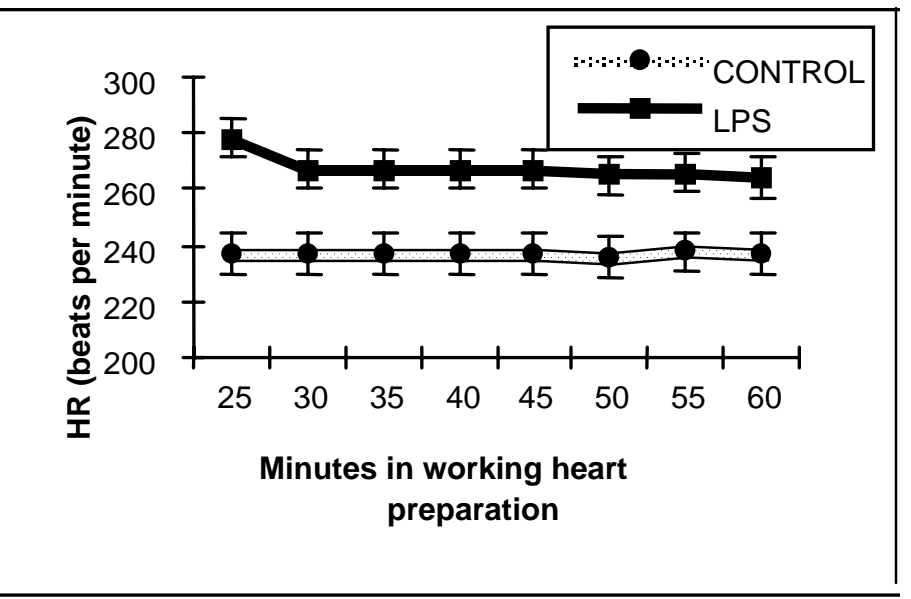

Figure 9. Demonstrates the in vitro HR following a single dose of LPS 16 hours prior. LPS HR is significantly greater than control. $(p=0.0002, n=7$ per group). Error bars represent \pm SEM. The two lines are parallel to each other $(p=0.99)$

In the course of these experiments two variables were identified, other than the experimental variable, LPS treatment, which effected HR. These variables were weight and anesthesia. The effect of basal weight on the in vitro HR was examined from the data of 152 animals. Regression analysis showed a decrease of 0.2 beats per minute for each gram increase in body weight. The $p$ value for this regression line was $p=0.0176$ however the $\mathrm{R}^{2}$ was only 0.037 . This suggests that while weight is a significantly related to the in vitro $\mathrm{HR}$ in our experiments it only explains $3.7 \%$ of the total variability in $\mathrm{HR}$. It should be noted that in none of the experiments was the basal weight significantly different between treatment groups. Because of this lack of difference in initial weights, and because of the small predictive value of weight, this parameter was not included in any of the statistical models. As was noted in the methods, the anesthetic used was changed from pentobarbital to Telazol during the course of these experiments. To examine the effect of anesthetic on in vitro HR an analysis was performed on 78 rats having either pentobarbital or Telazol as their anesthetic. The heart rate following pentobarbital was $237 \pm 7$ compared to $265 \pm 8$ for Telazol. This difference of 28 beats 
per minute, or $11.8 \%$ is significantly different $(\mathrm{p}<0.0001)$. However, there was no interaction between anesthetic and treatment in the statistical model $(p=0.73)$, indicating that the anesthetic effect was equal between treatment groups (figure 10).

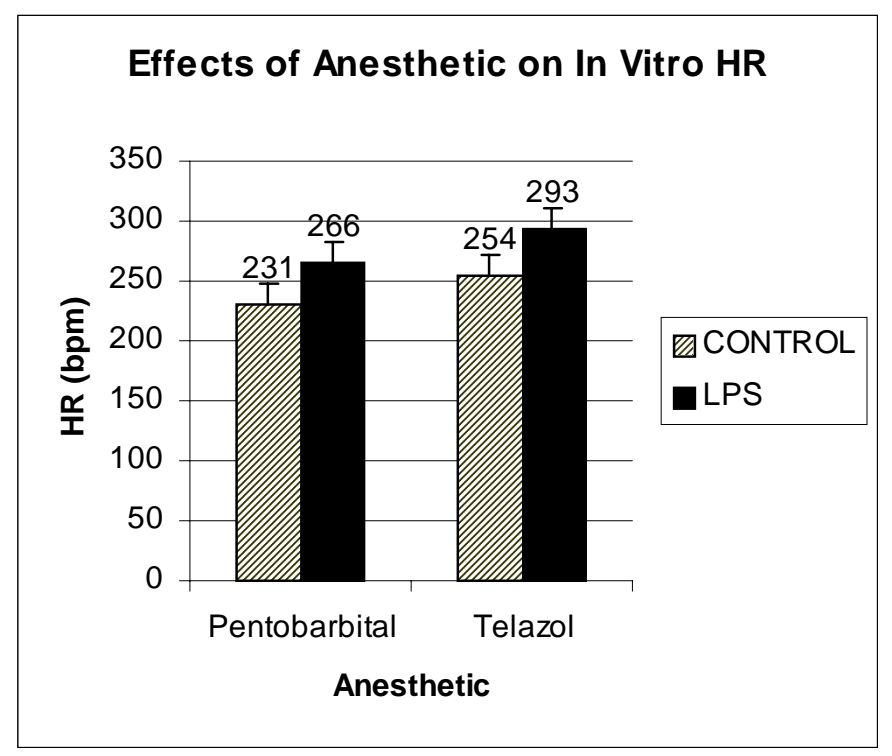

Figure 10. This figure shows the relative effects of anesthetic on in vitro HR. Both anesthetics were administered 16 - 20 hours after LPS (5 $\mathrm{mg} / \mathrm{kg})$ or saline.

Pentobarbital was given at a dose of $40 \mathrm{mg} / \mathrm{kg}$ i.p. and Telazol at30 $\mathrm{mg} / \mathrm{kg}$ i.p. Analysis of variance showed a significant anesthetic effect $(p<0.0001)$ but no interaction between anesthetic and treatment $(p=0.7321) . \quad n=78$

In none of the experiments was more than a single anesthetic used and anesthetic was therefore not included in the statistical models. The possibility existed that the elevation of in vitro HR was due to the unstimulated release of catecholamines while the heart was on the perfusion apparatus. In order to rule out this possibility the nonspecific $\beta$ AR antagonist, propranolol, was added directly to the recirculating buffer at several concentrations. After a 20 minute stabilization period the in vitro HR was again recorded, then propranolol was added to the recirculating buffer to achieve a final concentration of $1 \mu \mathrm{M}$. At this concentration, while there was a significant decrease in HR in both groups, the HR of the LPS treated animal remained significantly greater than 
control. At a concentration of $10 \mu \mathrm{M}$, however, the difference between LPS treated and control animals was abolished (figure 11). This observation and the observation that 1 $\mu \mathrm{M}$ caused a significant decrease in the HR in both groups suggested that there was ongoing stimulation of the $\beta \mathrm{AR}$ in vitro.

\section{In Vitro HR Response to a Racemic Mixture of Propranolol}

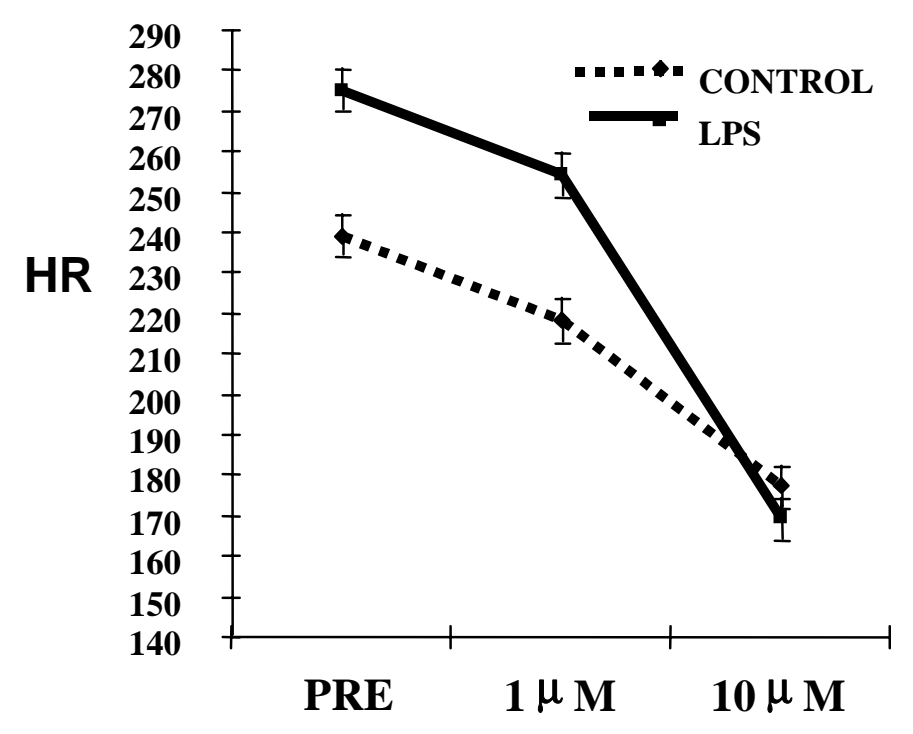

Figure 11. Demonstrates the in vitro dose response curve to increasing doses of RS - propranolol added directly to the bath. LPS animals were treated in vivo with 5 $\mathrm{mg} / \mathrm{kg}$ of E. coli endotoxin 16-20 hours prior. Pre (basal) heart rates (HR) in beats per minute were recorded after a 20 minute stabilization period. Other HRs were recorded 15 minutes after adding the drug. Error bars represent \pm SEM.

Some $\beta$ AR antagonists, including propranolol have non-specific, or membrane stabilizing properties, which are not related to antagonism of the $\beta A R$. These properties could result in a decrease in the HR. It has been shown, however, that this effect 
is observed with both the active and inactive isomers of propranolol (Doggrel, 1990). In order to determine if the decrease in HR that was observed in vitro with racemic propranolol was due to this non-specific effect, concentration response curves to the inactive isomer of propranolol were conducted in the same fashion as above (Figure 12).

\section{In Vitro HR Response to the Inactive Isomer of Propranolol}

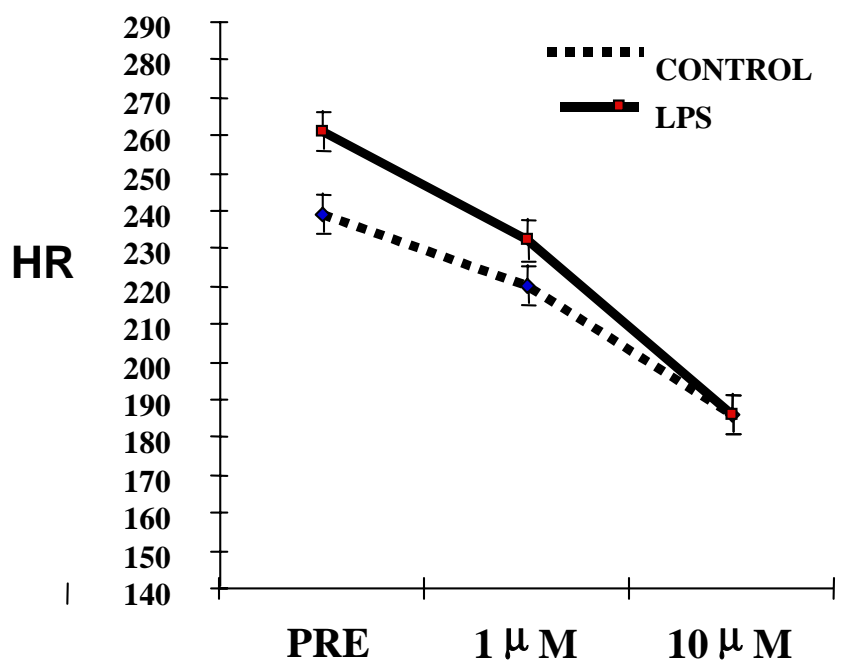

Figure 12. In vitro dose response curve to increasing doses of the inactive $R(+)$ isomer of propranolol added directly to the bath. LPS animals were treated in vivo with 5 $\mathrm{mg} / \mathrm{kg}$ E. coli endotoxin $16-20$ hours prior. Pre (basal) heart rates (HR) in beats per minute were recorded after a 20 minute stabilization period. Other HRs were recorded 15 minutes after adding the drug. Error bars represent \pm SEM.

With the inactive isomer, a concentration of $1 \mu \mathrm{M}$ did abolish the difference in $\mathrm{HR}$ between LPS and control animals. There was a further decline of heart rate with the 10 $\mu \mathrm{M}$ concentration. These data suggested that at the $1 \mu \mathrm{M}$ and $10 \mu \mathrm{M}$ concentrations, the effects observed with the racemic mixture were likely due to actions not related to $\beta \mathrm{AR}$ antagonism and that the elevation of in vitro HR was not due to the presence of catecholamines. It has been suggested that $\alpha$-adrenoceptor stimulation can result in a 
positive chronotropic response (DeLuca and Rand, 1988; Flavahan and McGrath, 1981).

To rule out the unlikely possibility that catecholamines were present and acting at the $\alpha-$ adrenoceptor and that this resulted in the increase in in vitro $\mathrm{HR}$ an experiment was conducted antagonizing both the $\alpha$ ARs and $\beta$ ARs simultaneously. Animals were again treated with LPS or saline, the hearts extirpated and perfused. Exposure of the isolated heart to a concentration of $0.1 \mu \mathrm{M}$ propranolol and $0.1 \mu \mathrm{M}$ phentolamine, a nonspecific $\alpha \mathrm{AR}$ antagonist, did not abolish the LPS induced increase in HR (Figure 13).

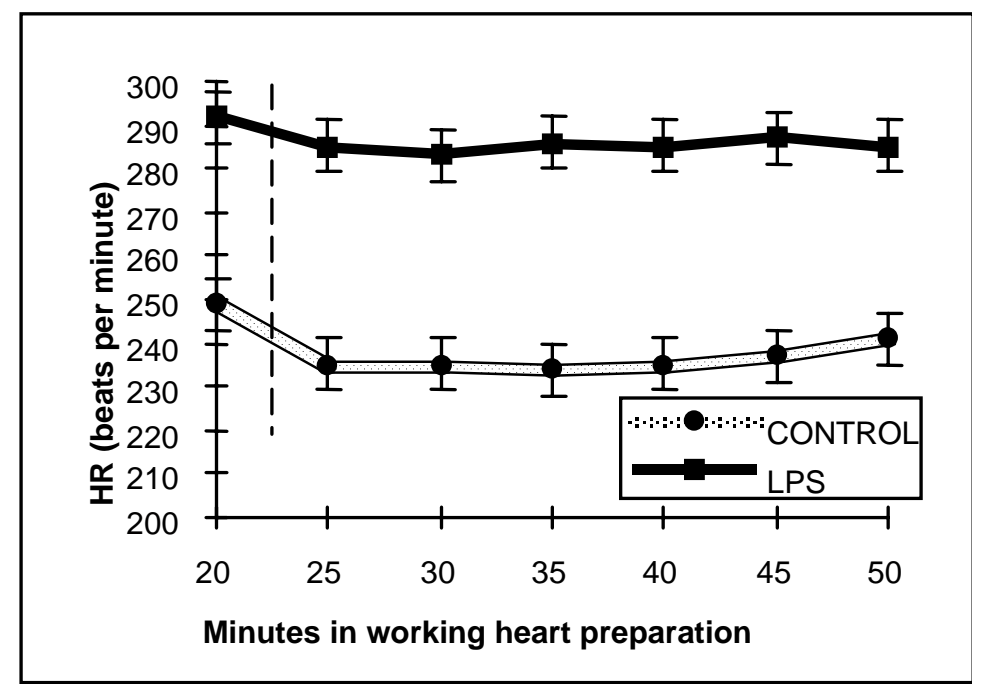

Figure 13. Response of in vitro HR to propranolol $0.1 \mu \mathrm{M}$ and phentolamine $0.1 \mu \mathrm{M}$ added to the bath at the broken vertical line. Animals had been treated in vivo with 5 $\mathrm{mg} / \mathrm{kg}$ endotoxin (LPS) or saline (Control) $16-20$ hours earlier. Error bars represent \pm SEM. LPS line is significantly greater than control $p<0.0001$.

These experiments demonstrated that there was an increase in the in vitro heart rate in addition to the increase in the IHR as measured by pharmacologic denervation.

Together this suggests that there is a resetting of IHR in animals exposed to a single bolus of endotoxin. The reader will recall from the introduction that to date this type of long term change in IHR has only been demonstrated as decreases in IHR observed in 
both exercise training and with age. The goal of the next series of experiments was to explore and determine the mechanisms by which the resetting of the IHR occurred.

\section{Mechanism of Induction of Increased Intrinsic Heart Rate}

\section{a. Reserpine Inhibition}

The only well known mechanism by which HR is increased is via an increase in the activity of the sympathetic nervous system or a withdrawal of parasympathetic tone. Previous studies have indicated that, in response to endotoxin, there is a surge in catecholamine levels. It was hypothesized that inhibition of this increase in catecholamine levels would prevent the increase in IHR. Appropriate doses of reserpine, an inhibitor of adrenergic vesicular uptake, are known to deplete an animal of catecholamines over time. Reserpine treatment was employed as a means of depleting animals of catecholamines and thereby preventing the increase in HR induced by endotoxemia. Using a dosing regimen that has previously been demonstrated to deplete $95 \%$ of the myocardial catecholamines in rats, $1 \mathrm{mg} / \mathrm{kg} /$ day for 2 days (Rice et. al., 1987) we examined HR following LPS administration. Animals were dosed 32 and 8 hours prior to the administration of endotoxin with $1 \mathrm{mg} / \mathrm{kg}$ reserpine or sham vehicle. Equal numbers from each group then received either LPS $(5 \mathrm{mg} / \mathrm{kg})$ or saline sham. Sixteen hours later the animals were anesthetized and the in vivo HR recorded. The hearts were then extirpated and perfused as working left heart preparations and in vitro HR recorded. As demonstrated in figures 14 and 15, reserpine pretreatment abolished both the in vivo and in vitro differences in HR induced by LPS. 


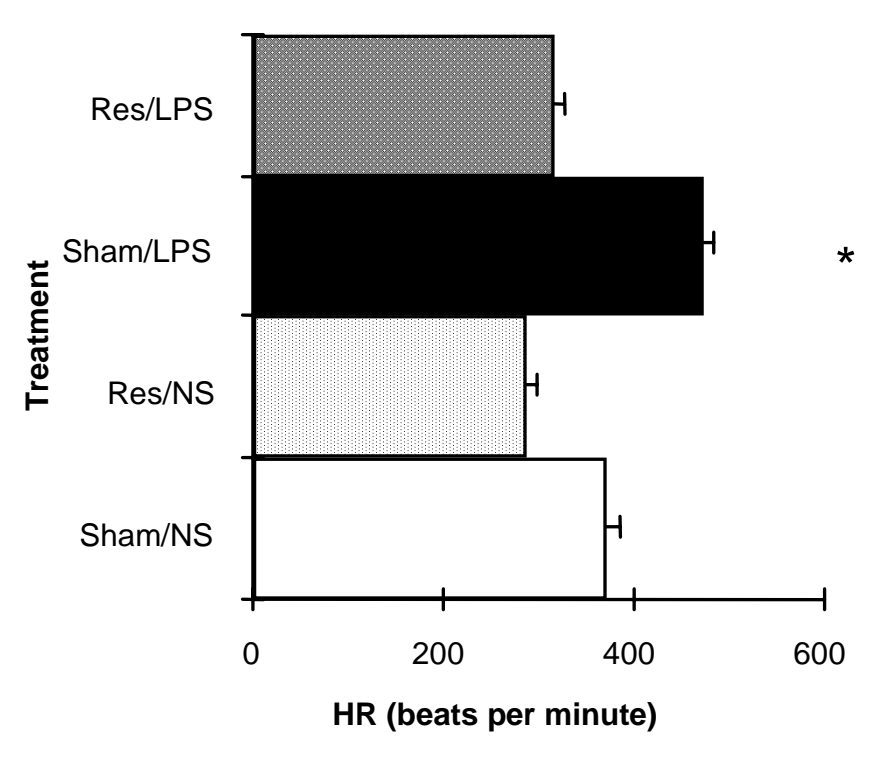

Figure 14. In vivo HR $16-20$ hours after LPS (5 mg/kg) or saline (NS) sham. Thirty-two and 8 hour prior to LPS animals received reserpine (Res, $1 \mathrm{mg} / \mathrm{kg}$ ) or an equal volume of sham vehicle (Sham). Error bars represent SEM. Sham/LPS is significantly greater than all other groups ( $p<0.05, n=6$ per group).

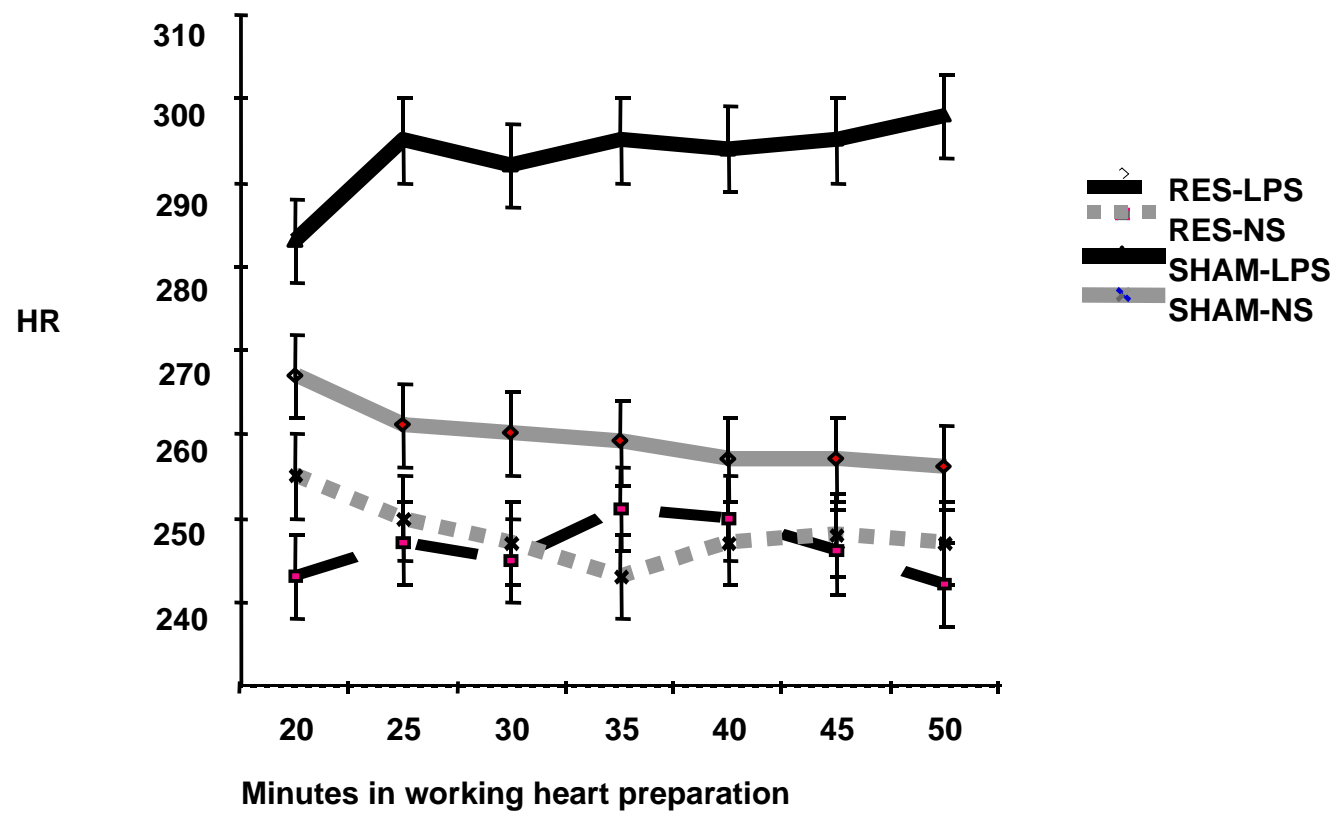

Figure 15. In vitro HR 16 - 20 hours after LPS (5 mg/kg) or saline (NS) sham. Thirtytwo and 8 hour prior to LPS animals received reserpine (Res, $1 \mathrm{mg} / \mathrm{kg}$ ) or an equal volume of sham vehicle (Sham). Error bars represent \pm SEM. Sham-LPS line is significantly greater than all other lines $(p=0.04, n=6$ per group). Other lines are not different from each other. 
In this experiment, using a statistical model to correct for differences in HR, no significant differences were observed among groups in the in vitro cardiac output (CO), coronary flow $(\mathrm{CF})$, velocity of aortic flow (A dP/dt) or cardiac output times peak systolic pressure $(\mathrm{CO} \mathrm{x}$ PSP), a measure of the ability of the heart to perform work (Table 5).

\begin{tabular}{|c|c|c|c|c|c|}
\hline & Sham-NS & Sham-LPS & Res-NS & Res-LPS & $\mathrm{p}$ \\
\hline $\mathrm{HR}(\mathrm{bpm})$ & $238 \pm 6$ & $282 \pm 6 *$ & $237 \pm 6$ & $246 \pm 7$ & 0.0482 \\
\hline $\mathrm{CO}(\mathrm{ml} / \mathrm{min})$ & $103 \pm 5$ & $89 \pm 6$ & $95 \pm 5$ & $97 \pm 4$ & 0.1559 \\
\hline $\mathrm{CF}(\mathrm{ml} / \mathrm{min})$ & $5.15 \pm .37$ & $6.07 \pm .50$ & $5.54 \pm .39$ & $5.98 \pm .33$ & 0.5360 \\
\hline $\mathrm{A} \mathrm{dP} / \mathrm{dt}$ & $1040 \pm 77$ & $1068 \pm 102$ & $1122 \pm 83$ & $1042 \pm 70$ & 0.3950 \\
\hline $\mathrm{CO}$ x PSP & $7567 \pm 387$ & $6351 \pm 400$ & $7438 \pm 362$ & $6966 \pm 362$ & 0.1384 \\
\hline
\end{tabular}

Table 5. In vitro cardiovascular parameters 16-20 after LPS (5 mg/kg, i.p.) or saline (NS). Equal numbers of animals were pretreated with reserpine (Res, $1 \mathrm{mg} / \mathrm{kg}$ ) or vehicle (Sham) 32 and 8 hours before LPS or NS. HR = heart rate, CO = cardiac output, $C F=$ coronary flow, $A d P / d t=$ aortic $d P / d t, C O \times P S P=$ cardiac output $x$ peak systolic pressure. Error is \pm SEM. The $p$ value represents the ANOVA for the combined treatment effect (Sham or Res combined with LPS or NS). * = significantly greater than all other treatments $p<0.0083$.

The administration of a single dose of reserpine 8 hours prior to endotoxin was not sufficient to abolish the endotoxin induced increase in in vitro HR observed 16 hours later (figure 16). This lack of effect was most likely due to insufficient depletion of catecholamines prior to the administration of endotoxin. 


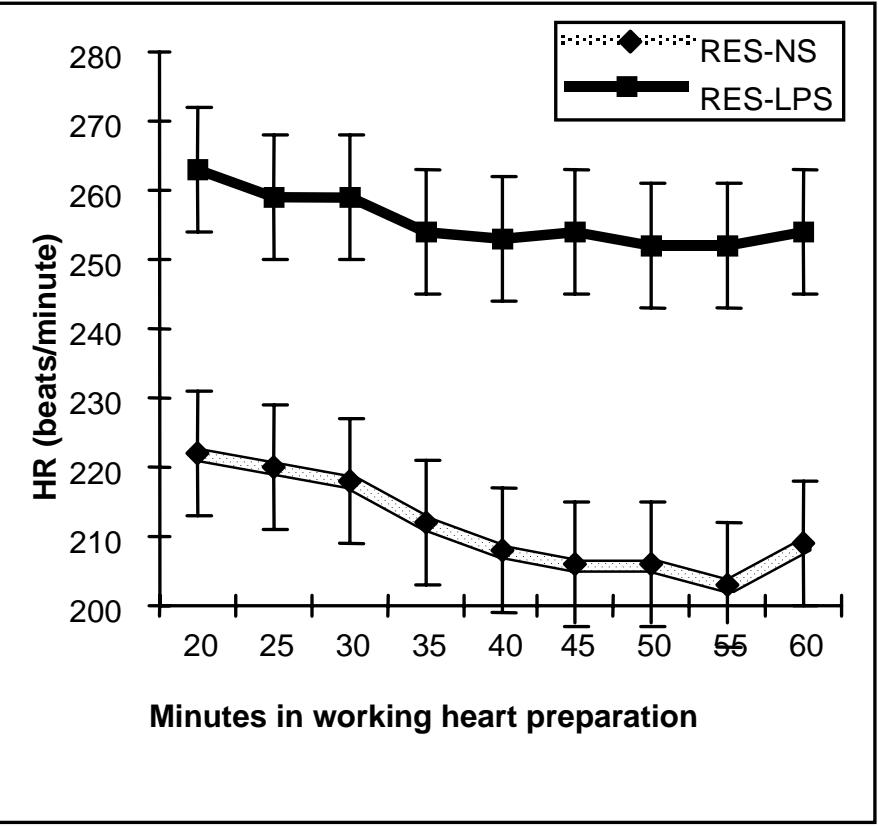

Figure 16. In vitro HR following a single dose of reserpine (RES, $2 \mathrm{mg} / \mathrm{kg}) 8$ hours prior to LPS or saline (NS) administration. Animals were sacrificed and hearts studied 16 hours after LPS or NS was administered in vivo. Error bars represent \pm SEM. The separation between the lines is approaching significance at $p=0.053(n=5 /$ group $)$.

The reserpine data demonstrated that substantive depletion of catecholamines prior to the administration of LPS could abolish the LPS induced increase in intrinsic heart rate. We hypothesized that the persistent elevation of the intrinsic heart rate must therefore be mediated by catecholamines. However, this increase outlasted the ongoing presence of catecholamines, as evidenced by the failure of propranolol to abolish either the in vivo or in vitro increase in HR once initiated. Reserpine can be directly toxic to the myocardium and it has been shown to cause depletion of other neurotransmitters, particularly serotonin or 5-HT (Heslop and Curzon, 1994; Iwayama et. al., 1973; Jandhyala et. al., 1971; Nagata et. al., 1987). To ascertain whether catecholamines caused the increase in intrinsic heart rate via the $\beta A R$, or whether reserpine abolished the difference in HR because of its noncatecholaminergic effects, animals were exposed to endotoxin during continual antagonism of the $\beta A R$. 


\section{b. Inhibition of increased intrinsic heart rate by $\beta$ adrenoceptor antagonism}

To determine if the effects of reserpine were due to depletion of other neurotransmitters or toxic effects of the agent, animals were pretreated with a $\beta \mathrm{AR}$ antagonist prior to exposure to endotoxin. If this experiment also abolished the increase in HR, the intrinsic tachycardia of sepsis was likely mediated by an effect of the sympathetic neurotransmitter via the $\beta$ AR. The proper dosing of reversible competitive antagonists in order to completely block the receptor for $16-20$ hours can be difficult to achieve. In this instance the problem is compounded by the markedly increased and varying levels of agonist, and the nonspecific effects of many $\beta A R$ antagonists at higher doses. An irreversible noncompetitive receptor antagonist is easier to employ because once adequate antagonism is achieved the duration of action depends only on the rate of receptor turnover. Bromoacetyl alprenolol menthane (BAAM) is an irreversible $\beta A R$ antagonist. BAAM has been reported to bind both $\beta 1$ and $\beta 2$ adrenoceptors with a duration of action of several days (Doggrell, 1990b). A dose of 35 $\mathrm{mg} / \mathrm{kg}$ has been demonstrated to block greater than $85 \%$ of the $\beta$ ARs in the rat heart, with receptor populations not returning to normal until 100 hours later (Baker and Pitha, 1982). Preliminary experiments were conducted in order to determine the appropriate dose for our model. We found that one hour after the administration of $40 \mathrm{mg} / \mathrm{kg}$ BAAM i.p. to naïve rats there was no response to $0.1 \mathrm{mg} / \mathrm{kg}$ of isoproterenol, a greater than maximal dose. Eight hours following a single dose of BAAM $(40 \mathrm{mg} / \mathrm{kg}$ ) decreased responsiveness to $\beta A R$ stimulation still remained (Figure 17). 


\section{Isoproterenol Dose Response Curve \\ Pre and 8 hours post BAAM}

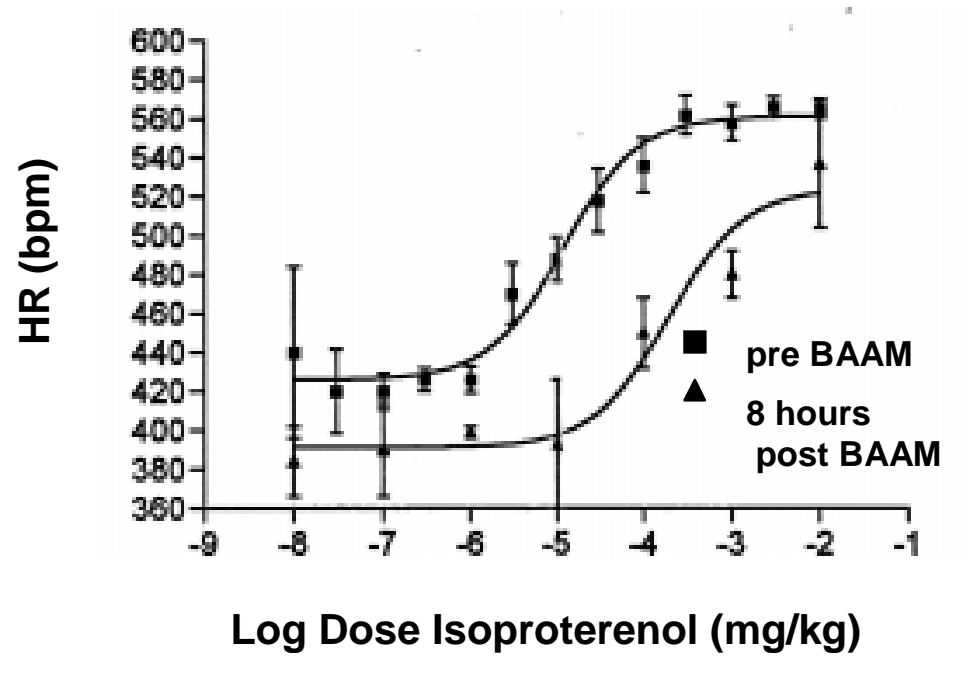

Figure 17. Heart rate (HR) response to i.v. isoproterenol in the conscious unrestrained rat 8 hours after a single dose BAAM (40 mg/kg i.p.) The isoproterenol ED50 pre-BAAM is $1.18 x$ $10^{-5} \mathrm{mg} / \mathrm{kg}$ and 8 hours post BAAM it is $1.92 \times 10^{-4} \mathrm{mg} / \mathrm{kg}(\mathrm{p}<0.05, n=3$ per group).

A separate group of animals was followed for 24 hours after a single i.p. dose of BAAM (40 $\mathrm{mg} / \mathrm{kg}$ ). Unlike the observations from the literature outlined in the introduction, we found that 24 hours after the administration of BAAM there was supersensitivity to isoproterenol (figure 18). These data should be interpreted with some caution, however, because the isoproterenol ED50 for BAAM administration in this group of animals was equal to the isoproterenol ED50 eight hours after BAAM in the previous series of experiments. This may reflect the small numbers of animals ( $n=3$ per group) used in these preliminary experiments. What is clear, is that 24 hours following a single dose of BAAM the HR response to isoproterenol was no longer inhibited. 


\section{Isoproterenol Dose Response Curve Pre and 24 hours Post BAAM}

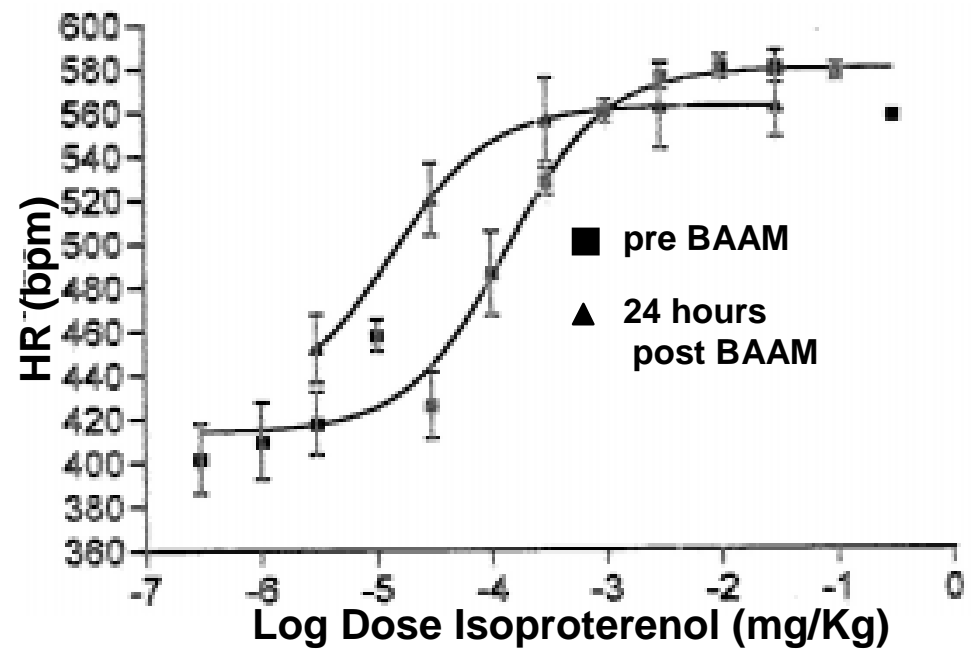

Figure 18. Isoproterenol dose response curve before and 24 hours after BAAM $(40 \mathrm{mg} / \mathrm{kg}$, i.p.) There is no change in maximum response. Isoproterenol ED50 pre-BAAM is $1.35 \times 10^{-}$ ${ }^{4} \mathrm{mg} / \mathrm{kg}$ and 24 hours post is $1.38 \times 10^{-5}$ ( $p<0.05, n=3$ per group $)$.

The goal of these experiments was to inhibit the $\beta A R$ until at least the point when the elevation in intrinsic heart rate was observed which was 16-20 hours after endotoxin. Knowing that the BAAM remained effective at 8 hours, a group of animals that had received BAAM was adminstered a second dose of BAAM $(40 \mathrm{mg} / \mathrm{kg})$ eight hours after the first dose. Twelve hours following this second dose, or twenty hours after the first dose, HR dose response curves to isoproterenol were repeated. Using this dosing regimen the HR response to isoproterenol was still decreased at 20 hours (Figure 19). 


\section{Isoproterenol Dose Response Curve \\ Pre and Post BAAM (2 doses)}

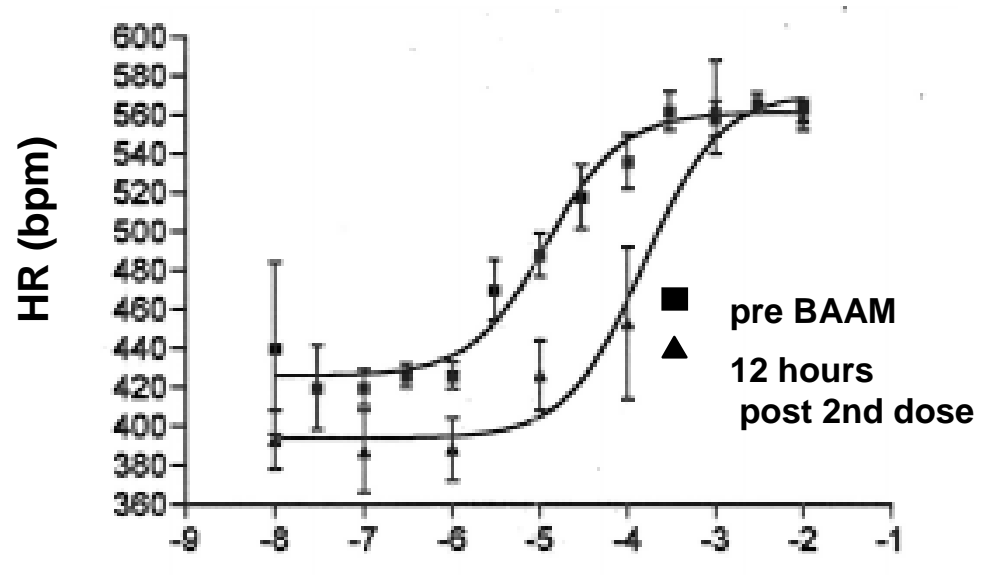

Log Dose Isoproterenol ( $\mathrm{mg} / \mathbf{k g})$

Figure 19. Heart rate response to i.v. isoproterenol in the conscious unrestrained rat 12 hours after a second dose of BAAM (40 mg/kg i.p.). The first dose had been given 20 hours earlier and was also $40 \mathrm{mg} / \mathrm{kg}$. There is no difference in the maximum response. The isoproterenol ED50 pre-BAAM was $1.185 \times 10^{-5} \mathrm{mg} / \mathrm{kg}$ and 8 hours post BAAM it is $1.5 \times 10^{-}$ ${ }^{4} \mathrm{mg} / \mathrm{kg}(\mathrm{p}<0.05, \mathrm{n}=3$ per group $)$.

The observation that the maximum response is not altered by BAAM suggests that either this agent is not irreversible or that there is a large $\beta \mathrm{AR}$ receptor reserve for the HR response. Because we had observed some lethality with the $40 \mathrm{mg} / \mathrm{kg}$ dose of BAAM increasing the dose was not a viable option. Instead we proceeded to see if this dosing regimen $(40 \mathrm{mg} / \mathrm{kg}$ every 8 hours $\mathrm{X}$ 2) would be adequate to prevent the increase in intrinsic heart rate in sepsis.

Animals were fitted with arterial catheters for conscious hemodynamic monitoring. Thirty minutes after the first dose of BAAM, endotoxin ( $5 \mathrm{mg} / \mathrm{kg}$, i.p.) or saline was administered. Blood pressure and HR were followed for 16-20 hours, the animals were then anesthetized, the hearts extirpated and in vitro cardiac function was examined. This dose of 
BAAM caused a significant amount of mortality outside of the combined effects with LPS (table 6).

\begin{tabular}{|c|c|c|c|c|c|}
\hline & BAAM-LPS & BAAM-NS & ET-LPS & ET-NS & TOTAL \\
\hline Mortality/Animals & $5 / 10$ & $2 / 8$ & $1 / 7$ & $0 / 5$ & $8 / 30$ \\
\hline$\%$ Mortality & $50 \%$ & $25 \%$ & $14 \%$ & $0 \%$ & $27 \%$ \\
\hline
\end{tabular}

Table 6. Mortality associated with BAAM or ethanol (ET) vehicle (40 mg/kg, i.p $\times 2$ doses) and LPS $(5 \mathrm{mg} / \mathrm{kg})$ or saline.

The HR response was examined for a three-hour baseline period prior to the administration of any drugs. At time zero BAAM or vehicle was first administered and then repeated at 8 hours. LPS was given 30 minutes following the first BAAM injection. Over all of the time periods there is a significant effect of treatment on HR $(p=0.0028)$ (figure 20).

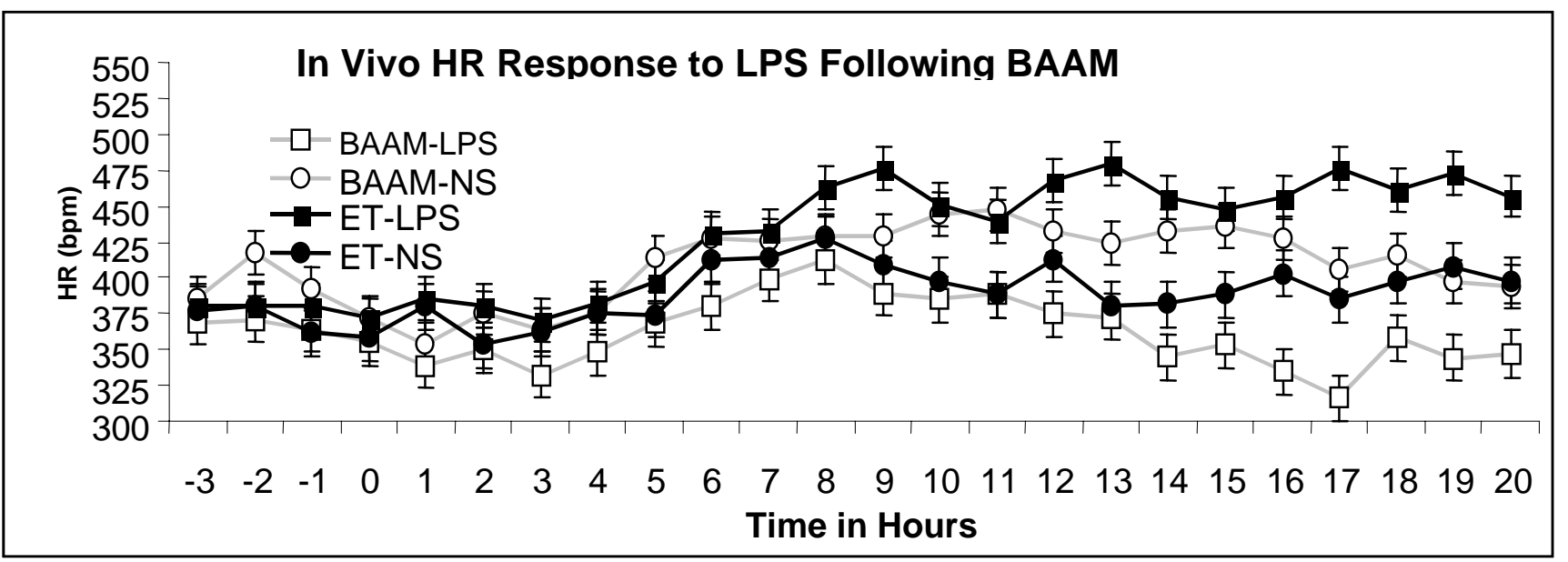

Figure 20. Heart rate response to LPS (5 $\mathrm{mg} / \mathrm{kg}$, i.p.) or saline (NS) following treatment with $B A A M(40 \mathrm{mg} / \mathrm{kg}$, i.p.) or ethanol vehicle (ET). A three hour baseline (time -3 to 0$)$ was observed prior to the administration of any drug. BAAM or ET was given at time 0 followed 30 minutes later by LPS or NS. BAAM or ET was repeated at time 8 hours. Error bars are \pm SEM.

There were no differences in mean arterial pressure (MAP) between treatment groups when examining all time periods (Figure 21). 


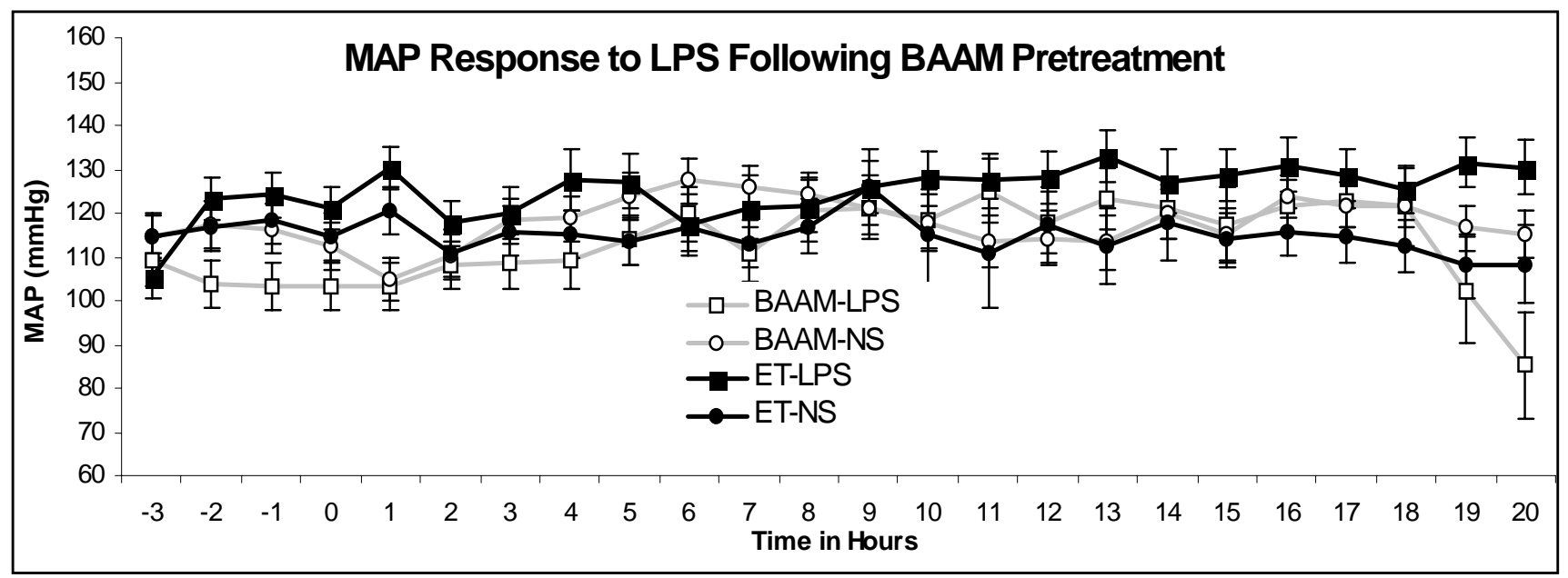

Figure 21. Mean arterial pressure (MAP) response to LPS (5 mg/kg, i.p.) or saline (NS) following treatment with BAAM (40 mg/kg, i.p.) or ethanol vehicle (ET). A three hour baseline (time -3 to 0 ) was observed prior to the administration of any drug. BAAM or ET was given at time 0 followed 30 minutes later by LPS or NS. BAAM or ET was repeated at time 8 hours. Error bars are \pm SEM.

A subset analysis was performed for the time baseline time period as well as time 16-20 hours. The analysis of variance showed that there were no baseline differences between groups with regard to heart rate $(\mathrm{p}=0.08)$ or blood pressure $(\mathrm{p}=0.15)$. At 16 to 20 hours, however, there was a significant HR $(\mathrm{p}=0.0039)$ differences between groups but no significant MAP differences $(\mathrm{p}=0.08)$ (Table 7).

\begin{tabular}{|l|l|l|l|l|}
\hline & \multicolumn{2}{|c|}{ HR (bpm) } & \multicolumn{2}{c|}{ MAP (mmHg) } \\
\hline & Baseline & $16-20$ hours & Baseline & $16-20$ hours \\
\hline BAAM-LPS & $368 \pm 9$ & $328 \pm 31^{*}$ & $105 \pm 4$ & $113 \pm 8$ \\
\hline BAAM-NS & $399 \pm 8$ & $408 \pm 13 \#$ & $115 \pm 4$ & $120 \pm 3$ \\
\hline ET-LPS & $380 \pm 8$ & $463 \pm 19$ & $118 \pm 4$ & $129 \pm 4$ \\
\hline ET-NS & $374 \pm 9$ & $392 \pm 23 \#$ & $117 \pm 4$ & $113 \pm 6$ \\
\hline
\end{tabular}

Table 7. Heart rate (HR) and mean arterial pressures (MAP) at baseline and 16-20 hours after endotoxin (LPS, $5 \mathrm{mg} / \mathrm{kg}$ ) or saline (NS). Animals received BAAM (40 $\mathrm{mg} / \mathrm{kg}$ ) or vehicle (ET) 30 minutes before and 8 hours after LPS or NS. Error is \pm SEM and $n=5$ to 6 per group. * = significantly less than ET-LPS $(p<0.002)$. \# = significantly less than ET$\operatorname{LPS}(p<0.03)$. 
At the completion of the in vivo portion of the experiment the animals were anesthetized and the hearts extirpated and perfused as working left heart preparations. After a 20 minute stabilization period the heart rate, cardiac output and coronary flow were recorded every 5 minutes for 20 minutes. The statistical model $\left(\mathrm{R}^{2}=0.95\right)$ demonstrated a significant difference between treatments $(\mathrm{p}=0.036)$. As demonstrated below, the ET-LPS heart rate was the greatest and BAAM-LPS the lowest, apparently confirming that BAAM would prevent an in vitro elevation of HR (figure 22). However, the conservative method of multiple comparison (Bonferroni) that was used requires a p value (Student's t-test) less than 0.0083 to demonstrate significant differences for an $\alpha=0.05$ if six comparisons are made $(0.05 / 6=0.083)$. By this conservative method no significant differences were detected by multiple comparison. This is most likely related to the small numbers of animals (5 to 6) per group. In general the statistical model we have utilized requires at least 6 animals per group for sufficient power. For the reader's benefit the results of the student's t-tests between groups are also shown (table 8).

\begin{tabular}{|c|c|c|c|c|}
\hline & BAAM-LPS & BAAM-NS & ET-LPS & ET-NS \\
\hline BAAM-LPS & $\mathbf{( 2 8 1} \pm \mathbf{2 3})$ & 0.4211 & $0.0198^{*}$ & 0.8071 \\
\hline BAAM-NS & 0.4211 & $\mathbf{( 3 0 6} \pm \mathbf{2 1})$ & 0.087 & 0.5538 \\
\hline ET-LPS & $0.0198^{*}$ & 0.087 & $\mathbf{( 3 5 9} \pm \mathbf{2 1})$ & 0.0264 \\
\hline ET-NS & 0.087 & 0.5538 & $0.0264^{*}$ & $\mathbf{( 2 8 9} \pm \mathbf{2 1})$ \\
\hline
\end{tabular}

Table 8. Numbers represent the p value from Student's t-tests comparing in vitro HR between treatment groups 16-20 hours after LPS or NS. The numbers in bold are the mean values \pm SEM for each treatment. These values apply to the lines in Figure 22. Animals received BAAM $(40 \mathrm{mg} / \mathrm{kg})$ or vehicle (ET) 30 minutes before and 8 hours after LPS or NS and $n=5$ to 6 per group. 


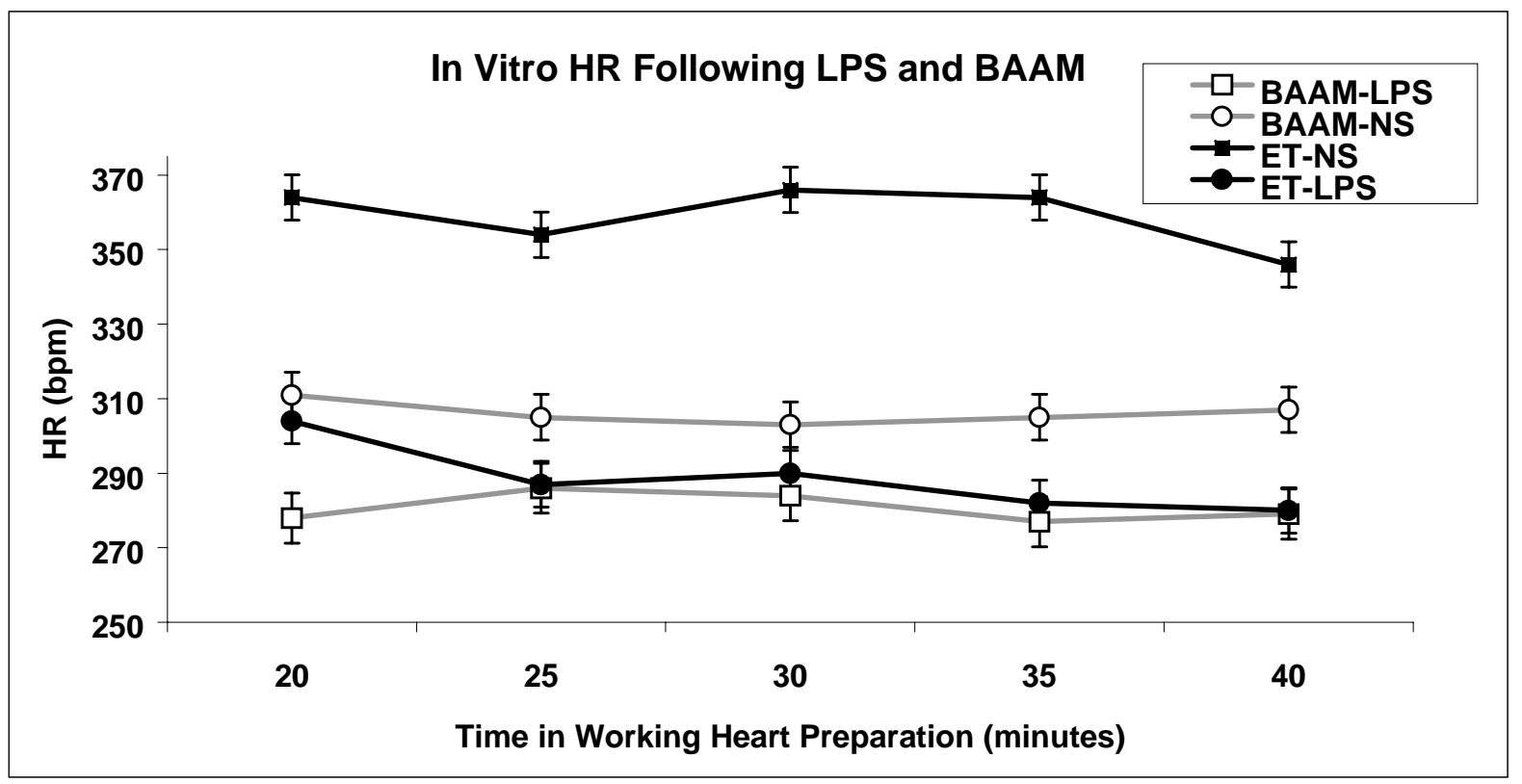

Figure 22. In vitro heart rate (HR) following in vivo LPS and BAAM. Animals received BAAM (40 mg/kg) or vehicle (ET) 30 minutes before and 8 hours after LPS or NS in vivo. A 20-minute stabilization period was observed. Error bars are \pm SEM. The effect of treatment was significant ( $p=0.036, n=5$ to 6 per group).

At this time point (16-20 hours after LPS) the ET-LPS in vitro HR is $17 \%$ greater than the control animals (ET-NS). This is consistent with the previous observations. Furthermore, the ET-LPS in vitro HR is $24 \%$ greater than the BAAM-LPS. The experimental evidence strongly suggests that pretreatment with BAAM inhibited the LPS induced increase in intrinsic heart rate. This observation supports the conclusion that the action of reserpine in preventing the same elevation of HR was due to the depletion of catecholamines and the subsequent inability of neurotransmitter to stimulate the $\beta \mathrm{AR}$.

Coronary flow $(\mathrm{CF})$ and cardiac output $(\mathrm{CO})$ were also examined in the in vitro preparation. The statistical model, which controlled for the variation in HR, detected significant differences with respect to treatment for CF $(p=0.0378)$. There were no treatment differences with regard to $\mathrm{CO}(\mathrm{p}=0.0604)$. The BAAM-LPS group had the lowest value for both variables (table 9). 


\begin{tabular}{|l|l|l|l|l|}
\hline & BAAM-LPS & BAAM-NS & ET-LPS & ET-NS \\
\hline Coronary Flow (ml/min) & $12.4 \pm 1.4$ & $20.2 \pm 0.8^{*}$ & $20.6 \pm 2.2$ & $19.1 \pm 1.3^{*}$ \\
\hline Cardiac Output (ml/min) & $72.3 \pm 5.6$ & $99.8 \pm 3.3$ & $92.56 \pm 8.7$ & $86.4 \pm 5.0$ \\
\hline
\end{tabular}

Table 9. Coronary flow and cardiac output in vitro following LPS and BAAM. Animals received BAAM (40 mg/kg) or vehicle (ET) 30 minutes before and 8 hours after LPS or NS in vivo. Error is \pm SEM. ${ }^{*}=$ significantly greater than BAAM-LPS $p<0.0083, n=5$ to 6 per group.

BAAM antagonizes the $\beta 2$ receptors, which mediate vasodilitation, as well as the $\beta 1$ receptors which mediate the majority of myocardial effects. The finding of decreased coronary flow in the BAAM-LPS group suggests that released catecholamines may also play a role in preserving $\mathrm{CF}$ in sepsis. This finding was not observed in the reserpine - LPS group. The reserpine, however would also have inhibited $\alpha$ - adrenoceptor mediated vasoconstriction of arterioles. Thus, BAAM may influence $\mathrm{CF}$ through an alteration of the balance between these two processes. The decreased CF could account for the somewhat lower CO observed in the BAAM-LPS group.

To examine whether BAAM was still effective, in terms of $\beta$ AR antagonism, at the time of these observations an isoproterenol concentration response curve was constructed for the in vitro hearts. The HR concentration response curve is shown below (figure 23). There was no difference in the $\mathrm{HR}$ response to $\beta \mathrm{AR}$ stimulation based upon the calculation of the geometric mean EC50 and 95\% confidence intervals (table 10). 


\section{Isoproterenol Concentration Response Following BAAM}

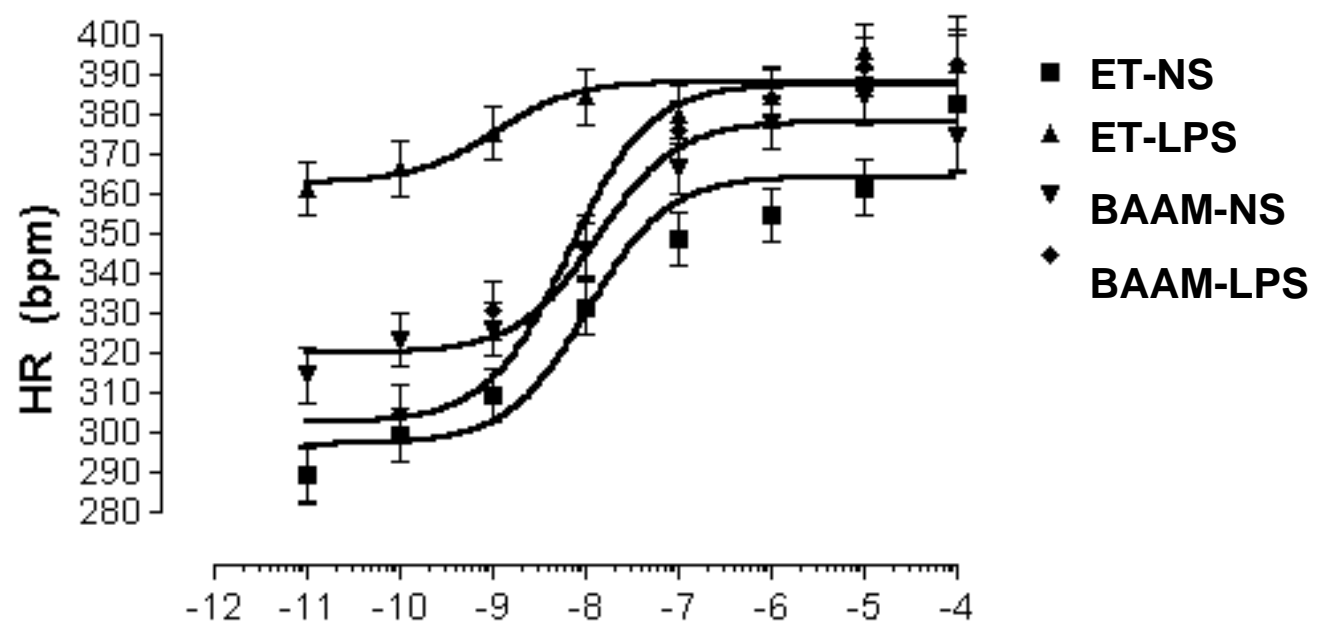

Log Concentration Isoproterenol (M)

Figure 23. Heart rate (HR) concentration response curve to isoproterenol in vitro. Animals received BAAM (40 $\mathrm{mg} / \mathrm{kg})$ or vehicle (ET) 30 minutes before and 8 hours after LPS or NS in vivo. Error is \pm SEM. There is no significant difference in maximum response.

\begin{tabular}{|c|c|c|}
\hline & Mean EC50 & $95 \%$ C.I. \\
\hline BAAM-LPS & $5.7 \times 10^{-9}$ & $3.7 \times 10^{-10}$ to $8.9 \times 10^{-8}$ \\
\hline BAAM-NS & $3.2 \times 10^{-8}$ & $6.0 \times 10^{-9}$ to $1.7 \times 10^{-7}$ \\
\hline ET-LPS & $2.5 \times 10^{-9}$ & $1.1 \times 10^{-10}$ to $5.6 \times 10^{-8}$ \\
\hline ET-NS & $4.6 \times 10^{-8}$ & $1.4 \times 10^{-9}$ to $1.6 \times 10^{-6}$ \\
\hline
\end{tabular}

Table 10. Geometric mean EC50 and $95 \%$ confidence interval for HR response to isoproterenol. Animals received BAAM (40 mg/kg) or vehicle (ET) 30 minutes before and 8 hours after LPS or NS in vivo. The confidence intervals all overlap.

Based upon these data, the $\beta$ AR was no longer significantly antagonized by BAAM at a time at which the in vitro HR was elevated. This implies that the $\beta \mathrm{AR}$ was fully functional in the BAAM-LPS group and yet neither the in vitro nor the in vivo HR was elevated in these animals. This provides further evidence that functional $\beta$ ARs are required at an early stage in the septic process to increase HR but that the later persistent elevation in HR is not dependent upon continued $\beta A R$ activity. 


\section{c. Response of reserpinized animals exposed to maximal doses of $\beta A R$ agonist}

The question arises as to whether stimulation of the $\beta \mathrm{AR}$ is required in tandem with LPS itself, or the accompanying cytokine elevations, early in the septic process to induce the elevation of IHR. An alternative hypothesis would be that LPS induces a change in the cellular processes controlling IHR that only becomes apparent after stimulation of the $\beta$ AR. If the latter hypothesis were true animals exposed to reserpine and then LPS should develop an elevation of the IHR if they were subsequently exposed to a $\beta$ AR agonist. In order to test this hypothesis animals were pretreated with reserpine as above $(1 \mathrm{mg} / \mathrm{kg} 32$ and 8 hours prior to LPS or NS) and then treated with LPS. Sixteen hours after LPS the right atria were excised and placed into organ baths. After a 40-minute stabilization period an isoproterenol concentration response curve was constructed. The isoproterenol was then rinsed from the bath and the heart rate decay over the following one-hour was examined and compared to control animals. The treatment groups consisted of Sham-NS, Sham-LPS, Reserpine-NS and Reserpine-LPS.

The in vivo HR was significantly higher in the Sham-LPS group than the reserpineNS or the reserpine-LPS groups. There were no statistically significant differences in the basal beating rates of the isolated right atria. The Sham-LPS rate was, however, $17 \%$ greater than the Sham-NS which is in agreement with the previous observations. There were also no statistical differences in the maximum rate response to isoproterenol or the beating rate onehour after the removal of isoproterenol from the bath (Table 11). 


\begin{tabular}{|c|c|c|c|c|}
\hline Treatment & In Vivo HR & $\begin{array}{c}\text { Isolated Right } \\
\text { Atria Basal HR }\end{array}$ & $\begin{array}{c}\text { Isolated Right } \\
\text { Atria Max. HR }\end{array}$ & $\begin{array}{c}\text { Isolated Right } \\
\text { Atria Final HR }\end{array}$ \\
\hline Sham-NS & $399 \pm 21$ & $253 \pm 18$ & $455 \pm 18$ & $285 \pm 16$ \\
\hline Sham-LPS & $453 \pm 21$ & $295 \pm 18$ & $418 \pm 18$ & $331 \pm 16$ \\
\hline Reserpine-NS & $318 \pm 21^{*}$ & $238 \pm 18$ & $414 \pm 18$ & $300 \pm 16$ \\
\hline Reserpine-LPS & $320 \pm 21^{*}$ & $274 \pm 18$ & $428 \pm 18$ & $325 \pm 16$ \\
\hline
\end{tabular}

Table 11. In vivo HR and HRs of isolated right atria 16-20 hours after LPS (5mg/kg) or saline (NS) sham. Animals were pretreated with reserpine or vehicle (sham) 32 and 8 hours prior to excision of the right atria. Isolated right atria basal HR represents the basal beating rate 40 minutes after excision. The isolated right atria max. HR is the maximum $H R$ following maximal concentrations of isoproterenol. The isolated right atria final HR represents the HR one hour after isoproterenol washout. * = significantly different from Sham-LPS $p<0.05$.

The isoproterenol concentration response curves as absolute values of beating rate are demonstrated in Figure 24. The curves were also normalized as a percentage of the maximal increase in beating rate to adjust for the differences in basal rate (Figure 25). As can be seen from the curves there is no difference in the responsiveness of the isolated atria to isoproterenol regardless of treatment. The $\mathrm{EC}_{50}$ for the individual treatment groups are shown in table 12. 


\section{Isoproterenol Response Isolated Right Atria}

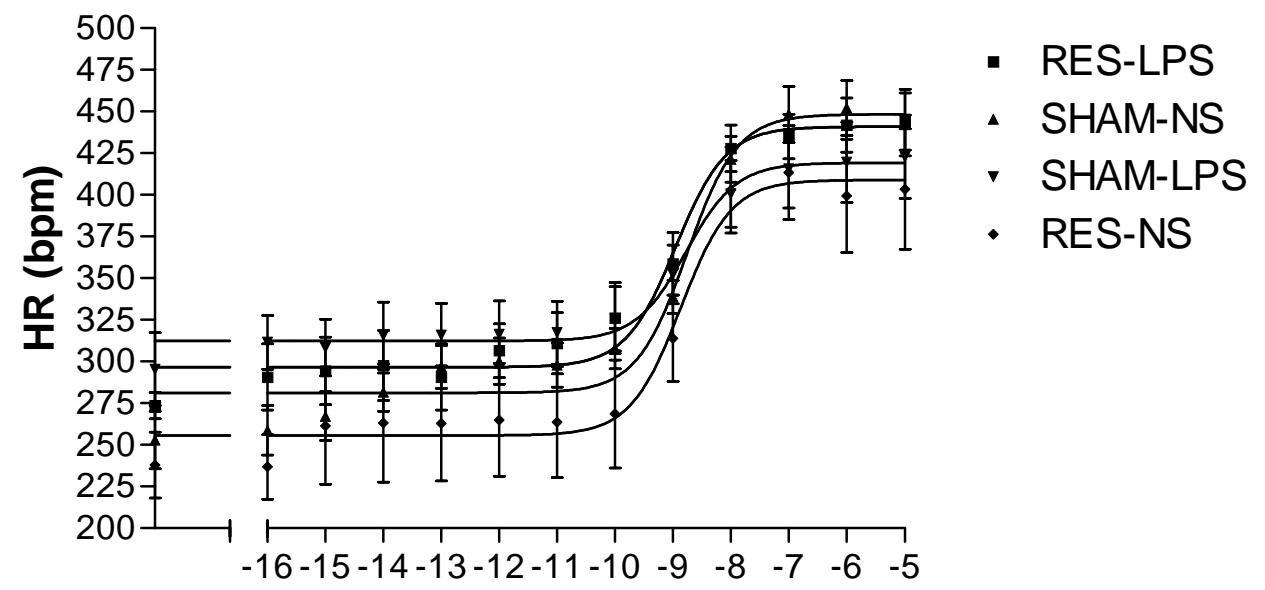

Log Concentration Isoproterenol

Figure 24. Heart rate (HR) concentration response curve to isoproterenol in isolated right atria. Animals received LPS or NS 16-20 hours prior. Animals were also pretreated with reserpine (RES) or vehicle (SHAM) 32 and 8 hours prior to LPS or NS. Error is \pm SEM .

\section{Isoproterenol Response Isolated Right Atria}

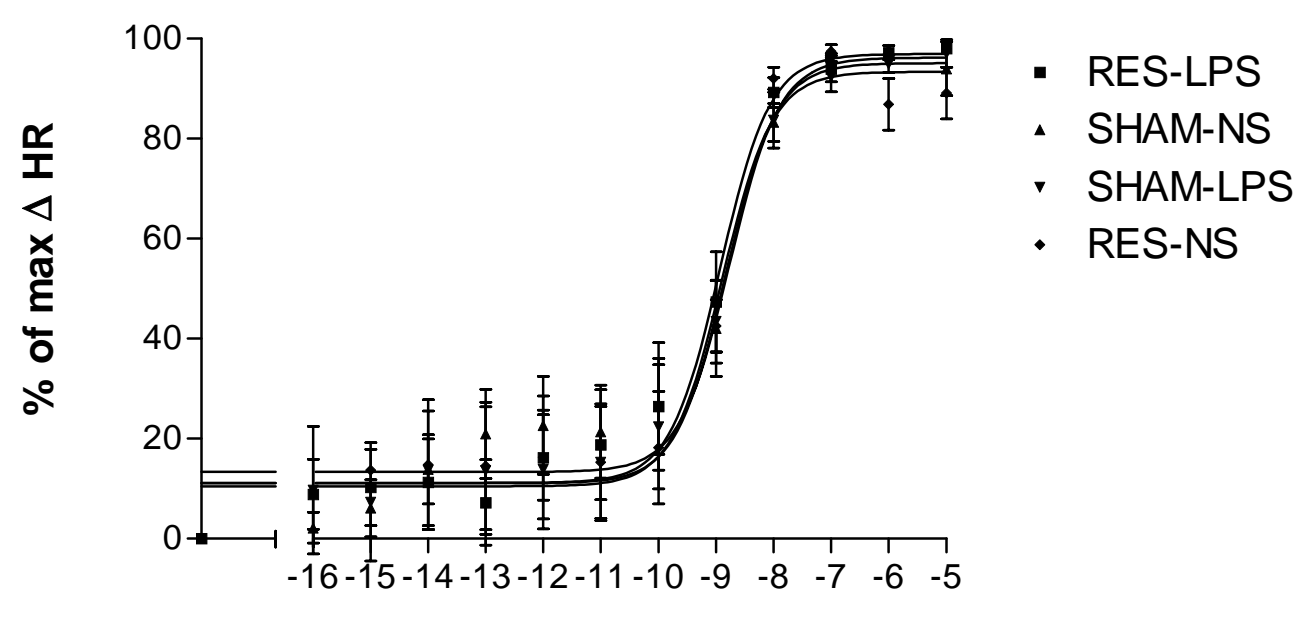

Log Concentration Isoproterenol

Figure 25. Percentage of maximal change in beating rate concentration response curve to isoproterenol in isolated right atria. Animals received LPS or NS 16-20 hours prior. Animals were also pretreated with reserpine (RES) or vehicle (SHAM) 32 and 8 hours prior to LPS or NS. Error is \pm SEM. 


\begin{tabular}{|c|c|c|c|c|}
\hline & \multicolumn{2}{|c|}{ Absolute Beating Rate } & \multicolumn{2}{c|}{ \% of Maximum Increase in Rate } \\
\hline & EC $_{50}$ & $95 \%$ CI & EC $_{50}$ & $95 \%$ CI \\
\hline Sham-NS & $1.743 \times 10^{-9}$ & $8.22 \times 10^{-10}$ to $3.40 \times 10^{-9}$ & $1.716 \times 10^{-9}$ & $8.53 \times 10^{-10}$ to $3.45 \times 10^{-9}$ \\
\hline Sham-LPS & $1.675 \times 10^{-9}$ & $3.74 \times 10^{-10}$ to $7.51 \times 10^{-9}$ & $1.499 \times 10^{-9}$ & $6.20 \times 10^{-10}$ to $3.62 \times 10^{-9}$ \\
\hline RES-NS & $1.362 \times 10^{-9}$ & $2.86 \times 10^{-10}$ to $6.48 \times 10^{-9}$ & $1.288 \times 10^{-9}$ & $5.89 \times 10^{-10}$ to $2.36 \times 10^{-9}$ \\
\hline RES-LPS & $8.854 \times 10^{-10}$ & $2.98 \times 10^{-10}$ to $2.63 \times 10^{-9}$ & $1.179 \times 10^{-9}$ & $8.22 \times 10^{-10}$ to $3.40 \times 10^{-9}$ \\
\hline
\end{tabular}

Table 12. Geometric mean $E C_{50}$ and $95 \%$ confidence intervals for the HR response of isolated right atria to isoproterenol. Animals were pretreated with reserpine (RES) or vehicle (Sham) and then received LPS or NS. The absolute beating rate is taken from figure 24. The \% of maximum increase in rate is taken from figure 25. All confidence intervals overlap.

The Sham-NS group did demonstrate a significantly steeper rate of decline from maximum beating rate than the other three groups (Figures 26 and 27). This finding relates mostly to the slightly higher maximum beating rate and the slightly lower finishing rate.

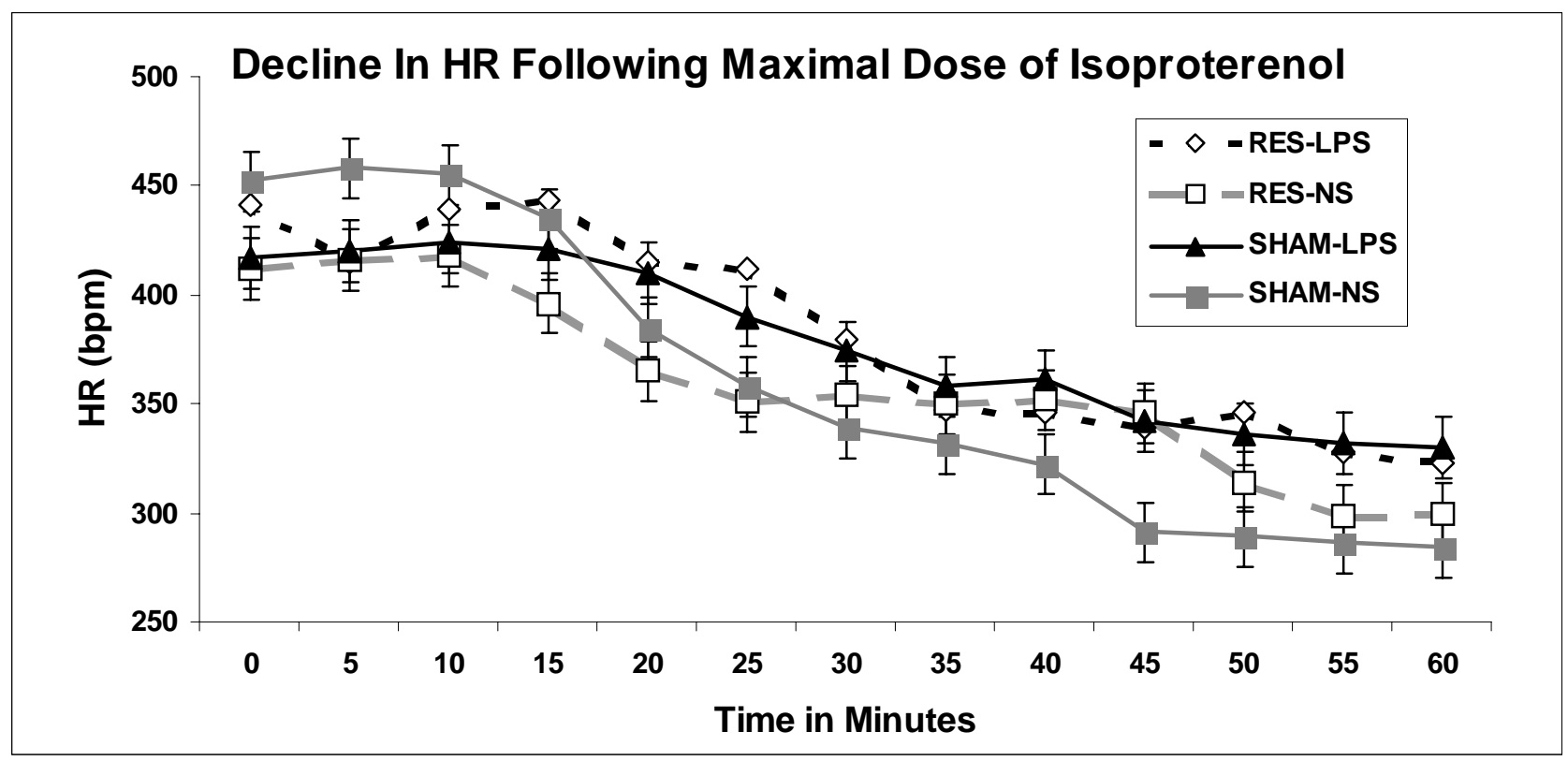

Figure 26. Decline in HR of isolated right atria from a maximal concentration of isoproterenol. Animals were pretreated with reserpine (RES) or vehicle (SHAM) 32 and 8 hours prior to the administration of LPS or NS. The right atria were excised 16-20 hours later and exposed to maximal concentrations of isoproterenol. Following removal of isoproterenol from the bath the decrease in HR was observed over the next one hour. The rate of decline of SHAM-NS is significantly steeper than the other three groups $(p<0.05)$. 


\section{Decline of HR Following Exposure to a Maximum Concentration of Isoproterenol - Isolated Right Atria}

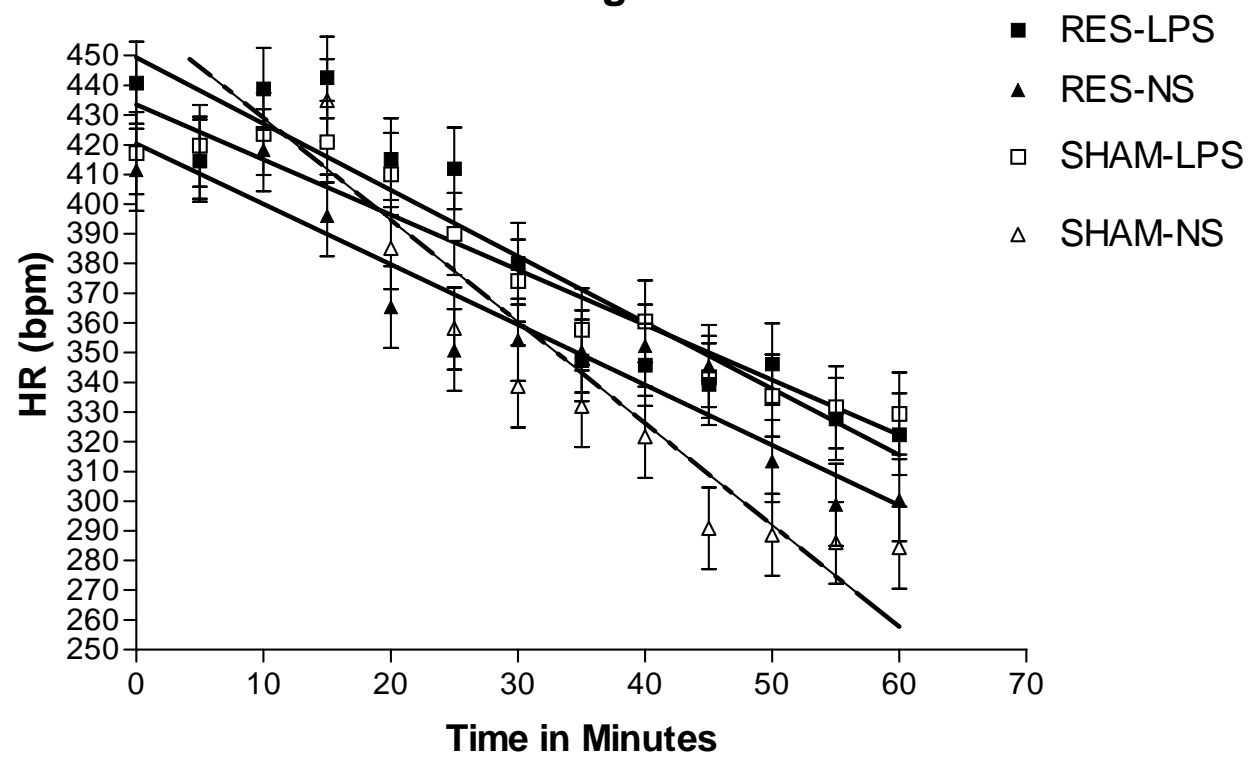

Figure 27. Decline in HR following exposure of isolated atria to a maximum concentration of isoproterenol. The Sham-NS (broken) line is significantly different from the others $p<0.05$. The $R^{2}$ for each of the lines is Sham-NS $=.93$, Sham-LPS $=.94$, Res-NS $=.92$ and Res-LPS $=.89$

Because none of the finishing rates have returned to baseline it is difficult to draw conclusions as to whether exposure to isoproterenol would elevate the IHR (in this case the final beating rate) of the reserpine-LPS group. The similarity of the rate of decrease of the four groups suggests that there is likely no difference in the decay of the response in the treatment groups with the exception of Sham-NS. While it is possible that both reserpine and LPS had an effect to slow the decline in HR following exposure of isolated right atria to a maximum concentration of isoproterenol, the fact that none of the groups returned to the baseline beating rate suggests that observation for a longer period of time is warranted prior to drawing this conclusion. The experiment does conclusively demonstrate that there is no difference in responsiveness with regard to the reserpine pretreatment or the LPS exposure. 


\section{d. Time course of changes in the catalytic subunit of protein kinase A and phospho-CREB}

Protein kinase A (PKA) exists in the inactivated state as a heterodimer composed of two regulatory subunits (PKA-R) and two catalytic subunits (PKA-C). Following the activation of adenylyl cyclase cAMP is produced. Each regulatory subunit of PKA can bind two molecules of cAMP (Cooper, 1997). Once four molecules have been bound to the heterodimer the catalytic subunits are released from the regulatory subunits and PKA-C becomes active. It is known the PKA-C can act in two ways. It can remain in the cytoplasm where it phosphorylates a variety of proteins or it can enter the nucleus where it phosphorylates and activates transcription factors in the cyclic AMP response element binding protein (CREB) (Tasken et. al., 1997). PKA-C remains active until it is rebound by PKA-R or until it is degraded by proteases (Hemmings, 1986). Alterations in the levels of PKA-C have been reported to correlate with the activation of the catalytic subunit by cAMP (Boundy et. al., 1998; Garrel et. al., 1997). In order to determine the time course of these changes and whether levels of PKA-C are likely to play a role in the observed elevation in IHR quantitative western blots were performed. Animals were sacrificed at 2, 4, 8 and 16 hours after LPS or NS pretreatment. Their right atria were processed for quantitative western blots and density was determined via densitometry. All observations were normalized as a percentage of the controls ran on the same blot. At two hours after LPS, PKA-C was $113 \% \pm$ $14 \%$ of control, at four hours it was $128 \% \pm 13 \%$ of control and at eight hours PKA-C was $112 \% \pm 14 \%$ of control. None of these values are significantly different from control levels. Sixteen hours after LPS, PKA-C levels in the right atrium had decreased significantly to $36 \%$ $\pm 14 \%$ of control values. Figure 28 demonstrates a representative western blot and figure 29 provides a graphic depiction of the above values. 


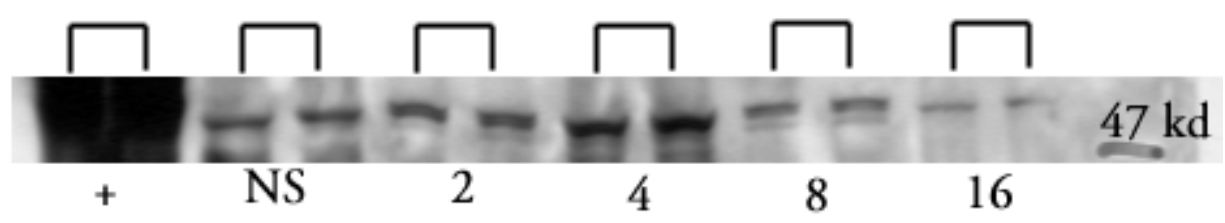

Figure 28. Representative western blot of PKA-C in the right atrium following the administration of endotoxin. $+=$ positive control, $N S=$ normal saline and 2, 4, 8, and 16 represent hours after endotoxin administration in vivo. Each well represents a separate animal.

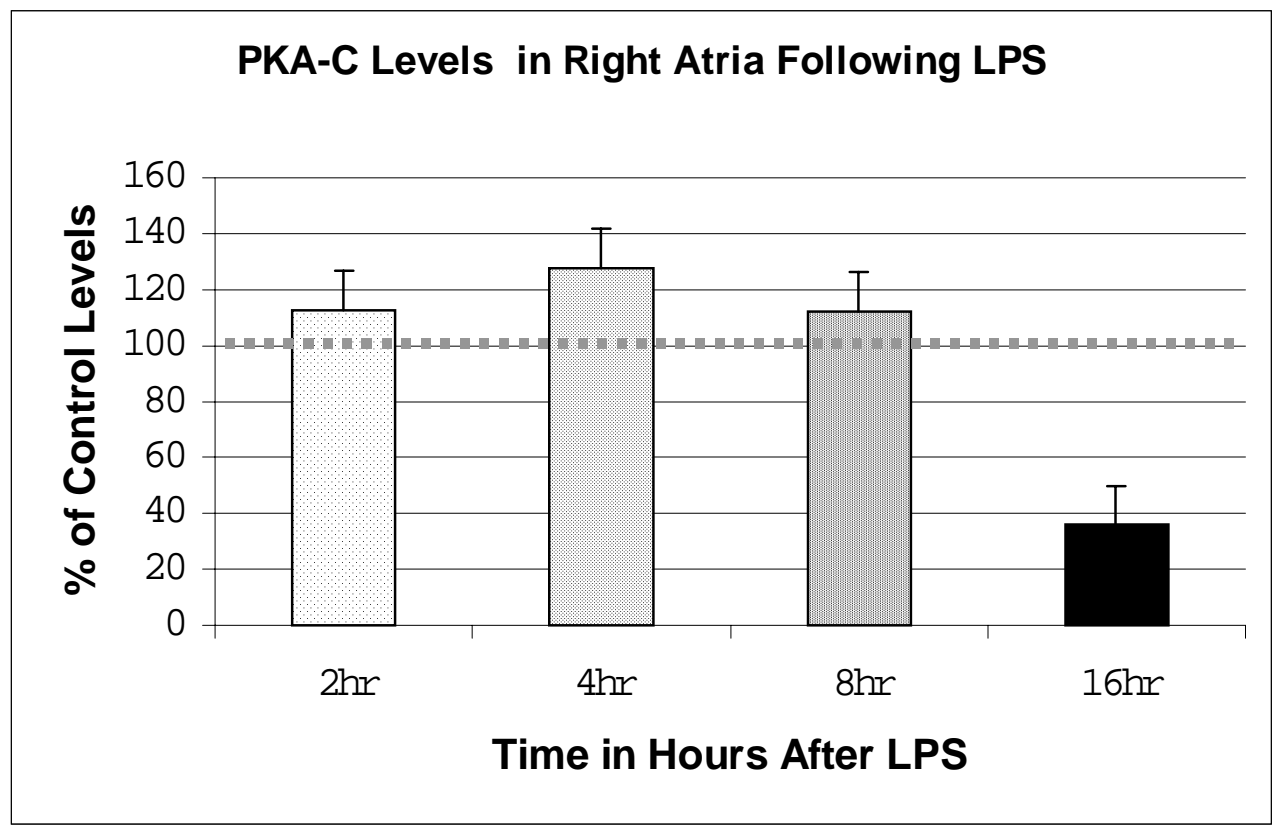

Figure 29. PKA-C levels in right atria following LPS administration in vivo. Only 16 hours is significantly different from control represented by the broken line ( $p<0.0001)$.

PKA-C levels are not necessarily reflective of PKA catalytic activity nor do they offer any information as to whether the message has been transduced to the nucleus or remained in the cytoplasm. The measurement of phosphorylated CREB does, however, offer information with regard to the degree to which, and time course over which, message has been transduced to the nucleus. For this reason an antibody specific for phosphorylated CREB was utilized in the same fashion on different aliquots from the same atria. 
Phosphorylated CREB levels had risen to $300 \%$ of control by two hours after LPS, reaching a peak of greater than $500 \%$ of control values by four hours and returning to baseline 16 hours after LPS administration. Figure 30 demonstrates a representative western blot and figure 31 provides a graphic depiction of the above values.

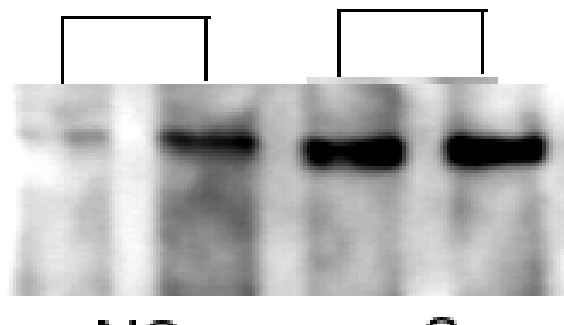

NS
2

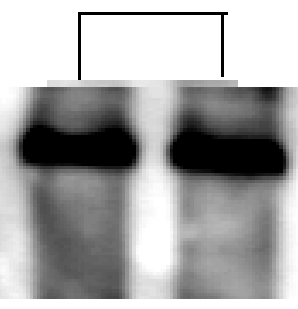

4

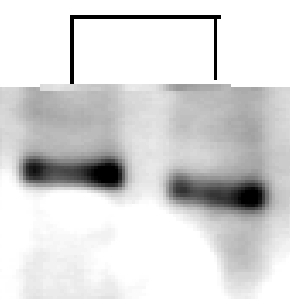

8

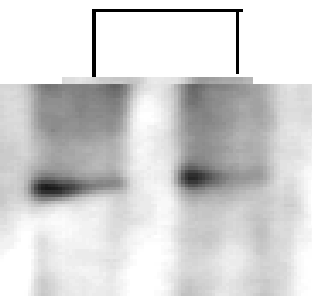

16

Figure30. Representative western blot of Phospho-CREB in the right atrium following the administration of endotoxin. NS = normal saline and 2, 4, 8, and 16 represent hours after endotoxin administration in vivo. Each well represents a separate animal.

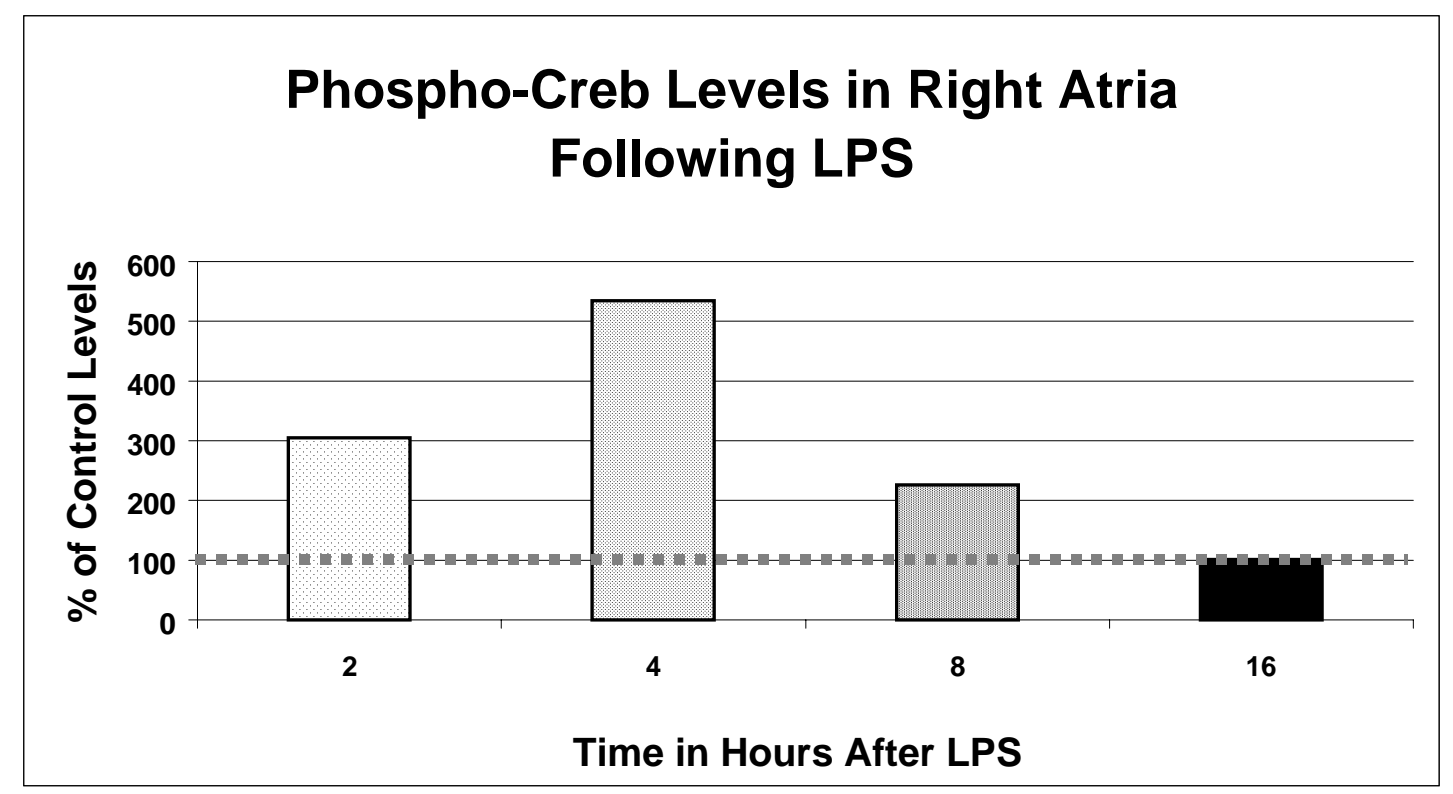

Figure 31. Phospho-CREB levels in right atria following LPS administration in vivo. The dotted line represents control. 


\section{e. Time Course of Changes in Heart Rate Variability}

Heart rate variability (HRV) has been reported to decrease in septic humans and rabbits. In order to determine if these changes also occurr in a rat model of endotoxemia and correlate with observed changes in IHR rats were fitted with subcutaneous ECG electrodes. Both time and frequency domain measurements of HRV were made prior to and following endotoxin administration in conscious animals. In agreement with the previous in vivo experiments the HR did not become elevated until four hours after endotoxin administration and it remained elevated through the observation period (figure 32).

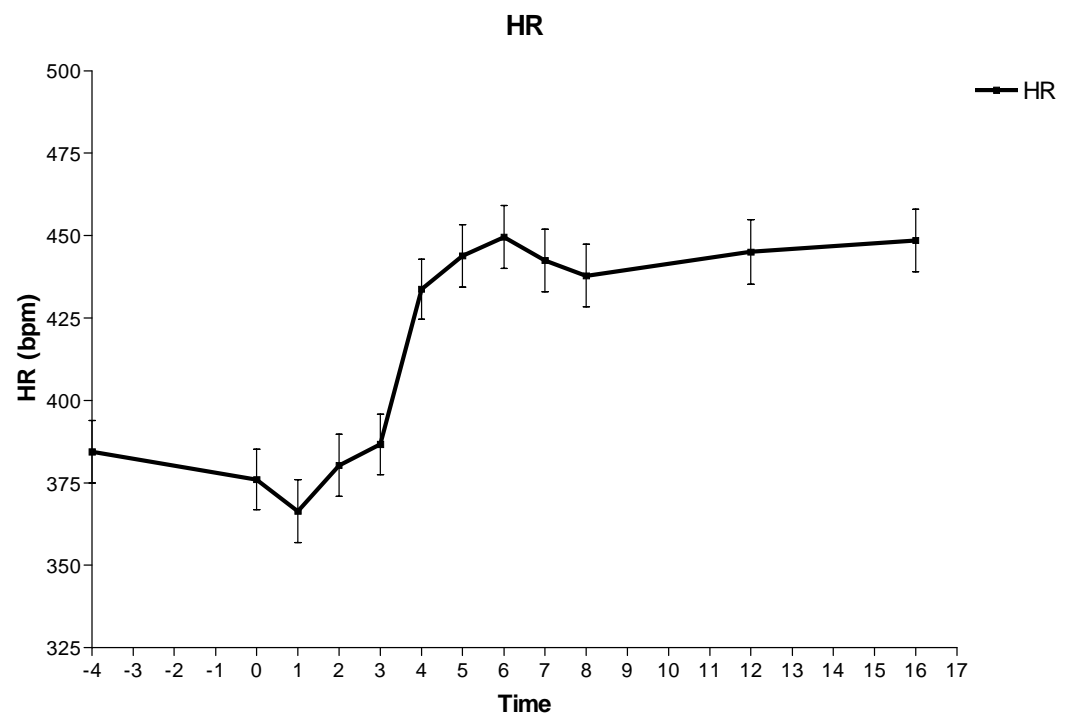

Figure 32. Changes in HR following the administration of LPS at time 0. Time is hours before or after LPS. HR becomes significantly elevated at 4 hours $(p<0.05)$ and remains elevated at 16 hours after LPS.

Measurements of total HRV included both total power and the standard deviation of all normal R-R intervals (SDNN) over five minute periods. Both of these measurements show a drop in HRV beginning two hours after LPS administration and reaching a maximum at 7 hours after LPS. Normal HRV had not returned at 16 hours after LPS (figures 33 and 34). 

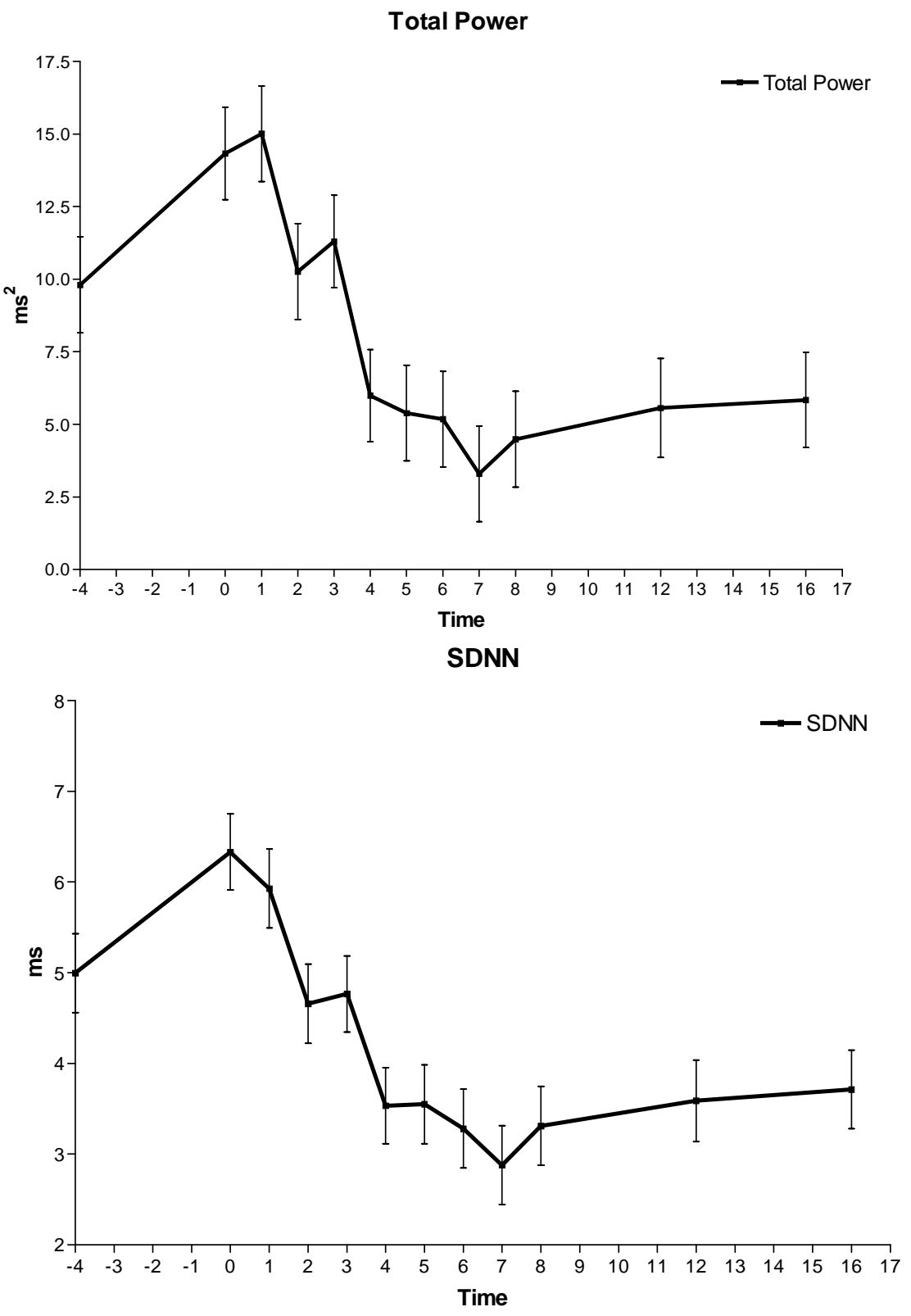

Figures 33 and 34. Total HRV as measured by Total power and SDNN measured for 5 minute intervals. LPS administration was at time 0. Time is equal to hours before and after LPS administration. In both measurements $H R V$ is significantly less than control at 2 hours after LPS $(p<0.05)$. HRV decreases further at 4 hours ( $p<0.05$ when compared to 2 hours) and then does not change through 16 hours after LPS. 
Parasympathetic tone was measured in both the time domain (RMSDD) and frequency domain (HF and HF nu) (Figures 35-37). An examination of RMSDD and HF suggests that there is an initial spike in parasympathetic activity one hour after the administration of LPS which then declines to below control levels by 4 hours after LPS and remains there throughout the experimental period. HF nu, which normalizes for the decrease in total HRV, suggests that parasympathetic tone increases at one hour and remains moderately elevated. Sympathetic tone, which can only be measured in the frequency domain (LF, LF nu and LF/HF) (figures 38-40), decreases significantly after LPS as measured by LF. LF nu and the $\mathrm{LF} / \mathrm{HF}$ ratio suggest that there is no change in sympathetic activity throughout the experimental period. These findings are consistent with those authors who found a decrease in HRV in sepsis (Garrard et. al., 1993; Godin et. al., 1996; Goldstein 1995). Furthermore the time course of changes agrees closely with our observations regarding intrinsic heart rate. With the exception of an early increase parasympathetic activity there appears to be minimal changes in autonomic tone throughout the experimental period. This may relate to the difficulty in measuring low frequency variability secondary to the large amount of artifact in the recordings. Our earlier findings with chemical antagonism of the $\beta A R$, however, also suggested minimal difference in the relative sympathetic tone in the control and endotoxin exposed state. Concern arises as to whether HRV as measured by SDNN and total power is directly related to HR. If this were the case an increase in HR may simply lower HRV due to their direct relationship. In order to answer this question linear regression was performed on HR against these measurements in the control and endotoxin treated state. Interestingly in the control state there is no relationship between the two but for the time periods after LPS there is a significant negative correlation $\left(\mathrm{R}^{2}=0.355, \mathrm{p}<0.0001\right)$. This further suggests that LPS increases the HR outside of the autonomic nervous system and consequently decreases the influence of this system on HRV. 


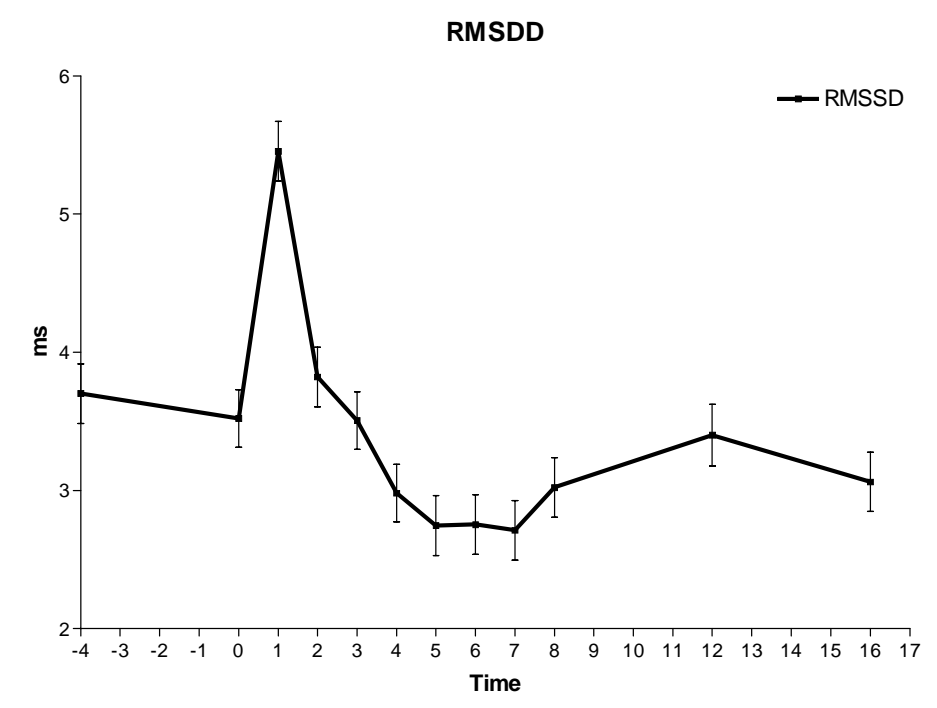

HF

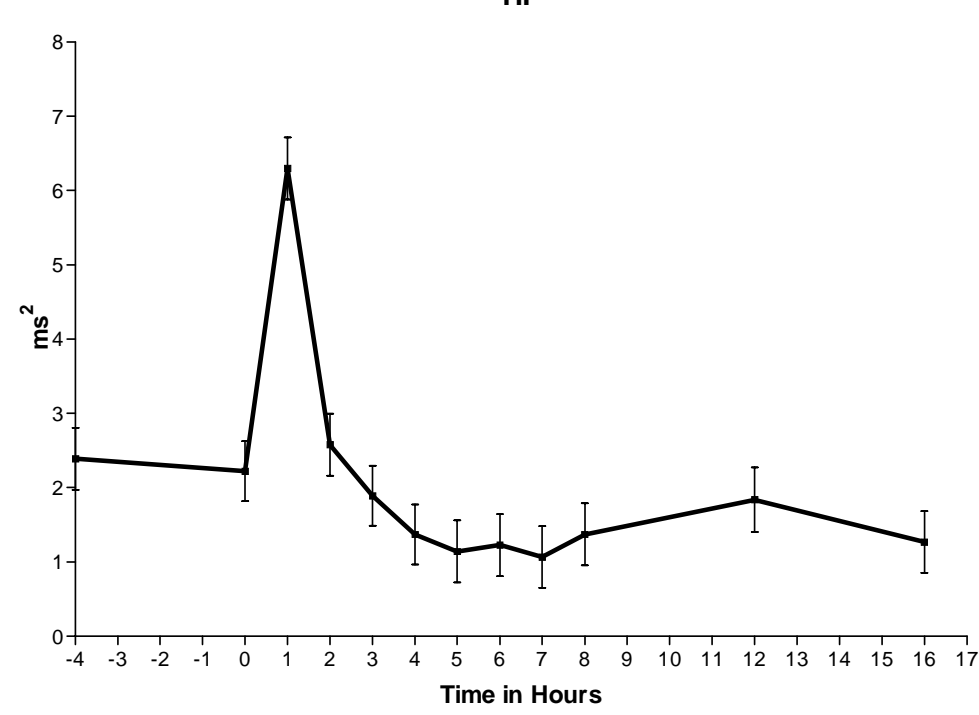

HF nu

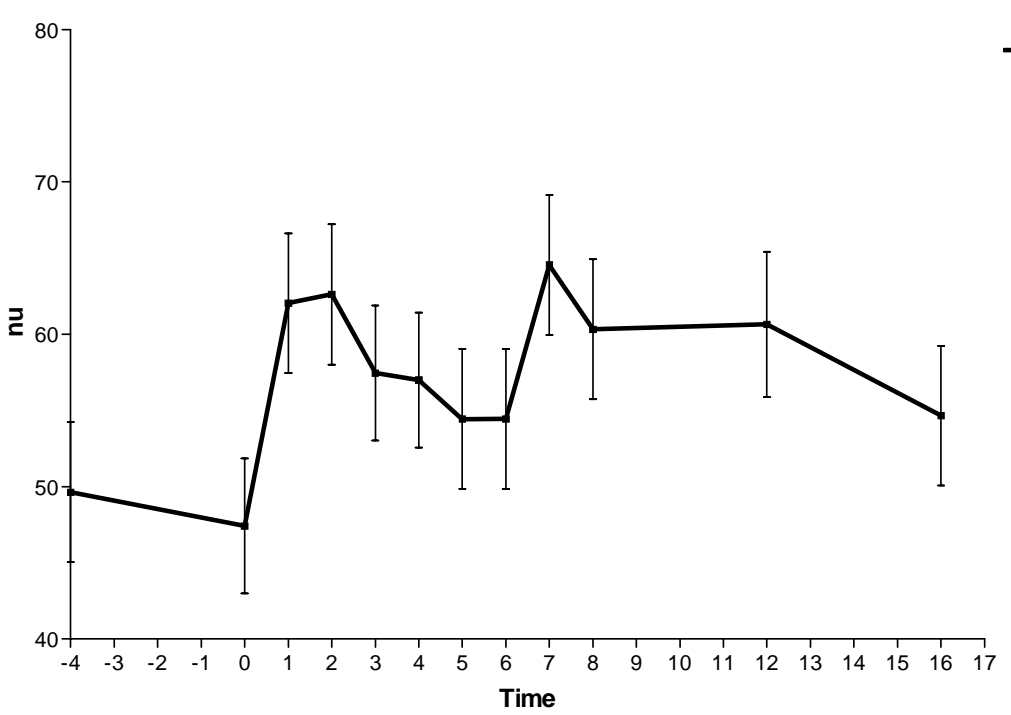

Figures 35, 36, 37.

Measurements of

parasympathetic tone in both the time (RMSDD) and frequency domain ( $H F$ and $H F n u$ ).

RMSDD and HF demonstrate an initial increase in parasympathetic tone that drops below control values at 4 hours after LPS (given at time 0). HF nu which normalizes for the decrease in total HRV suggests an initial increase in parasympathetic activity that remains moderately elevated throughout the experimental period. Time is in hours before or after LPS 
LF

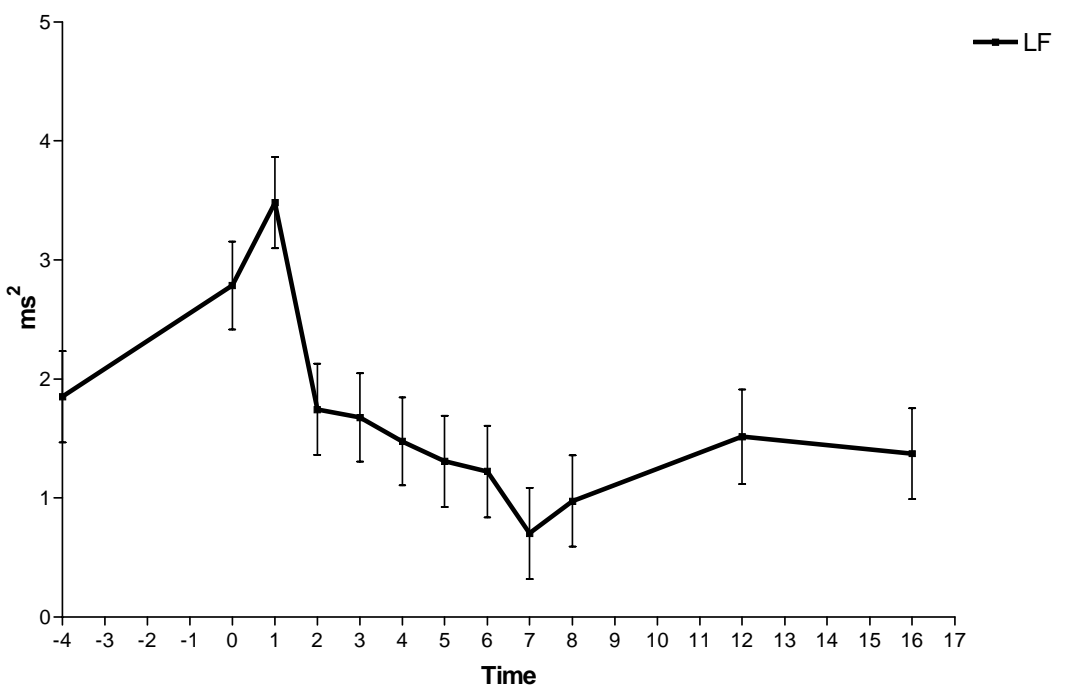

Figures 38, 39, 40. Sympathetic component of HRV as measured by $L F, L F$ nu and $L F / H F$. $L F$ shows a significant decrease in sympathetic input $(p<0.05)$ from 2 hours onward. There are no significant differences in the autonomic input as measured by $L F$ nu and the LF/HF ratio. Time is hour before or after LPS adminstration at time 0 .

LF nu

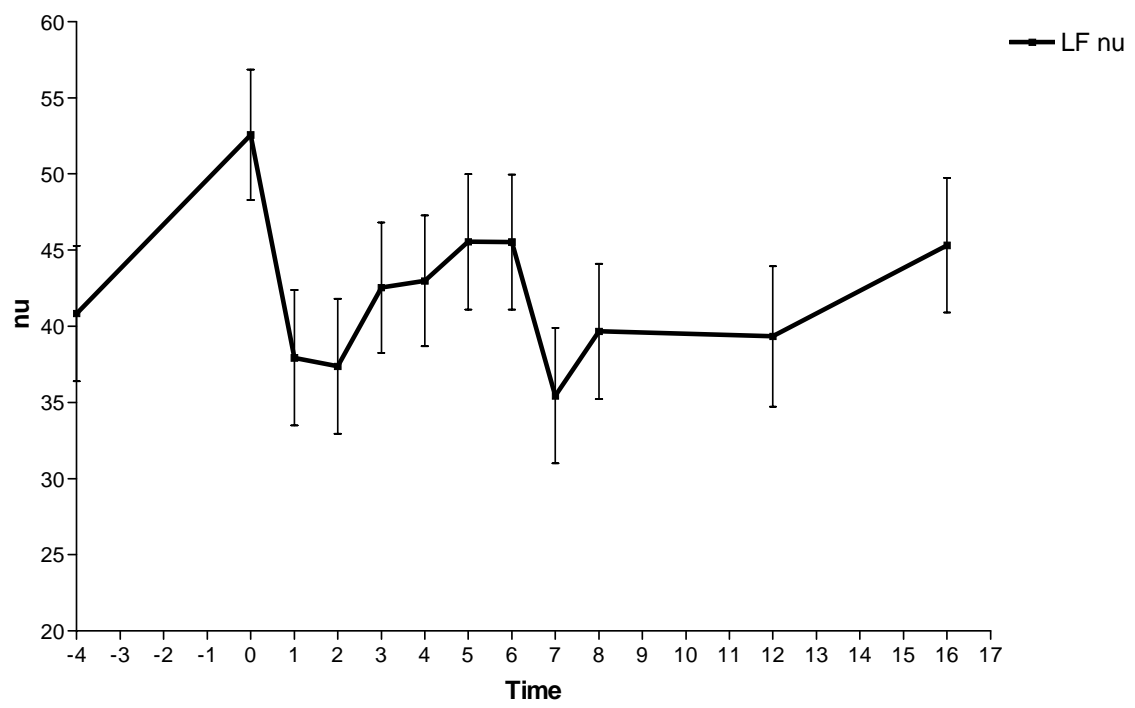

LF/HF

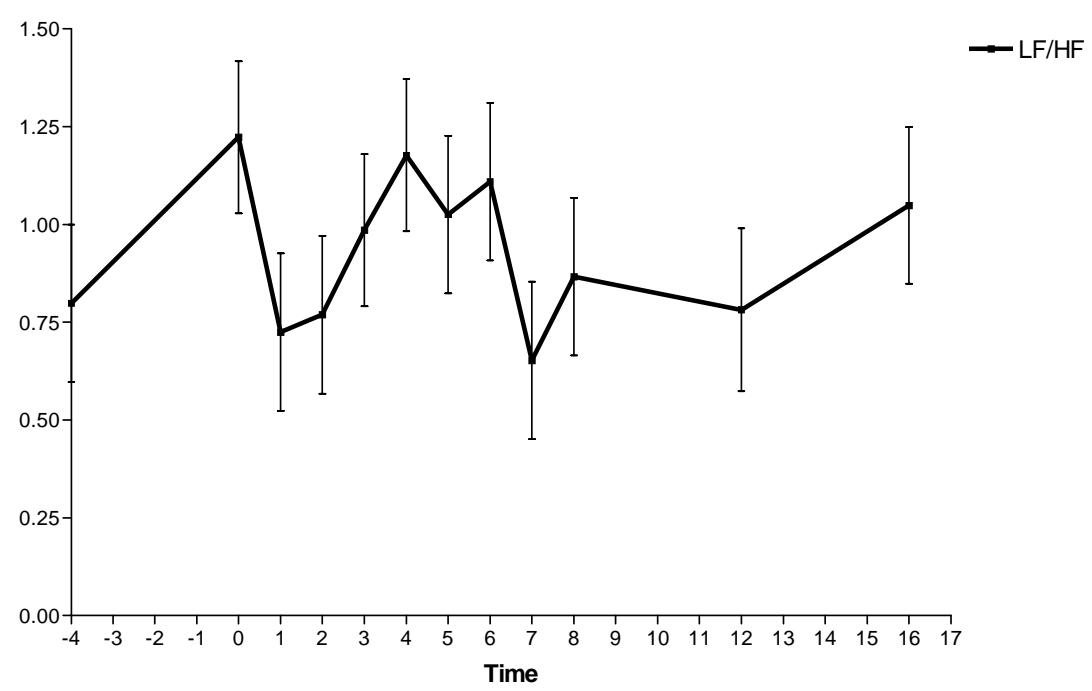




\section{Relationship between inotropic and chronotropic changes}

The findings that there is an elevation of in vivo IHR and in vitro HR in sepsis, and simultaneously that there was a lack of contractile dysfunction is at odds with the literature. As previously noted there is no standardized model for the study of endotoxemia induced cardiac dysfunction. Most of the studies that have observed cardiac dysfunction in the rat have done so after a relatively short duration, approximately four hours after endotoxin administration, or after continuous exposure to endotoxin or bacteria. In both of these instances the cardiac dysfunction could be relatively acute in nature. Our model consisting of endotoxin administration as a single bolus followed by the determination of cardiac function 16-20 hours later is unique. To examine the time course of HR and contractile changes following a $5 \mathrm{mg} / \mathrm{kg}$ dose of endotoxin the following experiment was conducted. Rats were randomized to receive either LPS or saline (NS). Equal numbers of animals from each group were sacrificed four hours or 16-20 hours after endotoxin administration and in vitro cardiac function was examined. Unlike the previous experiments, after baseline cardiac function was determined, the hearts were paced at various HRs. The reason for this was twofold: 1) to control for the effect of HR experimentally, rather than statistically; and 2) to examine the effect of increasing HR on cardiac function at physiologic HRs.

In these rats the in vivo HR in endotoxin treated animals was elevated above control at both 4 and 16 hours, the in vitro HR was elevated only at 16-20 hours (figure 41). When examining the difference on a percentage basis the greater elevation of in vivo HR occurs at 16-20 hours and there is the same percentage increase in vitro (table 13). 


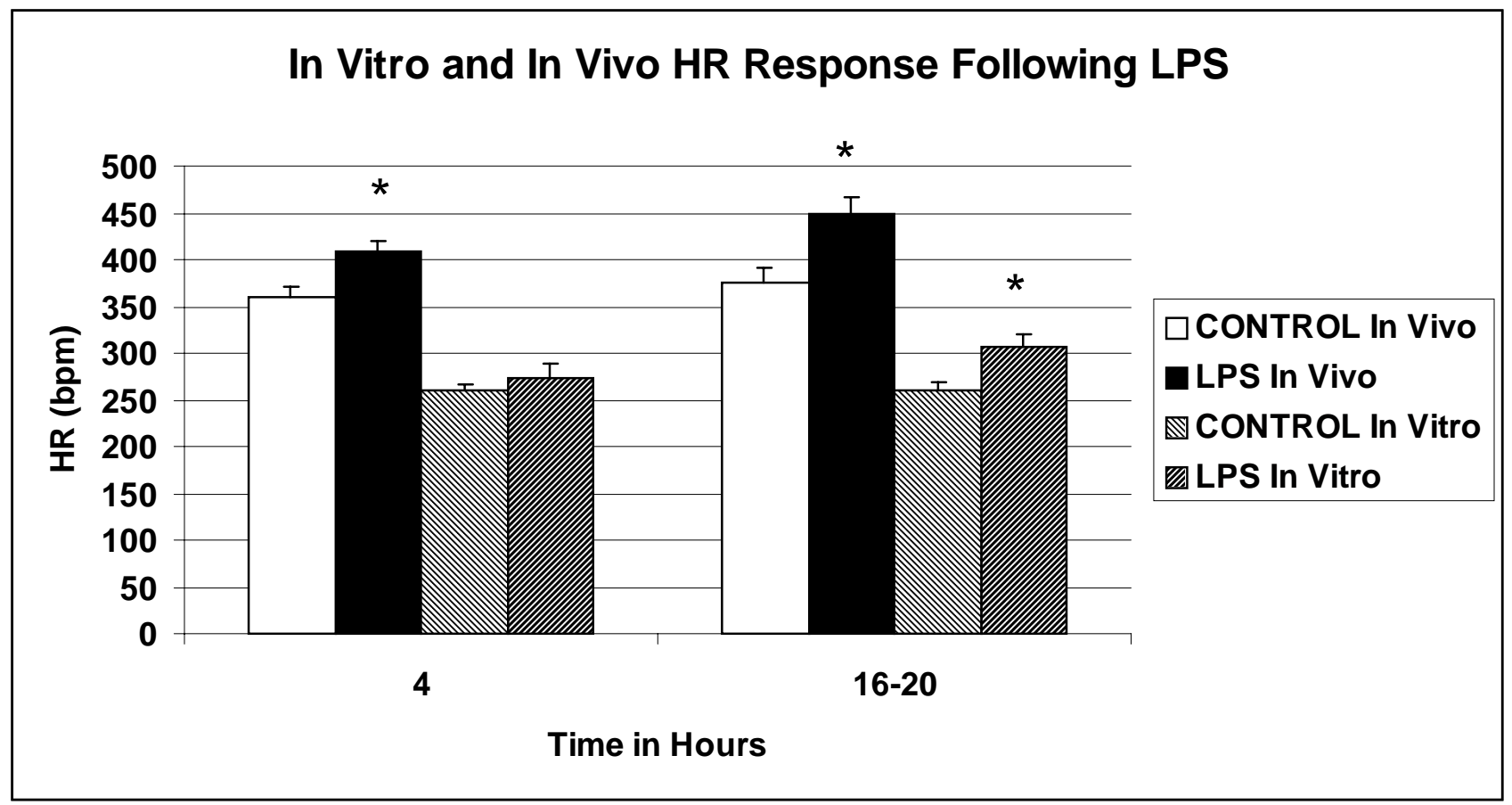

Figure 41. Heart rate (HR) in vivo and in vitro both four and 16-20 hours following endotoxin (LPS, $5 \mathrm{mg} / \mathrm{kg}$ i.p.) or saline (CONTROL) in vivo. * denotes significantly different than control $p<0.05$ ( $n=7$ to 10 per group).

\begin{tabular}{|c|c|c|c|c|}
\hline & \multicolumn{2}{|c|}{4 HOURS } & \multicolumn{2}{c|}{16 HOURS } \\
\hline & In Vivo HR & In Vitro HR & In Vivo HR & In Vitro HR \\
\hline CONTROL & $360 \pm 12(10)$ & $259 \pm 8(10)$ & $375 \pm 17(7)$ & $259 \pm 15(7)$ \\
\hline LPS & $408 \pm 12(9)$ & $274 \pm 9(9)$ & $448 \pm 19(7)$ & $306 \pm 14(7)$ \\
\hline \% Difference & $13 \%$ & $6 \%$ & $19 \%$ & $18 \%$ \\
\hline & $\mathrm{p}=0.012$ & $\mathrm{p}=0.214$ & $\mathrm{p}=0.016$ & $\mathrm{p}=0.041$ \\
\hline
\end{tabular}

Table 13. Heart rate in beats for minute (HR) observed in vivo and in vitro both four and 1620 hours following endotoxin (LPS, $5 \mathrm{mg} / \mathrm{kg}$ i.p.) or saline (CONTROL) treatment in vivo. In vivo HR was recorded in anesthetized animals. Number in parenthesis is $n$ per treatment group.

These data demonstrate the consistent $(15-20 \%)$ elevation in both in vivo and in vitro HR at 16-20 hours after endotoxin administration. It further shows that the elevation in in vitro, and therefore intrinsic, HR is unlikely to be observed at four hours. 
The earlier experiments had demonstrated no contractile dysfunction at 16 hours, as measured by cardiac output and aortic dP/dt. In examining cardiac output, preload and afterload were controlled experimentally, and there was a statistical adjustment for HR, so this should have been a reliable measure of contractility. The maximum rate of ventricular pressure development ( $\mathrm{V} \mathrm{dP/dt}$ ), or the left ventricular developed pressure (LVDP), for a given preload and HR are recognized as better indices of contractile function. Based upon these measurements, significant contractile dysfunction was observed four hours after endotoxin exposure but this reduced capacity had resolved by 16-20 hours (figures 42-45)
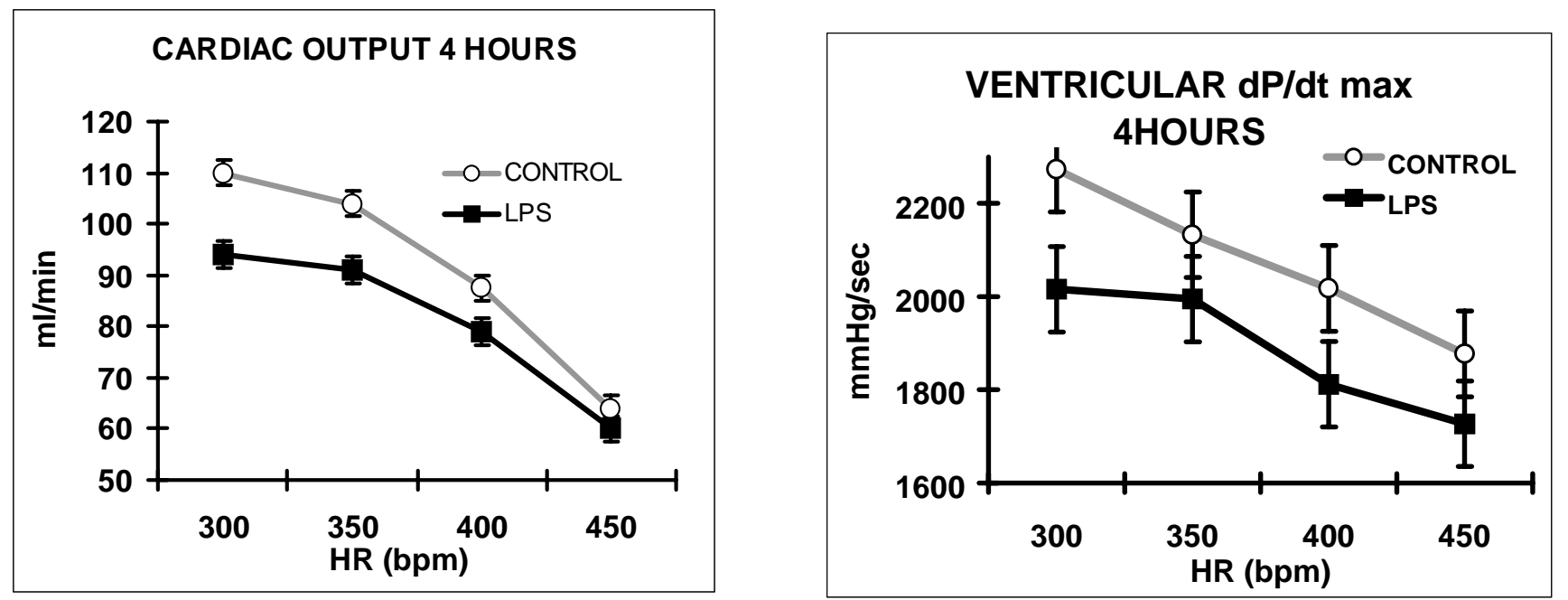

Figures 42 and 43. Cardiac output and left ventricular dP/dt max for increasing heart rates (HR) 4 hours after LPS $(5 \mathrm{mg} / \mathrm{kg})$ or saline (Control) administered in vivo. Cardiac output lines are significantly different $(p=0.03)$ as are $L V d P / d t(p=0.05)$ 

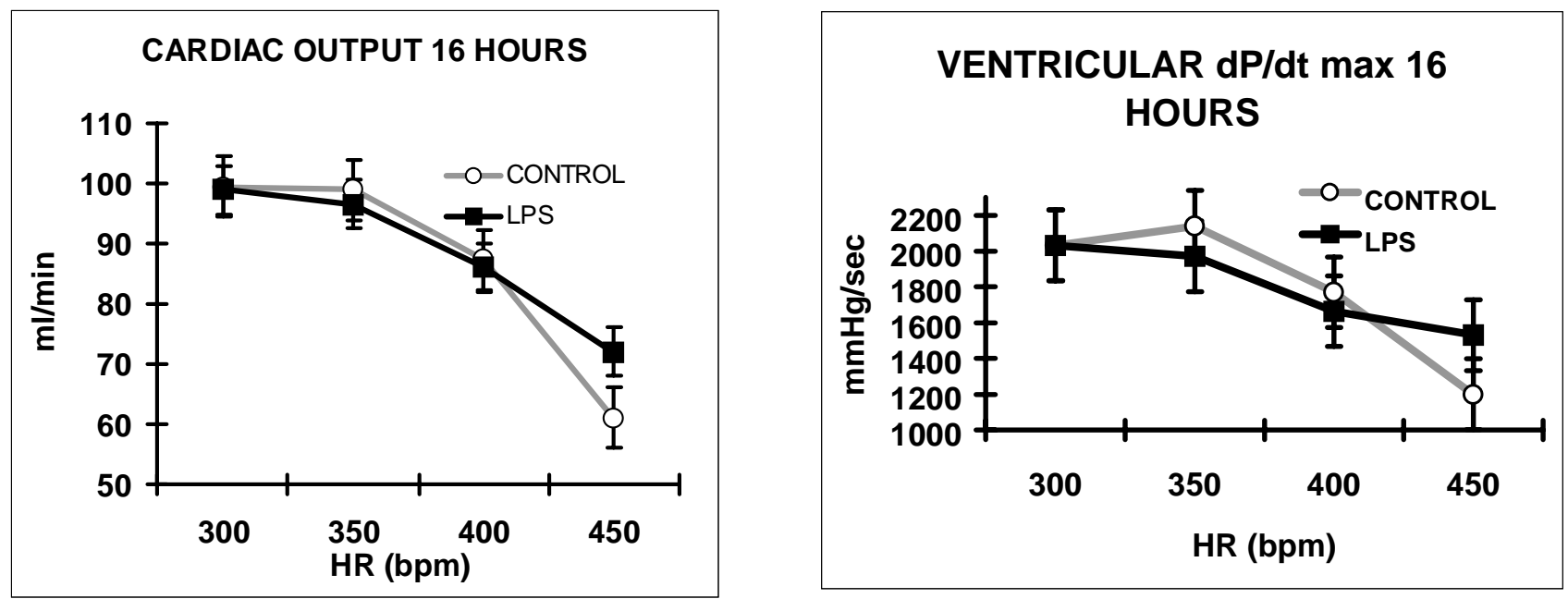

Figures 44 and 45. Cardiac output and left ventricular dP/dt max for increasing heart rates(HR) 16-20 hours after LPS (5 $\mathrm{mg} / \mathrm{kg})$ or saline (Control) administered in vivo.

Cardiac output lines are not significantly different $(p=0.96)$ nor are the $L V d P / d t$ lines $(p=$ 0.86)

It is not clear why the control values for $\mathrm{V} \mathrm{dP/dt}$ max are not equal at the two time points.

However, since the two experiments were conducted separately, this difference may be a

result of some variability in the apparatus from day to day. When both experiments, 4 and 16

hours after treatment, were analyzed together there is no significant difference between the

two time periods with respect to control animals. The observation of decreased contractility

at 4 hours and not at 16 to 20 hours was consistent regardless of the parameter used to

estimate contractility (table 14). 


\begin{tabular}{|c|c|c|c|c|}
\hline & \multicolumn{2}{|c|}{ 4 Hours } & \multicolumn{2}{c|}{16 to 20 Hours } \\
\hline & NS & LPS & NS & LPS \\
\hline Cardiac Output (ml/min) & $91.3 \pm 4.6$ & $81.1 \pm 5.3^{*}$ & $86.7 \pm 5.1$ & $88.5 \pm 4.0$ \\
\hline Stroke Volume (ml) & $0.256 \pm 0.01$ & $0.225 \pm 0.01^{*}$ & $0.242 \pm 0.01$ & $0.245 \pm 0.01$ \\
\hline Aortic dP/dt (mmHg/sec) & $802 \pm 60$ & $715 \pm 68$ & $910.7 \pm 128$ & $1091 \pm 117$ \\
\hline LV dP/dt (mmHg/sec) & $2076 \pm 132$ & $1887 \pm 132^{*}$ & $1756 \pm 171$ & $1799 \pm 156$ \\
\hline LVDP (mmHg) & $102 \pm 7.0$ & $95.3 \pm 7.0$ & $109 \pm 10$ & $109 \pm 9$ \\
\hline
\end{tabular}

Table 14. Contractile response at 4 and 16 to 20 hours after $5 \mathrm{mg} / \mathrm{kg}$ LPS i.p. Numbers represent the average response for $H R$ s of 300, 350, 400 and 450 determined by electrical pacing. $L V d P / d t$ and $L V D P$ are for a preload of $15 \mathrm{mmHg}$. * represents significantly different from control $p<0.05$.

Four hours after endotoxin administration there was a definitive decrease in contractility that had resolved by 16 hours. As can be seen in the above figures, increasing HR results in a negative slope for each of the parameters of contractility. Based upon the fit of these lines, an $18 \%$ increase in HR (the average value of the rise in intrinsic rate) at 16 hours would result in a $13 \%$ reduction in $\mathrm{CO}$, a $30 \%$ decrease in $\mathrm{SV}$, an $11 \%$ drop in $\mathrm{V} \mathrm{dP/dt}$ max, a $32 \%$ decline in A dP/dt max and a 17\% decrease in LVDP. Therefore, as discussed in the introduction, increasing HR through mechanisms outside of the autonomic nervous system, pacing in this case, results in a significant decrease in cardiac function. This directly relates IHR to cardiac performance and suggests that changes in IHR may directly alter cardiac function.

Using the reserpine pretreatment regimen that resulted in the abolition of the increase in IHR (ie. $1 \mathrm{mg} / \mathrm{kg} 32$ and 8 hours before endotoxin) we examined contractile function of animals sacrificed 4 hours after LPS. Similar to the four hour time course experiments 
described above, endotoxin resulted in a decrease in cardiac function. This decrease in contractile function was not altered, positively or negatively, by reserpine administration (table 15).

\begin{tabular}{|c|c|c|c|c|c|c|c|c|}
\hline & \multicolumn{3}{|c|}{ Effects of LPS Treatment } & \multicolumn{3}{c|}{ Effects of Reserpine Treatment by LPS Treatment } \\
\hline & NS & LPS & $\mathrm{p}$ & Sham-NS & Sham-LPS & Res-NS & Res-LPS & $\mathrm{p}$ \\
\hline $\mathrm{HR}(\mathrm{bpm})$ & $237 \pm 8$ & $274 \pm 8$ & 0.008 & $242 \pm 10$ & $268 \pm 13$ & $233 \pm 11$ & $279 \pm 11$ & 0.4 \\
\hline $\mathrm{CO}(\mathrm{ml} / \mathrm{min})$ & $104 \pm 7$ & $82 \pm 7$ & 0.036 & $104 \pm 9$ & $75 \pm 11$ & $104 \pm 9$ & $88 \pm 9$ & 0.5 \\
\hline $\mathrm{CF}(\mathrm{ml} / \mathrm{min})$ & $20.0 \pm 1.6$ & $16.5 \pm 1.7$ & 0.15 & $19.4 \pm 2.3$ & $17.4 \pm 2.6$ & $21.2 \pm 2.3$ & $16.1 \pm 2.3$ & 0.5 \\
\hline $\mathrm{A} \mathrm{dP} / \mathrm{dt}$ & $1101 \pm 82$ & $757 \pm 89$ & 0.03 & $1279 \pm 116$ & $868 \pm 134$ & $973 \pm 116$ & $800 \pm 116$ & 0.34 \\
\hline $\mathrm{CO} \times \mathrm{PSP}$ & $7432 \pm 535$ & $5134 \pm 578$ & 0.01 & $7877 \pm 757$ & $5112 \pm 874$ & $7702 \pm 757$ & $5969 \pm 757$ & 0.53 \\
\hline
\end{tabular}

Table 15. In vitro response of contractile parameters to endotoxin and the combined effects of reserpine and endotoxin administered in vivo. Reserpine (Res) or sham vehicle (Sham) was administered in vivo (1 $\mathrm{mg} / \mathrm{kg}$ i.p.) 8 and 32 hours prior to LPS or saline (NS) and animals were sacrificed 4 hours after LPS or NS. The first two columns show the effect of NS or LPS across treatments. The p value in the $3^{\text {rd }}$ column refers to the ANOVA with regard to this treatment. The following four columns show the interaction between the two treatments. The $p$ value in the last column refers to the interaction between the two treatments. Heart rate $(H R)$ is in beats per minute (bpm). Cardiac output $(\mathrm{CO})$ is milliliters per minute $(\mathrm{ml} / \mathrm{min})$ as is coronary flow $(\mathrm{CF})$. Aortic change in pressure of change in time $(\mathrm{A} d \mathrm{dP} / \mathrm{dt})$ is recorded as $\mathrm{mmHg} / \mathrm{sec}$. Cardiac output times peak systolic pressure (CO $x$ PSP) is recorded as $\mathrm{ml} / \mathrm{min} \bullet \mathrm{mmHg}$. An examination of the ANOVA terms and interaction terms shows that all of the variability lies within the LPS treatment and that LPS has a negative effect on the contractile parameters and increases in vitro $H R, n=7$ to 8 animals per group.

As seen in table 15, in this experiment, unlike the previous experiments, the in vitro HR was significantly elevated at 4 hours. The effect on heart rate was entirely within the LPS treatment. The graph below demonstrates that treatment with reserpine did not abolish this difference in heart rate (figure 46). 


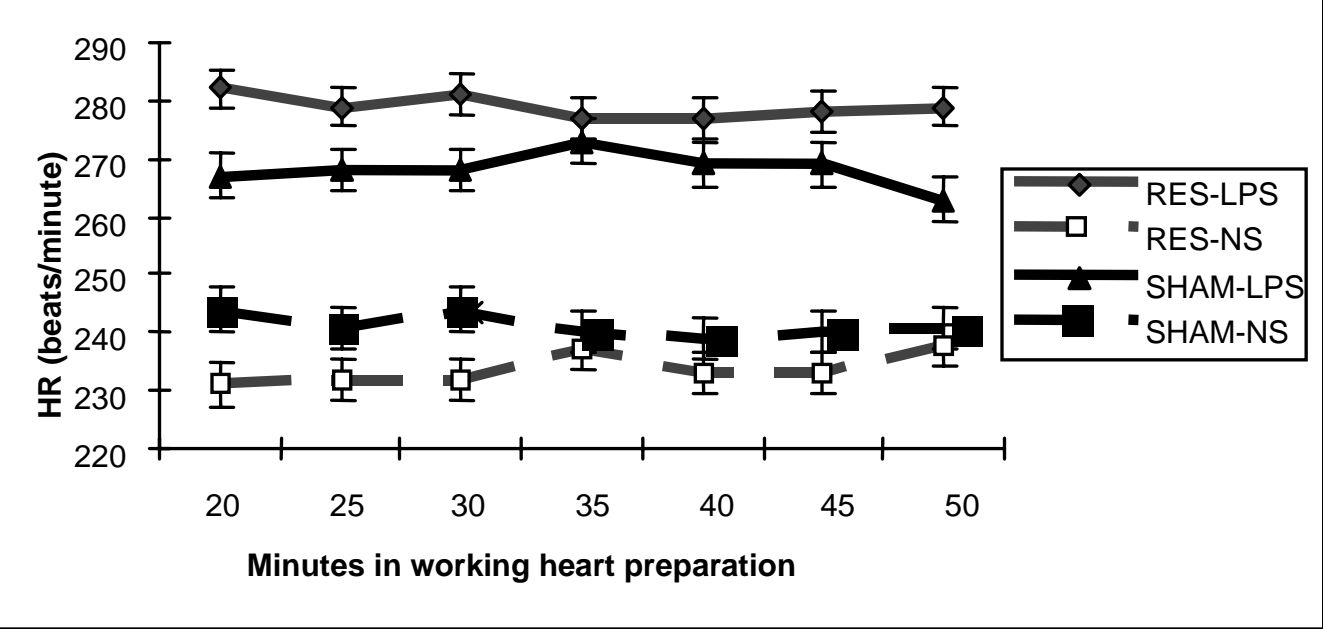

Figure 46. In vitro HR response four hours after endotoxin (LPS, $5 \mathrm{mg} / \mathrm{kg})$ or NS treatment in vivo. Animals had been pretreated with reserpine (RES $1 \mathrm{mg} / \mathrm{kg}$ ) or sham vehicle 32 and 8 hours prior to LPS.

It is likely that this elevation in intrinsic heart rate was observed at four hours after endotoxin treatment because the analysis of individual treatments and interaction between treatments effectively doubles the $\mathrm{n}$ to $15-16$ animals per group when compared to experiments with only $7-8$ animals per group. In other words, in the previous experiments there was a trend toward the elevation of intrinsic heart rate a 4 hours but the sample size was insufficient to have adequate statistical power to detect it. This suggests that the intrinsic heart rate may begin to become elevated around 4 hours. It is more difficult to explain why reserpine did not prevent the elevation of in vitro HR in this experiment. One possibility is that LPS induces an increase in the regulation of intrinsic heart rate which requires the presence of catecholamines to become manifest. If at four hours there was still incomplete depletion of catecholamines by reserpine the in vitro HR would be elevated but by 16 hours with depletion of catecholamines complete the reserpine-LPS heart rate has returned to control. As will be discussed below, in this scenario the order and mechanism by which the $\beta A R$ and LPS and/or cytokines generate the elevation in IHR is unclear. What is clear is that both mechanisms are required for the development of the persistent elevation in HR. 


\section{DISCUSSION}

Tachycardia is a ubiquitous finding in sepsis and endotoxemia. This increase in heart rate is largely viewed to be a compensatory response of the autonomic nervous system to hypotension and tissue hypoxia. The tachycardia of sepsis, however, develops slowly in comparison to changes in blood pressure, lasts longer than the hypotension, and even continues after the patient or animal is volume resuscitated. These findings suggest that the tachycardia of sepsis is not baroreceptor mediated (Qi et. al., 1991; Yu et. al., 1997; Zhou et. al., 1991). Certainly, chemoreceptors in the aortic and carotid bodies, as well as those in the medulla, which sense hypoxia or hypercarbia could also play a role in increasing heart rate (Levick, 1995). It is interesting to note that the tachycardia of sepsis is observed and even increases, after plasma catecholamines reach their apex and plateau or decline (Jones and Romano, 1989). When attempting to determine the origins of this tachycardia an even greater paradox is that many investigators have demonstrated that the decrease in myocardial contractile performance in sepsis is, in part, related to dysfunction or desensitization of the ß1-adrenoceptor (Bensard et. al., 1994; Jones and Romano, 1990; Werdan et. al., 1991). If this is the case, and the same class of $\beta$ ARs that mediate inotropy also mediate chronotropy, the question arises, why is tachycardia observed rather than bradycardia? There has been reported evidence to suggest that the tachycardia is non-autonomic in origin. It has been demonstrated using analysis of heart rate variability that during the tachycardia of endotoxemia there is a decrease or absence of sympathetic input to the heart (Garrard et. al., 1993; Godin et. al., 1996; Goldstein et. al., 1995). Other investigators have suggested that calcitonin gene related peptide may play a role in the cardiovascular derangements of sepsis 
including tachycardia (Arden et. al., 1994; Huttemeier et. al., 1993). All of these findings allude to the possibility that the tachycardia of sepsis may be intrinsic to the heart itself.

The fact that the heartbeat is myogenic, or intrinisic, in origin is no longer a matter of debate, nor is the central role that the autonomic nervous system plays in regulating the rate of the heartbeat. What is less recognized is that, in certain situations, the intrinsic rate at which the heart beats can be altered. The IHR has been found to be decreased with age, and exercise training has been shown to lower the IHR in both animals and humans. At this time there is no widely recognized state in which the IHR is increased. A pharmacologic method of determining the intrinsic heart rate was first proposed in 1966 (Jose et. al., 1966) and has been used extensively since that time. Obviously, the excision and perfusion of the isolated heart also provides a means of studying the intrinsic heart rate.

Given these two observations, one, that there are incongruencies in the literature with regard to the mechanism of tachycardia in sepsis, and secondly that there is a predetermined intrinsic heart rate that can be altered in certain physiologic conditions, the following hypothesis was formed: An elevation in the intrinsic heart rate contributes to the tachycardia observed following the administration of endotoxin. The research conducted to test this hypothesis sought to answer three questions: (1) Is there an elevation of the intrinsic heart rate in sepsis? (2) How is the elevation of IHR initiated and maintained? (3) Is there a differentiation between the inotropic and chronotropic responses to sepsis? The results clearly answer the first and third questions in the affirmative. With regard to the second question, the initiation and maintenance of the elevation in IHR, the current findings suggest that there is a requirement for both $\beta$ AR stimulation and LPS or another factor induced by it. The cellular mechanism by which this occurs remains to be further defined. 
In order to characterize the model of endotoxemia used, a single intraperitoneal injection of endotoxin $(5 \mathrm{mg} / \mathrm{kg}$ ) followed by the observation of cardiac function 16-20 hours later, we first examined in vivo HR and blood pressure changes over a 24 hour time course. In this model, endotoxin caused no significant changes in blood pressure. This is not an uncommon finding in the literature particularly with lower doses, endotoxin administered in ways other than an intravenous bolus, and in cecal ligation and puncture models (Fish et. al., 1985; Lubbe et. al., 1993; Palsson et. al., 1988; Shepherd et. al., 1987; Smith et. al., 1986). Additionally, the in vivo heart rate did not become elevated until four to five hours after the administration of LPS and remained elevated thereafter through the end of the monitoring period. The experiment examining heart rate variability had similar findings. This delay in the development the tachycardia is also not surprising. There is a lag time after the i.p. injection of endotoxin before endotoxemia develops. With a $5 \mathrm{mg} / \mathrm{kg}$ dose of endotoxin, plasma endotoxin levels do not plateau until two hours after administration and remain elevated for five hours (Baharami et. al., 1993), cytokine production takes additional time, with a peak of TNF at 30 minutes to one hour and IL-1 and IL-6 peaks between two and four hours following exposure to LPS (Ayala et. al., 1992; Dunn, 1994; Kunkel et. al., 1994). Because of this delay a slowly developing tachycardia is a common observation (Jones and Romano, 1989). In the current study, the animals were clearly ill by four to six hours based upon their toxic appearance. At sixteen hours the endotoxin treated animals were observed to have lost $4.8 \%$ of their body weight. This finding, the loss of body weight, provided a consistent means of assessing the effectiveness of the endotoxin administration.

In characterizing the model we were particularly interested in the later time periods. Although it is well documented that the administration of endotoxin results in myocardial 
dysfunction by four hours, it was not until after this that the heart rate became consistently elevated. Many investigators utilize the relatively short time period of four hours, likely for experimental convenience, when studying the effects of endotoxin on contractile function. Our research suggests that at this time point it is possible that changes in IHR have not occurred or are just being initiated. Other investigators, who use longer time courses to study endotoxemia or sepsis, often do so in models that provide for the continual exposure of the animal to endotoxin. While these models, such as cecal ligation and puncture, osmotic minipumps, infected clots, and subcutaneous abscesses, are better duplicates of the human septic process, they do not separate the long term effects of endotoxin and the adaptive responses of the host from the acute effects associated with repeated exposure. This could be an important distinction as one of the prevailing hypotheses in sepsis research at this time is that a derangement in the balance of initially protective host responses to endotoxin causes much of the morbidity and mortality associated with sepsis (Bone, 1996). Along these lines, it has been our observation that those investigators who have found an elevation of the in vitro HR have employed models that were longer in duration (Barker et. al., 1990; Fish et. al., 1985; Muniz et. al., 1991, Smith et. al., 1986). Because an elevation in the IHR was of specific interest, and we felt it to be a slowly developing effect of endotoxin, a model that observed cardiac function 16-20 hours after a single bolus LPS injection was used.

Pharmacologic antagonism of both the sympathetic and parasympathetic input to the heart in vivo provides an estimate of the IHR. Furthermore, by determining the individual effects of atropine and propranolol the relative inputs of each system to the heart rate can be determined. In this rat model of endotoxemia we found the in vivo HR to be increased by $19 \%$ over controls. Interestingly, the relative effects of the sympathetic and parasympathetic 
systems were equal in both the control and septic animals. The IHR of the septic animals was $21 \%$ greater than control animals. As confirmation of these findings we also observed a $23 \%$ difference in the heart rates of control and endotoxin treated animals when exposed to the ganglionic blocker hexamethonium. These experiments confirmed the hypothesis that there was an elevation of the IHR in sepsis and further suggested that the entire increase in HR, on a percent basis, was due to the elevation of IHR rather than a modification of neural regulation. In agreement with this, the studies of heart rate variability did not show a significant alteration in the relative levels of autonomic input to the heart, despite a significant decrease in total variability.

The possibility remained at this point that the difference in heart rate observed after the administration of these drugs was related to humoral substances that were either products of the autonomic nervous system, or were not "classic" autonomic neurotransmitters such as CGRP or other neuropeptides. Excision of the hearts and perfusion in oxygenated buffer should remove the heart from the influence of all humoral substances. When this experiment was conducted we consistently observed a $15-20 \%$ higher in vitro HR of the rats treated in vivo with endotoxin 16-20 hours earlier. The heart rate remained elevated for at least one hour in this preparation. This percent increase in in vitro HR closely agrees with what others have found (Fish et. al., 1985; Romanosky et. al., 1986; Smith et. al., 1986) and is remarkably consistent with the in vivo changes that were seen in this study. These observations exclude the possibility that the elevation in IHR requires the continuous presence of another "hormone" like CGRP, adrenomedullin or angiotensin.

It is equally important to rule out the possibility that the elevation in IHR is caused by any of the other previously described mechanisms delineated in the introduction. The 
Bainbridge or Anrep effect can be excluded for several reasons. The preparation used is an isolated working left heart preparation in which buffer distends the left atrium and is pumped into the ventricle and then ejected. The left atrial pressure is constant at $15 \mathrm{~cm}$ in both control and endotoxin treated hearts. If the left atrium is more compliant in septic animals, it is possible that it is stretched further (contains greater volume for a given pressure) than the atrium of control animals. The Bainbridge response is elicited by the act of stretching not the degree of stretch. With constant pressure, although there may be increased distension of compliant atria, there is no mechanism by which the degree of stretch is altered and so the Bainbridge response would not occur. More importantly, the Bainbridge response can be excluded because this response is not present in the left atrium and the right atrium is not perfused in this preparation (Jensen, 1971).

Alteration in temperature is another mechanism described to alter the intrinsic heart rate (Knowlton and Starling, 1912; Walsh, 1969). Increases in temperature result in increases in HR directed by mechanisms outside of the autonomic nervous system. In examining the core temperature of ten rats that had been treated with LPS or saline we did observe a significant difference. LPS treated animals had a mean temperature of $37 \pm 0.12^{\circ}$ $\mathrm{C}$ versus $35.9 \pm 0.12^{\circ} \mathrm{C}$ for control animals. These observations were made in anesthetized animals and were anticipated, as LPS is a pyrogen. It is unlikely that this small difference in temperature could have accounted for the difference in intrinsic heart rate in vivo (Walsh, 1969). The question is moot with regard to the observed difference in in vitro heart rates because the buffer and apparatus were heated to the same constant temperature for both groups of animals. Changes in osmolality and ion concentrations occur in sepsis and can effect heart rate. While it is possible that these factors could have played a role in producing 
the observed difference in in vivo intrinsic heart rate, they can not account the in vitro observations because the large volume of physiologic buffer with which the hearts were perfused would abolish any differences in ion concentration. This is not to say, however, that alterations in ion channel conductance or membrane permeability are not playing a role in the elevation of IHR. In fact a decrease in the resting membrane potential (RMP) of rat ventricular myocytes from $-74 \mathrm{mV}$ to $-68 \mathrm{mV} 18$ hours after cecal ligation and puncture has been demonstrated (Wu et. al., 1993). This type of change in RMP, if also occurring in pacemaking cells, would decrease the time to depolarization and provide a potential mechanism for the increase in IHR.

Although alluded to elsewhere in this work, a detailed discussion of the possible reasons why we have observed an elevation in the IHR in sepsis where others have not is appropriate. The first point is that others have made this observation (Barker et. al., 1990; Fish et. al., 1985; Muniz and McDonough, 1991; Smith et. al., 1986). Fish and co-workers found this observation so unusual that they included the following in their discussion:

"The reason for this in vitro tachycardia after $6 \mathrm{hr}$ of endotoxin infusion is unknown, but cannot be attributed to systematic experimental error. The rates were appreciably higher than routinely recorded in this laboratory, and were observed under the same operating conditions as controls (i.e., the same apparatus and buffer; control and endotoxin hearts on the same day)." (Fish et. al., 1985)

Other investigators have seen differences in rate but did not have sufficient experimental power to show statistical differences (Romanosky et. al., 1986). Furthermore, because most experiments were designed to study contractile rather than chronotropic changes, and therefore, hearts were subjected to electrical pacing, which would mask any differences in HR that may have existed. Additionally, our model is somewhat different in that it documents the prolonged effects of a single dose of endotoxin. Lastly, the sepsis literature 
has repeatedly demonstrated a tachycardia simultaneous with autonomic dysfunction or $\beta \mathrm{AR}$ dysfunction which strongly suggest an elevation of IHR.

There is evidence in the experimental results presented for two factors, besides LPS, which significantly altered the IHR. The first is the relationship between weight and HR that indicated that the IHR declined by 0.2 beats per minute with each gram increase in body weight. The effect of increasing heart weight on IHR has been reported (Harri and Kuusela, 1982). Because rats grow at a constant rate it is likely in this instance that the increase in weight served simply as a marker for increasing age which has also been demonstrated to result in a decrease in IHR in the rat (Kreider et. al., 1984). The other effect, that of anesthetic, is less well described. Pentobarbital was found to decrease the IHR in both LPS and control animals by $15 \%$ when compared to Telazol. While it has been noted that pentobarbital can exacerbate endotoxin induced myocardial contractile dysfunction in rats (Hoque et. al., 1996), its effects on HR are less clear. Some investigators have reported that pentobarbital anesthesia induces an increase in in vitro $\mathrm{HR}$ and improvement of in vitro myocardial performance over decapitation (Rubanyi and Kovach, 1980; Sage et. al., 1985) others have found the opposite (Segal et. al., 1990). No studies examining the effect of Telazol on in vitro cardiac function in rats have been found to date. In large animal studies the drug has been shown to have minimal effect on in vivo cardiac performance (Lin et. al., 1991). It is especially important to note, however, that neither of these observations affected our experimental findings, as the effects were constant across both the LPS and control treatment groups regardless of the anesthetic employed.

The second question which the experiments sought to answer, is related to the mechanism of induction and maintenance of the increase in heart rate. When the 
catecholamine content of the rat was depleted by pretreatment with reserpine prior to endotoxin administration the elevation of the IHR was abolished. This effect could be explained via several mechanisms. One obvious possibility is that catecholamines are required to initiate the elevation in IHR. Catecholamines, are, however, clearly not required for the ongoing maintenance of the increased IHR as evidenced by the failure of propranolol to abolish the elevation in IHR in vivo or in vitro when administered after endotoxin. Alternatively, reserpine is somewhat nonspecific in its action and also depletes the nerve terminals of other neurotransmitters, notably 5-hydroxytryptamine (5-HT), which has been variably found to cause bradycardia and tachycardia (Dabire et. al., 1992; Cherqui et. al., 1988). Another possibility is that reserpine was toxic to the myocardium and a component of this toxicity affected HR. The toxicities of reserpine are well known (Casteels and Login, 1983; Wilcken et. al., 1967) and, although unlikely to occur at the doses used in these experiments, this remained a plausible hypothesis. To confirm that the effects which reserpine abolished are mediated by catecholamines, and more specifically through the $\beta \mathrm{AR}$, studies were conducted in which this receptor was antagonized prior to and during the induction of endotoxemia. Bromoacetyl alprenolol menthane (BAAM), an irreversible $\beta A R$ antagonist, administered prior to and during the septic insult also prevented the increase in IHR. This observation extended the findings of the experiments with reserpine to conclude that the elevation in IHR observed in sepsis was mediated via catecholamines acting at the $\beta A R$. It is noteworthy that when the hearts of the animals treated with BAAM were excised at 16-20 hours and used to construct dose response curves to isoproterenol the $\beta$ AR function appeared to be normal. 
The experiments in which there is an elevation of the in vitro heart rate at four hours after endotoxin administration following reserpine pretreatment but not at 16 hours makes the interpretation of these findings somewhat more challenging. It is known that rats are more resistant to the depleting action of reserpine than other small mammals such as the guinea pig (Rice et. al., 1987). To fully deplete the animal of catecholamines, particularly the adrenal medulla, often requires several administrations of relatively large doses of reserpine. It is entirely plausible that these animals were not fully depleted of catecholamines at the time of LPS administration and this remaining small releasable pool of catecholamines could provide the basis for the intriguing observation. This would further suggest that LPS is required to initiate the change in IHR but that $\beta$ AR stimulation was required to maintain the effect. If this were the case then the experiments conducted to examine the decay of the increased heart rate should have demonstrated different decay rates for LPS treated animals regardless of whether they had been pretreated with reserpine or not. In this experiment no significant differences were observed between the maximal heart rates or finishing heart rates. The experiment is somewhat inconclusive in that statistical differences were not observed in the basal rates of the isolated atria and that none of the heart rates had returned to basal by one hour after washout. Additionally the Sham-NS decrease in heart rate was steeper than the other three groups. There was, however, no difference between the reserpine-NS and reserpine-LPS groups. On the basis of this experiment the hypothesis that both effects ( $\beta$ AR stimulation and LPS) are required early in the septic process to initiate the change in IHR can not be excluded.

From the experiments of others, and our own observations, it is known that prolonged stimulation of the $\beta$ AR alone is not sufficient to elevate IHR (Matthews et. al., 1996; 
Nylander et. al., 1984). The current study demonstrated that activation of the $\beta$ AR is crucial in eliciting the elevation in IHR after LPS. Therefore, it is logical to conclude that the elevation of IHR is caused by a cellular event that requires both LPS, or the cytokines it induces, and stimulation of the $\beta \mathrm{AR}$. When examining the time course over which the elevation of IHR occurs there are several observations of note. The first is that the increase in in vivo HR is not observed before four hours after LPS suggesting that there is a factor involved which requires time to be produced or induced. The second is that in vivo HR becomes elevated, and stays elevated, in the absence of substantive differences in blood pressure suggesting the lack of involvement of baroreceptors and other reflexes. The third observation is that the in vitro HR remains elevated and constant for at least one hour following extirpation. The relatively long time course of all of these events points more towards an alteration in protein synthesis than protein function. Figure 47 illustrates the mechanisms by which $\beta$ AR stimulation increases heart rate that have currently been proposed. A change in protein synthesis, including an increase in synthesis, a decrease in synthesis or altered synthesis, of any one or more of the proteins in this pathway could alter the intrinsic heart rate. 


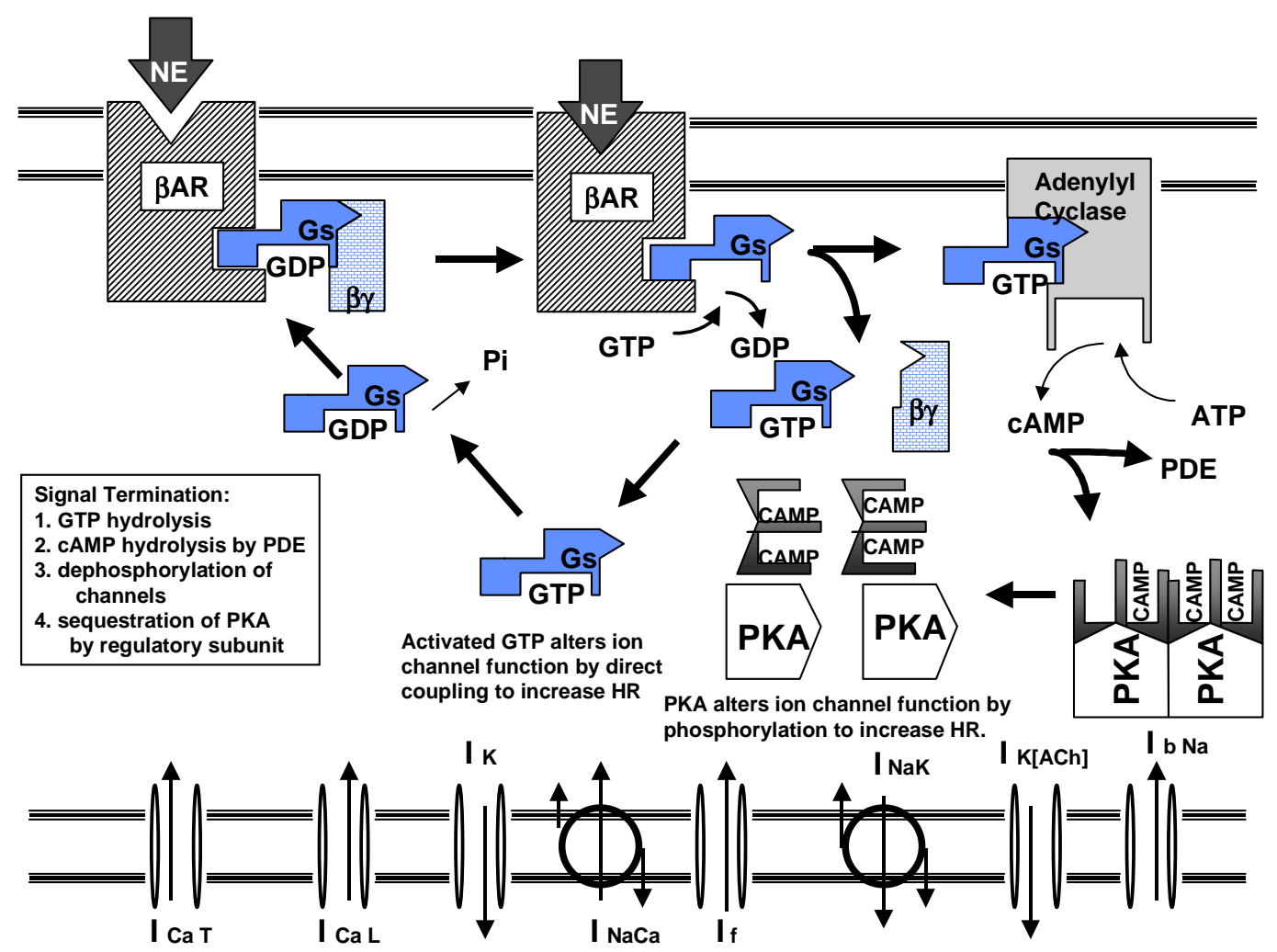

Ion channel changes that could increase heart rate:

1. Increase conductance or open probability of I Ca T, I Ca L, I f, I b Na.

2. Decrease conductance or open probability of I k or I k[Ach].

3. Depolarize cell membrane by increasing the activity of I $\mathrm{NaCa}$ or decreasing the activity of I NaK.

Figure 47. Normal signal transduction process used to increase HR. Norepinephrine (NE) stimulates the $\beta A R$. Gs is activated and does one of two things, it activates (or inactivates) channels and activates adenylyl cyclase. Four cAMP are required to bind the regulatory subunits of PKA before the catalytic subunits are released these subunits can phosphorylate ion channels to alter heart rate. All of the described channels are shown (Campbell et. al., 1992). Both Gs and PKA have been shown to alter If to increase HR (Yatani et. al., 1990). PKA is known to alter potassium channels (decrease conductance, Huang et. al., 1994) and calcium channels (increase conductance, Haase, 1996).

PKA-C is the mechanism by which the $\beta A R$ is known to signal to the nucleus. It is also known that with increasing stimulation of adenylyl cyclase and PKA that PKA-C levels ultimately decrease (Boundy et. al, 1998; Garrel et. al, 1997). Additionally PKA-C has been demonstrated to be activated in the right atrium early in the septic process (Hsu et. al., 1997). 
In light of this, the amount of PKA in the right atrium at several time points after the administration of endotoxin was examined. PKA-C levels declined to a third of control levels by sixteen hours after endotoxin, suggesting that at some point prior to sixteen hours there is likely to have been significant activation of PKA-C. The finding further demonstrates that it is unlikely that ongoing $\beta$ AR stimulation is responsible for the elevation in IHR at sixteen hours. Measurement of PKA-C levels does not provide any information which regard to the activity of PKA-C or the time course, if any, over which message is sent to the nucleus. An examination of the levels of phosphorylated CREB demonstrated that there is signalling to the nucleus (presumably through PKA-C) as early as two hours after LPS and that this signal peaks at fours after LPS. Interestingly Phospho-CREB levels have returned to control levels by 16 hours, again suggesting a possible early message to the nucleus that may result in an elevation of IHR.

The following description provides an example of a potential mechanism underlying the elevation of IHR for illustrative purposes. Assume that in order to increase HR, PKA must phosphorylate a potassium channel thereby decreasing the hyperpolarization of the cell and permitting an increase in HR. A protein phosphatase would remove this phosphate group placed on the channel allowing the HR to return to normal. If endotoxin, in concert with $\beta$ AR stimulation were to decrease production of the phosphatase via a change in protein synthesis the signal created by the phosphorylated channel would continue and HR would remain elevated. However, in order for the HR to increase initially would require some threshold of stimulation of the $\beta$ AR resulting in PKA activation to cause a sufficient number of channel phosphorylations to observe the increase in IHR. With reserpine administration and depletion of catecholamines this threshold of activation may never be reached and the 
increase in IHR would not be observed. The administration of BAAM before endotoxin could prevent either the reduction of the phosphatase, and / or PKA activation, thereby preventing the increase in HR. There is some support for this proposed mechanism since inhibition of phosphatases 1 and $2 \mathrm{~A}$ has been shown to prolong by up to 4 hours the decrease in potassium channel current induced by cAMP in mammalian neurons (Ansanay et. al., 1995). In guinea pigs inhibition of PKA resulted in a lowering of the elevation of HR induced by $\beta$ AR stimulation, and inhibition of phosphatases 1 and $2 \mathrm{~A}$ augmented the response. Furthermore, HR appeared to be elevated in two manners, a slower response phosphorylation and a faster response (presumably G-protein mediated) that was not related to phosphorylation. This could suggest why there is not an alteration of the chronotropic concentration response curve to isoproterenol after LPS despite the presence of decreased levels of PKA $-\mathrm{C}$. Interestingly these effects of $\beta A R$ activation did not extend to inotropism (Gotoh, 1995).

One consideration this proposed scheme addresses, that is not addressed in the currently available results, is the site at which the signal produced by endotoxin, or cytokines, and $\beta \mathrm{AR}$ stimulation interact. Is it at the level of altering protein transcription, or does one signal modify transcriptional events and the other serve as the reinforcement to perpetuate the change? One way to examine this question would be to administer isoproterenol to endotoxin exposed animals pretreated with reserpine. Presumably the alteration of, in this hypothesis, phosphatase production has occurred but PKA activity has not reached the required threshold for the change to be observed. Isoproterenol administered anytime after four hours would be predicted to cause sufficient PKA activation to result in a lasting increase in the IHR. Although the results are somewhat inconclusive because none of 
the heart rates returned to baseline, the results of the experiment exposing isolated right atria to isoproterenol after LPS and reserpine suggest that this is not the case and that both mediators are required simultaneously.

Combining the available evidence from our experimental observations with the hypothetical scheme presented above, one crucial prediction that emerges is that the ultimate cellular mechanism involves an obligatory change in protein synthesis. For this to occur there must be the transmission of an extracellular signal to the nucleus that involves or is promoted by some combination of the $\beta \mathrm{AR}$ and / or LPS or cytokine signaling pathways. A large body of evidence already exists demonstrating that $\beta$ AR signal transduction not only leads to rapid cellular responses but also that information is provided to the nucleus that results in alterations in gene expression for long term regulation. The most well described mechanism through which the $\beta$ AR signals to the nucleus involves PKA-C. The elements of this pathway are illustrated schematically in figure 48. Briefly PKA exists as an association of two catalytic subunits and two regulatory subunits. Each regulatory subunit binds to molecules of cAMP. After four cAMP molecules are bound the regulatory and catalytic subunits dissociate. The catalytic subunit can now translocate to the nucleus where it promotes the phosphorylation of CREB1. A CREB1 dimer is created by the combination of phosphorylated CREB proteins this then binds to the CREB binding protein (CPB) which activates the transcription factor cyclic AMP response element (CRE) (Boutillier et. al., 1992; Cooper, 1997). Our current research, however, suggests that while $\beta$ AR signal transduction alone is necessary, it is not sufficient to cause the hypothesized alteration in protein synthesis responsible for the increase in IHR. There apparently needs to be some synergism between LPS or cytokine signaling mechanisms and the $\beta$ AR mediated pathways. 
Identifying sites at which overlap between these signaling cascades exits should provide direction for further investigation.

Several signaling pathways have been proposed for LPS and the cytokines including the JNK(Jun N-terminal Kinase)/SAPK (Stress Activated Protein Kinase )/p38 MAP (Mitogen Activated Protein) kinase cascades and the JAK-STAT (Janus kinase- Signal Transducers and Activators of Transcription) pathway (see figure 48). In the MAP kinase (MAPK) pathways a G protein is activated, usually via an extracellular receptor, which, in turn, activates a cascade of serine-threonine kinases the last of which is capable of translocating to the nucleus where it phosphorylates a ternary complex factor (TCF) including Elk-1 and SAP1. The TCFs then form a complex with the serum response factor (SRF) to bind the serum response element (SRE). Interestingly, both the SRE and CRE are thought to require activation to transcribe the cFOS gene. JNK/SAPK (same pathway) also phosphorylate JUN to activate this protein so that it can either homodimerize or heterodimerize with FOS to form activator protein 1 (AP1) and p38 phosphorylates heat shock proteins. The JAK-STAT pathway results in a STAT homodimer that can translocate and activate transcription and JAK which can activate the G-proteins that simulate the MAPK cascades (For recent review of these topics see Cano and Mahadevan, 1995; Curran and Morgan, 1995; Cohen, 1997; Cooper 1997; Janknecht et. al., 1995; Kyriakis and Avruch, 1996; Ransohoff, 1998; Whitmarsh and Davis, 1996).

All of these pathways have been studied in the myocardium particularly in response to the cellular stress of ischemia and stress resulting in myocardial hypertrophy (Bogoyevitch et. al., 1996; Hefti et. al., 1997; Page and Doubell, 1996). Our results indicate that both $\beta$ AR stimulation and LPS or cytokine activity are required to increase IHR. It has not been 
demonstrated conclusively if either one or both messages go to the nucleus. If both signals are transmitted to the nucleus it seems likely that there may be interaction between the two pathways in order to increase IHR. These potential sites of interaction are illustrated in figure 48 .

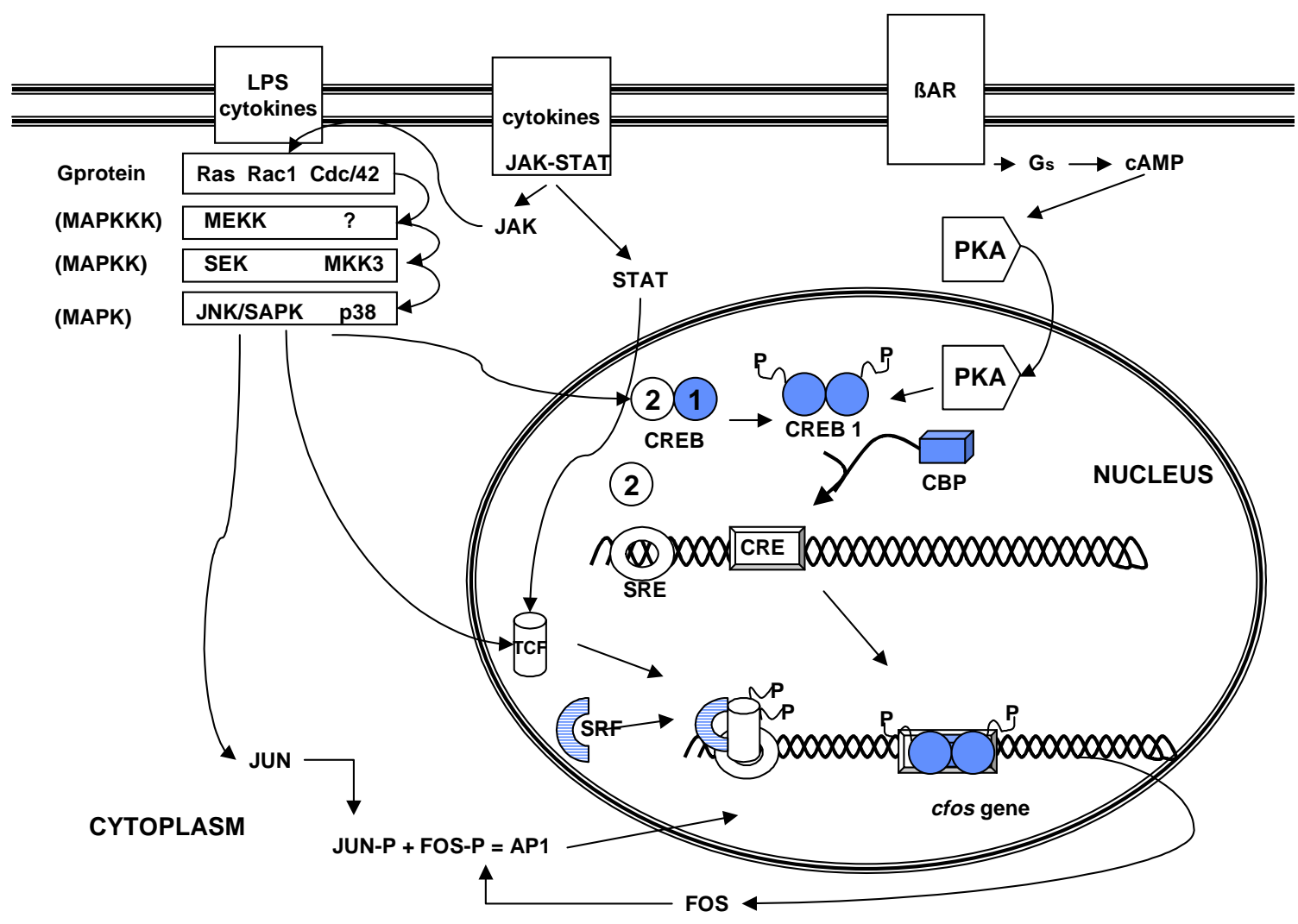

Figure 48. Figure represents some of the described mechanisms by which the $\beta A R$ and cytokines signal to the nucleus. Possible sites of interaction include at the CRE and SRE promoter sites for the cfos gene, both of which are required for transcription. Interaction at the site of release of CREB1 from CREB2 binding has been proposed as a mechanism of long term potentiation (Bailey et. al, 1996). Also there could be interaction after the phosphorylation of Jun and Fos to form the activator protein 1 (AP1) which returns to the nucleus to induce further transcriptional changes.

The last question which these experiments sought to answer was the relationship, if any, between inotropic and chronotropic changes during the development of sepsis. Our 
results demonstrated that there was a significant decrease in contractile performance at four hours but not at 16 hours following LPS administration. The converse was demonstrated with regard to an elevation in IHR, which was not elevated at four hours but was at 16 hours after endotoxin administration. The findings of contractile dysfunction at four hours confirm the findings of others using similar doses and time courses. The work presented here further demonstrates that in the rat an elevation in HR is associated with a worsening of contractile performance. What is particularly interesting is the finding of an elevation in IHR after the contractile dysfunction has returned to normal. This raises several teleological questions. Would it be beneficial for the organism to have a mechanism whereby it could increase its IHR? Is this response purely pathological or is it a normal compensatory response gone awry? These questions can probably never be fully answered nor is it the author's intent to return to the metaphysical discussion of heart rate we left behind some 300 years ago. The organism increases HR via the autonomic nervous system when it requires an immediate increase in the delivery of oxygenated blood. The increased demands in terms of oxygen and energy requirements, however, make this a costly compensation. Additionally, as was discussed earlier, in many situations the increase in HR jeopardizes contractility resulting in either no net change or a diminution of cardiac pump function. In this scenario the increase in HR, the price of which could ultimately jeopardize the heart itself, results in little gain. The detrimental effects of this elevation are particularly evident when the HR is elevated for a long period of time. Conversely, the heart, unlike other organs, is perfused exclusively in diastole. Perfusion pressure is therefore dependent on the mean pressure and the ratio of the decay time of aortic pressure in diastole and the duration of diastole. The decay time is predicted by arterial compliance. As peripheral resistance decreases the decay time 
decreases. In situations like sepsis where there is a marked reduction in the systemic vascular resistance the diastolic pressure therefore decays more quickly, and perfusion pressure rapidly decreases. One way the heart could accommodate for this reduced perfusion would be to increase HR (decrease the heart period) to maintain diastolic pressure. This has been postulated to be the reason for the differences in HR in mammals and why smaller animals tend to have much higher heart rates (Westerhof and Elzinga, 1993). In this case an intrinsic increase in heart rate would spare the organism from having to continually produce neurotransmitter to increase HR. Is an elevation of IHR a long term means of adaptation and the autonomic nervous system a short term system of accommodation? Along these lines, others have postulated that sinoatrial node may act as a servomechanism (James, 1973). By resetting the servomechanism to a higher IHR less autonomic stimulation would be required to achieve the same result.

While the benefits of decreasing the intrinsic heart rate following exercise training are clear the benefit of an increase in IHR are less so. Our experiments found that increasing the heart rate outside of the autonomic nervous system jeopardized contractility and jeopardized cardiac pump function. Direct extrapolation of rat heart rate observations to the human is fraught with more problems than the usual interspecies comparison because the existence of a positive force-frequency relationship in the rat is a matter of some debate. There are, however, pathologic states similar to sepsis where increasing heart rate in the human results in a decrease in force of contraction (Schmidt et. al., 1995; Just, 1996). Additionally, if the $\beta \mathrm{AR}$ is dysfunctional, as many have proposed, and the increase in HR involves mechanisms outside the autonomic nervous system, any increase in rate in the human would likely decrease the force of contraction. The suggestion that a long term tachycardia may be 
detrimental to the organism is increasingly recognized. In disease states in which there is a prolonged elevation of heart rate, such as hypertension, congestive heart failure and burns, the use of beta-blockers to slow rate is increasingly being advocated. Burned children treated with propranolol show a decrease in heart rate by 10 to $16 \%$ with no effect on blood pressure or tissue perfusion (Baron et. al., 1997). In patients with congestive heart failure the latest generation $\beta$-blocker, carvedilol, has been demonstrated to decrease mortality by $65 \%$, although its mechanism of action is unclear (Feurerstein et. al., 1997). Heart rate also been shown to be an important predictor of hypertension and of greater importance a major risk factor in predicting cardiovascular and noncardiovascular death (Habib, 1997; Palatini and Julius, 1997). In a prospective study of operative patients, those treated with atenolol in the perioperative period were shown to have a decreased incidence of cardiac deaths up to two years later (Mangano et. al., 1996). These studies, and our own experimental observations demonstrate that in situations of stress on the myocardium tachycardia can contribute to significant morbidity and mortality. Because the IHR appears to function as a set point that is further regulated by the autonomic nervous system, $\beta$-blockers could effectively lower HR (though not IHR) in septic patients. Whether this therapy could beneficially be extended to sepsis is unknown. Experimental evidence has suggested that sympathetic denervation, either chemically or surgically, is protective to end organs, and decreases mortality in sepsis (Bolton and Aluk, 1978; Filkins, 1979; Palmerio, 1963; Zetterstrom, 1964). We observed no decrease in mortality with reserpine pretreatment. Parker's group found in human sepsis that a lower initial HR $(<106)$, a decrease in HR in the first 24 hours by greater than 18 beats per minute, or a 24 hour HR of $<95$ are predictive of survival. This normalization of HR was coupled with a return of the stroke volume index towards normal. The authors' speculated 
that this "probably represents a normalization of the peripheral cardiovascular abnormality which led to a high HR" (Parker et. al., 1987). Our research suggests that an alternative hypothesis would be that those patients with an elevated HR might have had an ongoing pathologic elevation of IHR. Besides sepsis we know of one other disease process in which there may be an elevation of IHR. In the syndrome of "inappropriate sinus tachycardia" there is supersensitivity to $\beta$ AR agonists and a 30\% elevation of IHR (Morillo, et. al., 1994). The cause of this disease process is unknown.

In conclusion, the importance of the heart rate in human disease states has been recognized since the time of the earliest medical recordings. That the heart beat is not dependent upon nervous input and is instead myogenic in origin is no longer the subject of debate. More recently, interest in determining the intrinsic heart rate, or the rate without any nervous or humoral input, has developed. These experiments have led to the discovery that the IHR is decreased with age and exercise. This dissertation proposes the first inducible disease state, to our knowledge, in which the IHR is elevated, sepsis. In addition our work further implicates the $\beta A R$ as a necessary participant in the induction of the increase in IHR but also demonstrates that the change outlasts the ongoing stimulation of this receptor and yet a persistent elevation in IHR remains. Further work has been proposed to identify the signal to the nucleus that we hypothesize must occur to alter protein synthesis and induce this change in intrinsic heart rate. The apparent complexity of this process leads us to often agree with Harvey as he noted in 1628:

'When I first tried animal experimentation for the purpose of discovering the motions and functions of the heart by actual inspection and not by other people's books, I found it so truly difficult I almost believed with Fracastorius, that the motion of the heart was to be understood by God alone.'

William Harvey, MD 


\section{BIBLIOGRAPHY}

Abel FL. "Myocardial dysfunction in experimental shock" in Pathophysiology of shock, sepsis and organ failure Schlag G, Redl H eds. New York: Springer-Verlag, 1993

Ackerman U, Khanna J, Irizawa TG. "Atrial natriuretic factor alters autonomic interactions in the control of heart rate in conscious rats." Can J Physiol Pharmacol 66:930-936, 1988

Adams HR, Baxter CR, Parker JL, Watts NB. "Contractile function and rythmicity of cardiac preparations from Escherichia coli endotoxin-shocked guinea pigs." Circ Shock 13:241-253, 1984

Adams HR, Parker JL, Laughlin MH. "Intrinsic myocardial dysfunction during endotoxemia: dependent or independent of myocardial ischemia." Circ Shock 30:6376,1990

Adelman HB: The Embryological Treatises of Hieronymus Fabricus of Aquapendente. Ithaca, NY: Cornell University Press, 1942

Akselrod S, Gordon D, Ubel FA, et.al. "Power spectrum analysis of heart rate fluctuations: a quantitative probe of beat to beat cardiovascular control." Science 213:220-222, 1981

Anrep GV and Segall HN. "The central and reflex regulation of the heart rate." J Physiol 61:215-231, 1926

Ansanay H, Dumuis A, Sebben M, Bockaert J, Fagni L. "cAMP-dependent, long-lasting inhibition of K+ current in mammalian neurons." Proc Natl Acad Sci 92:6635-6639, 1995

Appstein CS, Mueller M, Hood WB. "Ventricular contracture and compliance changes with global ischemia and reperfusion, and their effect on coronary resistance in the rat." Circ Res 41:206-212, 1977

Archer LT, Beller BK, Drake JK, Whitsett TL, Hinshaw LB. "Reversal of myocardial dysfunction in endotoxin shock with insulin." Can J Physiol Pharmacol 45:132-138, 1978

Arden WA, Fiscus RR, Wang X, et. al. "Elevations in circulating calcitonin gene related peptide correlate with hemodynamic deterioration during endotoxic shock in pigs." Circ Shock 42:147-153, 1994

Armour JA, Yuan BX, Butler CK. "Cardiac responses elicited by peptides administered to canine intrinsic cardiac neurons.” Peptides 11:753-761, 1990 
Atchison DJ, Ackermann U. "Influence of atrial natriuretic factor on autonomic control of heart rate.” Am J Physiol 258:R718-723, 1990

Ayala A, Perrin MM, Kiscala JM, Entl W, Chaudry IH. "Polymicrobrial sepsis selectively activates peritoneal but not alveolar macrophage to release inflammatory mediators (IL-1, IL-6, and TNF).” Circ Shock 36:191-199, 1992

Baharami S, Redl H, Schlag G. "Models of endotoxemia in rodents." in Pathophysiology of shock, sepsis and organ failure Schlag G, Redl H eds. 1010-1050 New York: Springer-Verlag, 1993

Bailey CH, Bartsch D, Kandel ER. "Toward a molecular definition of long-term memory storage.” Proc Natl Acad Sci 93:13445-13452, 1996

Bainbridge FA. "The influence of venous filling upon the rate of the heart." J Physiol 50:60-84, 1915

Baker SP, Pitha J. "Irreversible blockade of beta adrenoceptors and their recovery in the rat heart and lung in vivo." J Pharmacol Exp Ther 220:247-251, 1982.

Barker LA, Winberry SL, Smith LW and McDonough KA "Supersensitivity and changes in the active population of beta adrenoceptors in right atria in early sepsis." JPET 252:675-681, 1990

Baron PW, Barrow RE, Pierre EJ, Herndon DN. "Prolonged use of propranolol safely decreases cardiac work in burned children.” J Burn Care Rehabil 18:223-227, 1997

Benedict CR and Rose JA. "Arterial norepinephrine changes in patients with septic shock." Circ Shock 38:165-172, 1992

Bensard DD, Banerjee A, McIntyre RC, Berens RL; Harken AH. "Endotoxin disrupts $\beta$ adrenergic signal transduction in the heart." Arch Surg 129:198-205, 1994

Bhagat B, Cavanagh D, Merrild BN, Rana MW, Rao PS. "Noradrenaline and tyramine action on isolated atrial muscle of endotoxin treated guinea pigs." $\mathrm{Br} \mathrm{J}$ Pharmacol 39:688-695, 1970

Bhagat B, Rao PS, Cavanaugh D. "Contractility of endotoxic atria." Adv Myocardiol 2:199207,1980

Bilder GE, Siegl Pk, Schofield TL, Friedman PA. "Chronotropic simulation : a primary effector for release of atrial natriuretic factor." Circ Res 64:799-805, 1989

Blinks JR. "Positive chronotropic effect of increasing right atrial pressure in the isolated mammalian heart." Am J Physiol 186:299-303, 1956 
Bogoyevitch MA, Gillespie-Brown J, Ketterman AJ, et. al., "Stimulation of the stressactivated mitogen-activated protein kinase subfamilies in perfused heart." Circ Res 79:162-173, 1996

Bohm M. "Alterations of $\beta$-adrenoceptor-G-protein-regulated adenylyl cyclase in heart failure." Mol Cell Biochem 147:147-160, 1995

Boillot A, Massol J, Maupoil V et. al. "Myocardial and vascular adrenergic alterations in a rat model of endotoxin shock: reversal by an anti-tumor necrosis factor-alpha monoclonal antibody." Crit Care Med 25:504-511, 1997

Boltan WK and Aluk NO. "Study of chemical sympathectomy n endotoxin-induced lethality and fibrin deposition." Kidney Int 13:263-270, 1978

Bolter CP. "Intrinsic cardiac rate regulation in the anaesthetized rabbit." Acta Physiol Scand 151:421-428, 1994

Bolter CP and Atkinson KJ. "Maximum heart rate responses to exercise and isoproterenol in the trained rat." Am J Physiol 254:R834-R839, 1988

Bolter CP and Sanders WD. "Interactive effect of acetylcholine and norepinephrine on the sinoatrial node of the trained rat." J Auton Nerv Syst 25:83-85, 1988

Bolter CP, Hughson RL, Critz JB. "Intrinsic rate and cholinergic sensitivity of isolated atria from trained and sedentary rats." Proc Soc Exp Biol Med 144:364-367, 1973

Bone RC. "Immunologic dissonance: a continuing evolution of our understanding of the systemic inflammatory response syndrome (SIRS) and the multiple organ dysfunction syndrome (MODS).” Ann Int Med 125:680-687, 1996

Bone RC, Balk RA, Cerra FB, et. al. "Definitions for sepsis and organ failure and guidelines for the use of innovative therapies in sepsis." Chest 101:1644-1645, 1992

Boundy VA, Chen J and Nestler EJ. "Regulation of cAMP-dependent protein kinase subunit expression in CATH.a and SH-SY5Y cells." J Pharmacol Exp Ther 286:1058-1065, 1998

Boutillier AL, Barthel F, Roberts JL, Loeffler JP. “ $\beta$-adrenergic stimulation of cFOS via protein kinase $\mathrm{A}$ is mediated by cAMP regulatory element binding protein (CREB)dependent and tissue-specific CREB-independent mechanisms in corticotropic cells." Jour Biol Chem 267:23520-23526, 1992

Bowditch HP. "On the peculiarities of excitability which the fibres of cardiac muscle show." Translated by Schaefer J, Deppert W, Lie RK, Lohff B, Noble MIM. In The Interval Force Relationship of the Heart: Bowditch Revisited ed Noble MIM and Seed WA. 3-42 Cambridge, UK: University Press, 1992 
Bradford MM. "A rapid and sensitive method for the quantitation of microgram quantities of protein utilizing the principle of protein-dye binding." Anal Biochem. 72:248-54, 1976

Brady AJ, Warren JB, Poole-Wilson PA, et.al. "Nitric oxide attenuates cardiac myocyte contraction.” Am J Physiol 265:H167-H172, 1993

Braunwald E.: "On the differences between the heart's output and its contractile state (editorial)." Circulation 43:171-174, 1971

Braunwald E: "Regulation of the circulation.” NEJM 290:1124-1129, 1974

Breasted JH trans. The Edwin Smith Surgical Papyrus published in facsimile and hieroglyphic transliteration with translation and commentary in two volumes by James Henry Breasted. Chicago: University of Chicago Press, 1930

Brooks C McC. The Sinoatrial Pacemaker of the Heart. Springfield, IL: C.C. Thomas, 1972

Brooks, C McC and Lange G. "Interaction of myogenic and neurogenic mechanisms that control heart rate." Proc Natl Acad Sci 74:1761-1762, 1977

Brooks C McC, Lu HH, Lange G, et. al. "Effects of localized stretch on the sinoatrial node region of the dog heart." Am J Physiol 211:1197-1202, 1966

Brusting JR, Schuil HA, Zijlstra WG. "Intrinsic heart rate in the dog determined by pharmacologic dennervation.” Am J Physiol 245:H592-H597, 1983

Bunnell E and Parrillo JE "Cardiac dysfunction during septic shock." Clin Chest Med 17:237-248, 1996

Bush EN and Vollmer RR. "Effects of dietary sodium restriction on heart rate control in rats.” Am J Physiol 244:R264-272, 1983

Campbell DL, Rasmusson RL, Strauss HC. "Ionic current mechanisms generating vertebrate primary cardiac pacemaker activity at the single cell level: an integrative view." Ann Rev Physiol 54:279-302, 1992

Campbell KL, and Forse RA. "Endotoxin-exposed atria exhibit G protein based deficits in inotropic regulation." Surgery 114:471-479, 1993

Cann M, Stevenson T, Fiallos E. "Depressed cardiac performance in sepsis." Surg Gynecol Obstet 134:759-763, 1972

Cano E and Mahadevan LC. "Parallel signal processing among mammalian MAPKs" TIBS 20:117-122, 1995 
Casteels R and Login IS. "Reserpine has a direct action as a calcium antagonist on mammalian smooth muscle cells." J Physiol 340:403-414, 1983

Cavero I, Riggenbach H, Wall M, Gerold M. "Analysis of cardiac chronotropic responses to some autonomic blocking agents in conscious trained dogs." Eur J Pharmacol 39:193-202, 1976

Chandler MP and DiCarlo SE. "Acute exercise and gender alter cardiac autonomic tonus differently in hypertensive and normotensive rats." Am J Physiol 274:R510-R516, 1998

Chaudry IH, Ayala A, Singh G, Wang P, and Hauptman JG. "Rodent models of endotoxemia and sepsis." in Pathophysiology of shock, sepsis and organ failure Schlag G, Redl H eds. 1048-1059 New York: Springer-Verlag, 1993

Cherqui C, Babire H, Fornier B, Schmitt H. "Participation of sympathetic and vagal tones in the hypotensive and bradycardic effects of some 5-HT1 like receptor agonists in the rat." Arch Int Pharmacodyn Ther 29618-28, 1988

Chiao JJC, Jones WG, Shires GT III, Barber AE, Shires GT. "Effect of sepsis on intracellular sodium activity, sodium concentration, and water content in the thermal injured rat." Circ Shock 38:42-49, 1992

Cho KW, Seul KH, Kim SH, Seul KM, Koh GY. "Atrial pressure, distension, and pacing frequency in ANP secretion in isolated perfused rabbit atria." Am J Physiol 260:R3946, 1991.

Chung MK, Gulick TS, Rotondo RE. "Mechanism of cytokine inhibition of $\beta$-adrenergic agonist stimulation of cyclic AMP in rat cardiac myocytes.” Circ Res 67:753-763, 1990

Coalson JJ, Woodruff K, Greenfield LJ, Guenter CA and Hinshaw LB. "Effects of digoxin on myocardial ultrastructure in endotoxin shock." Surg Gynecol Obstet 135:908-912, 1972

Cohen DM. "Mitogen-activated protein kinase cascades and the signalling of hyperosmotic stress to immediate early genes." Comp Biochem Physiol 117A:291-299, 1997

Cooper GM. The Cell: A Molecular Approach. Washington, DC: ASM Press, 1997

Corr L. "Neuropeptides and the conduction system of the heart." Int J Cardiol 35:1-12, 1992

Craft N and Schwartz JB. "Effects of age on intrinsic heart rate, heart rate variability, and AV conduction in healthy humans.” Am J Physiol 268:H1441-H1452, 1995 
Cunnion RE and Parrillo JE. "Myocardial dysfunction in sepsis." Critical Care Clinics 5:99118,1989

Cunnion RE, Schaer GL, Parker MM, Natanson C, Parrillo JE. "The coronary circulation in human septic shock." Circulation 73:637-644, 1986

Curran T and Morgan JI. "Fos: an immediate-early transcription factor in neurons." Jour Neurobiol 26:403-412, 1995

Dabire H, Chaouche-Teyara K, Cherqui C, Fournier B, Schmitt H. "Pharmacologic analysis of cardiac effects of 5-HT and some 5-HT receptor agonists in the pithed rat." Fundam Clin Pharmacol 6:237-245, 1992

Deaciuc IV and Spitzer JA. "Calcium content in liver and heart and its intracellular distribution in liver during endotoxicosis and sepsis in rats." Cell Calcium 8:365-376, 1987

DeLuca A, Rand MJ. "Involvement of alpha 1-adrenoceptors in chronotropic responses to exogenously released amines in the pithed rat." Clin Exp Pharmacol Physiol 15:3341,1988

Dhainaut JF, Huyghebaert MF, Monsallier JF, et. al. "Coronary hemodynamics and myocardial metabolism of lactate, free fatty acids, glucose, and ketones in patients with septic shock." Circulation 75:533-541, 1987

Dickhout JG and Lee RMKW. "Blood pressure and heart rate development in young spontaneously hypertensive rats." Am J Physiol 274:H794-H800, 1998

Doby T. Discoverers of Blood Circulation from Aristotle to the Times of Da Vinci and Harvey. New York: Abelard-Schuman, 1963

Doggrell SA. "The membrane stabilizing and $\beta 1$-adrenoceptor blocking activity of $(+)$ and $(-$ ) propranolol on the rat left atria." Gen Pharmacol 21:677-680, 1990

Doggrell SA. "Effects of bromoacetylalprenololmenthane (BAAM), an irreversible betaadrenoceptor antagonist, on the rat isolated left atria and portal vein." J Auton Pharmacol 10:333-344, 1990b.

Doring HJ. "The isolated perfused heart according to Langendorff." Phys Bohem 39:481504,1990

Dunn DL. "Gram-negative bacterial sepsis and sepsis syndrome." Surg Clin North Am 74:621-635, 1994 
Elkins RC, McCurdy JR, Brown PP and Greenfield LJ. "Effects of coronary perfusion on myocardial performance during endotoxin shock." Surg Gynecol Obstet 137:991996, 1973

Eyster JAE and Meek WJ. "The origin and conduction of the heart beat." Physiol Rev 1:143,1921

Favaretto AL, Ballejo GO, Albuquerque-Araujo WI. "Oxytocin releases atrial natriuretic peptide from rat atria in vitro that exerts negative inotropic and chronotropic action." Peptides 18:1377-1381, 1997

Feldman MD, Gwatheny JK, Phillips P, Schoen F and Morgan JP. "Reversal of the forcefrequency relationship in human heart failure." J Appl Cardiol 3:273-283, 1988

Feurerstein GZ, Bril A, Ruffolo RR. "Protective effects of carvedilol in the myocardium." Am J Cardiol 80:41L-45L, 1997

Filkins JP. "Adrenergic blockade and glucoregulation in endotoxin shock." Circ Shock 6:99-107, 1979

Finkel MS, Oddis CV, Jacbob TD, et. al. "Negative inotropic effects of cytokines on the heart mediated by nitric oxide." Science 17:387-389, 1992

Fish RE, Burns AH, Lang CH and Spitzer JA. "Myocardial dysfunction in a nonlethal, nonshock model of chronic endotoxemia." Circ Shock 16:241-252, 1985

Flavahan NA, McGrath JC. "Alpha 1-adrenoceptors can mediate chronotropic responses in the rat heart.” Br J Pharmacol 73:586-588, 1981

Fleming WW. "A review of postjunctional supersensitivity in cardiac muscle." In Neuronal and Extraneuronal Events in Autonomic Pharmacology, ed Fleming WW. 205-220 New York: Raven Press, 1984

Fleming WW. Sensitivity. Lectures in Advanced Neuropharmacology, Department of Pharmacology and Toxicology, West Virginia University. November 14 and 16, 1997

Fleming WW and Taylor DA. "Cellular mechanisms of opioid tolerance and dependence." In Pharmacology of Opioid Peptides 463-501 Amsterdam: Harwood Academic Publishers, 1995

Fleming WW and Taylor DA. "Cyclic AMP and adaptive supersensitivity in guinea pig atria.” J Neural Tansm 34S:179-185, 1991 
Fleming WW and Westfall DP. "Adaptive Supersensitivity." In Handbook of Experimental Pharmacology. Trendelenburg U and Weiner N eds. 509-559 Berlin: SpringerVerlag, 1988

Fleming WW, Westfall DP, De la Lande IS, Jellett, LB. "Log-normal distribution of equiefective doses of norepinephrine and acetylcholine in several tissues." J Pharmacol Exp Ther 181:339-345, 1972

Fye WB. "The origin of the heart beat: a tale of frogs, jellyfish, and turtles." Circulation 76:493-500, 1987

Gardey C, Thiroux G, Carli A. "Lack of alteration in cardiac $\beta$-adrenoceptor site concentration in rats treated with an adenylate cyclase-stimulating dose of endotoxin." Shock 1:359-361, 1994

Gardey C, Thiroux G, Christeff N, Carli A. "Unexpected hyperactivity of myocardial adenylate cyclase system in endotoxic male rats." Circ Shock 31:297-307, 1990

Garrard CS, Kontoyannis DA, Piepoli M. "Spectral analysis of heart rate variability in the sepsis syndrome." Clin Autonom Res 3:5-13, 1993

Garrel G, Delahaye R, Hemmings GA and Counis R. "Modulation of regulatory and catalytic subunit levles of cAMP-dependent protein kinase A in anterior pituitary cells in response to direct activation of protein kinases A and C or after $\mathrm{GnRH}$ stimlation." Neuroendocrinology 62:514-522, 1995

Gaskell WH. "On the innervation of the heart, with special reference to the heart of the tortoise." J Physiol 4:43-127, 1881

Gauer OH, Henry JP, Sieker HO. "Cardiac receptors and fluid volume control." Progr Cardiovasc Dis 4:1-26, 1961.

Geison G. "The Royal Institution Lectures of 1869." In Michael Foster and the Cambridge School of Physiology, Princeton: Princeton University Press, 1978

Gilbert RP. "Mechanisms of the hemodynamic effects of endotoxin." Physiol Rev 40:245279,1960

Giuliani S, Tramontana M, Lecci A, Maggi CA. "Effect of nociceptin on heart rate and blood pressure in anaesthetized rats." Eur J Pharmacol 333 :177-179, 1997

Godin PJ, Fleisher LA, Eidsath A, et.al. "Experimental human endotoxemia increases cardiac regularity: results from a prospective, randomized crossover trial." Crit Care Med 24:1117-1124, 1996 
Goldfarb RD. "Evaluation of ventricular performance in shock." Circ Shock 15:281-301, 1985

Goldstein B, Kempski MH, Stair D, et. al. "Autonomic modulation of heart rate variability during endotoxin shock in rabbits." Crit Care Med 23:1694-1702, 1995

Goodyear AVN. "Left ventricular function and tissue hypoxia in irreversible hemmorhagic and endotoxin shock." Am J Physiol 212:444-450, 1967

Gorman AJ and Proppe DW. "Mechanisms producing tachycardia in conscious baboons during environmental heat stress.” J Apply Physiol 56:441-446, 1984

Gotloib L, Shostak A, Galdi P, Jaichenko J, Fudin R. "Loss of microvascular negative charges accompanied by interstitial edema in septic rats' heart." Circ Shock 36:45-56, 1992

Gotoh H. "Phosphorylation and adrenergic chronotropism and inotropism in guinea pig cardiac muscles." Life Sciences 56:1655-1663, 1995

Guest TM, Ramanathan AV, Tuteur PG, et. al. "Myocardial injury in critically ill patients: a frequently unrecognized complication." JAMA 273:1945-1949, 1995

Haase H, Bartel S, Karzewski P, Morano I, Krause EG. "In-vivo phosphorylation of the cardiac L-type calcium channel beta-subunit in response to catecholamines." Mol Cell Biochem 163-164:99-106, 1996

Habib G. "Reappraisal of the importance of heart rate as a risk factor for cardiovascular morbidity and mortality.' Clin Therap 19:Suppl A39-52, 1997

Halinen MO, Hakumaki MOK, Sarajas HSS. "Circulatory reflex responses during the initial stage of feline endotoxin shock.” Acta Physiol Scand 101:264-269, 1977

Haller A. Dr. Albert Haller's Physiology. London: G. Robinson, 1777

Harden TK. "Agonist-induced desensitization of the $\beta$-adrenergic receptor-linked adenylate cyclase.” Pharmacol Rev 35:5-32, 1983

Harri M and Kuusela P. "Effects of the adrenergic nervous system on training-induced cardiac enlargement, and on the intrinsic rate and phenylephrine sensitivity of isolated atria.” Can J Physiol Pharmacol 60:1125-1130, 1982

Harvey W. "An anatomical dissertation upon the movement of the heart and blood in animals." Translated Leake CD, Springfield IL: Charles C. Thomas, 1930

Hefti MA, Harder BA, Eppenberger HM, Schaub MC. "Signaling pathways in cardiac myocyte hypertrophy." J Mol Cell Cardiol 29:2873-2892, 1997 
Heiffer MN, Murray RL, Mehlman B. "Effect of lethal doses of bacterial endotoxin (E. coli) on sympathetic neurohormones in the rabbit." Am J Physiol 198:1307-1311, 1960

Hemmings BA. "cAMP mediated proteolysis of the catalytic subunit of cAMP-dependent protein kinase." FEBS Lett 196:126-130, 1986.

Herbertson MJ, Werner HA, Wally KR. "Nitric oxide synthase inhibition partially prevents decreased LV contractility during endotoxemia.” Am J Physiol 270:H1979-H1984, 1996

Heslop KE and Curzon G. "Depletion and repletion of cortical tissue and dialysate 5-HT after reserpine." Neuropharmacology 33:567-573, 1994

Hinshaw LB. "Myocardial function in endotoxin shock." Circ Shock 1[suppl]:43-45, 1979

Hinshaw LB, Brale CM, Emerson TE, Jordan MM, Masucci FD. "Participation of the sympathoadrenal system in endotoxin shock.” Am J Physiol 207:925-930, 1964

Hinshaw LB, Greenfield LJ, Owen SE, Archer LT, Guenter CA. "Cardiac response to circulating factors in endotoxin shock." Am J Physiol 222:1047-1053, 1972

Hinshaw LB, Greenfield LJ, Owen SE, Black MR, Guenter CA. "Precipitation and reversal of myocardial failure in endotoxin shock." Surg Gynecol Obstet 136:39-48, 1972

Hirata Y, Mitaka C, Sato K, et. al. "Increased circulating adrenomedullin, a novel vasodilatory peptide, in sepsis." J Clin Endocrinol Metab 81:1449-1453, 1996

Hiwatari M, Satoh K, Angus JA, Johnston CI. "No effect of atrial natriuretic factor on cardiac rate, force and transmitter release." Clin Exp Pharmacol Physiol 13:163-168, 1986.

Hock CE, Yin K, Yue G, Wong PYK. "Effects of inhibition of nitric oxide synthase by aminoguanidine in acute endotoxemia." Am J Physiol 272:H843-H850, 1997

Hoff HE. "Vagal stimulation before the Webers." Ann Med Hist N S 8:138-144, 1936

Hoffman BF and Kelly JJ. "Effects of rate and rhythm on contraction of the rat papillary muscle." Am J Physiol 197:1199-1204, 1959

Horowitz $\mathrm{M}$ and Meiri U. "Central and peripheral contributions to control of heart rate during heat acclimation." Plugers Arch 422:386-392, 1993

Hotchkiss RS and Karl IE. "Calcium: a regulator of the inflammatory response in endotoxemia and sepsis." New Horiz 4:58-71, 1996 
Houque AM, Marczin N, Catravas JD, Fuchs LC. "Anesthesia with sodium pentabarbital enhances lipopolysaccharide-induced cardiovascular dysfunction in rats." Shock 6:365-370, 1996

Hsu C, Yang SL, Hsu SP, Hsu HK, Liu MS. "Differential activation of protein kinase A in various regions of myocardium during sepsis.” J Surg Res 71:161-165, 1997

Huang XY, Morielli AD, Peralta EG. "Molecular basis of cardiac potassium channel stimulation by protein kinase A." Proc Natl Acad Sci 91:624-628, 1994

Hughson RL, Sutton JR, Fitzgerald JD and Jones NL. "Reduction of intrinsic sinoatrial frequency and norepinephrine response of the exercised rat." Can J Physiol Pharmacol 55:813-820, 1977

Huttemeier PC, Ritter EF, Benveniste H. "Calcitonin gene-related peptide mediates hypotension and tachycardia in endotoxic rats.” Am J Physiol 265:H767-H769, 1993

Iwayama T, Fleming WW and Burnstock G. "Ultrastructure of mitochondria in atrial muscle associated with depression and suspersensitvity produced by reserpine." Jour Pharmacol Exp Ther 184:95-105, 1973

Jacobs DO, Kobayashi T, Imagire J, et. al. "Sepsis alters skeletal muscle energetics and membrane function. Surgery 110:318-326, 1991

James TN. “The sinus node as a servomechanism.” Circ Res 32:307-313, 1973

Jandhyala BS, Cavero I, Adams HR et. al. "Cardiovascular effects of chronic reserpine administration in mongrel dogs." Eur J Pharmacol 16:261-270, 1971

Janknecht R, Cahill MA, Nordheim A. "Signal integration at the c-fos promoter." Carcinogenesis 16:443-450, 1995

Japundzic N, Grichois ML, Zitoun P, Laude D and Elghozi JL. "Spectral anlaysis of blood pressure and heart rate in conscious rats: effects of autonomic blockers." Jour Autonom Nerv Syst 30:91-100, 1990

Jensen D. Intrinsic cardiac rate regulation. New York: Appleton-Century-Crofts, 1971

Johnson MD and Kotchen TA. "Role of epinephrine in the development of hypertension in Dahl salt-sensitive rats." Hypertension 16:282-289, 1990

Johnson ML and Billiar TR. "Roles of nitric oxide in surgical infection and sepsis." World J Surg 22:187-196, 1998

Johnson SM and Fleming WW. "Mechansims of cellular adaptive sensitivity changes: applications to opioid tolerance and dependence." Pharmacol Rev 41:435-488, 1989 
Jones SB and Romano FD "Plasma catecholamines in the conscious rat during endotoxicosis.” Circ Shock 14:189-201, 1984

Jones SB and Romano FD "Dose and time dependent changes in plasma catecholamines in response to endotoxin in conscious rats." Circ Shock 28:59-68, 1989

Jones SB and Romano FD "Myocardial beta adrenergic receptor coupling to adenylate cyclase during developing septic shock." Circ Shock 30:51-61, 1990.

Jones SB, Kotsonis P, Majewski $\mathrm{H}$ "Edotoxin enances norepinephrine release in the rat by peripheral mechanisms." Shock 2:370-375, 1994

Jose AD. "Effect of combined sympathetic and parasympathetic blockade on heart rate and cardiac function in man." Am J Cardiol 18:476-478, 1966

Jose AD and Collison D. "The normal range and determinants of the intrinsic heart rate in man." Cardiovasc Res 4:160-167, 1970

Jose AD and Stitt F. "Cardiac function after combined beta adrenergic and cholinergic blockade. Relationship of intrinsic rate to contractile force of the heart in dogs." Circ Res 20-21:suppl 111:231-242, 1967

Jose AD and Stitt F. "Effects of hypoxia and metabolic inhibitors on the intrinsic heart rate and myocardial contractility in dogs." Circ Res 25:53-66, 1969

Jose AD, Stitt F and Collison D. "The effects of exercise and changes in body temperature on the intrinsic heart rate in man." Am Heart J 79:488-498, 1970

Jose AD and Taylor RR. "Autonomic blockade by propranolol and atropine to study intrinsic myocardial function in man." J Clin Invest 48:2019-2031, 1969

Just H. "Pathophysiological targets for beta-blocker therapy in congestive heart failure." Eur Heart J 17 Suppl B:2-7, 1996

Kappagoda CT, Linden RJ, Snow HM. "The effect of stretching the superior vena cavalright atrial junction or the right atrial receptors in the dog." J Physiol 227:875-887, 1972

Karlstad MD, Sayeed MM. "Effect of endotoxic shock on skeletal muscle intracellular electrolytes and amino acid transport." Am J Physiol 252:R674-R680, 1987

Katz AM. Physiology of the Heart $2^{\text {nd }}$ edition. New York, New York: Raven Press, 1992

Keith A and Flack M. "The form and nature of the muscular connections between the primary divisions of the vertebrate heart.” J Anat Physiol 41:172-189, 1906-1907 
Keller RS, Jones JJ, Kim KF, et. al. "Endotoxin-induced myocardial dysfunction: is there a role for nitric oxide." Shock 4:338-344, 1995

Klabunde RE and Cotson AF. "Nitric oxide synthase inhibition does not prevent cardiac depression in endotoxic shock." Shock 3:73-78, 1995

Kleinman WM, Krause SM, Hess ML. "Differential subendocardial perfusion and injury during the course of gram-negative endotoxemia." Adv Shock Res 4:139-152, 1980

Koch-Weser J and Blinks JR. "The influence of the interval between beats on myocardial contractility." Pharmacol Rev 15:601-652, 1963

Knowlton FP and Starling HP. "The influence of variations in temperature and blood pressure on the performance of the isolated mammalian heart." J Physiol 44:206-219, 1912

Kramer CY. "Extension of multiple range tests to group means with unequal numbers of replications." Biometrics 12:307-310, 1956

Kreider MS, Golberg PB and Roberts J. "Effect of age on neuronal uptake in rat heart." J Pharmacol Exp Ther 231:367-372, 1984

Krosl P and Pretorius J. "Myocardial dysfunction in experimental septic shock." in Pathophysiology of shock, sepsis and organ failure Schlag G, Redl H eds. 835-852 New York: Springer-Verlag, 1993

Krosl P, Pretorius J, Redl H, Schlag G. "Myocardial function in septic sheep.” Shock 1:325334, 1994

Kumar A and Parrillo JE. "Clinical manifestations of cardiovascular dysfunction in sepsis." in Pathophysiology of shock, sepsis and organ failure Schlag G, Redl H eds. 859-881 New York: Springer-Verlag, 1993

Kumar A, Brar R, Wang P, et. al. "Role of nitric oxide and cGMP in human septic seruminduced depression of cardiac myocyte contractility." Am J Physiol 276:R265-276, 1999

Kunkel SL, Chensue SW, Standiford TJ, Strieter RM. "Endotoxin-dependent cytokine networks." In Endotoxin and the Lungs ed. Brigham KL. 305-320 New York: Marcel-Decker, 1994

Kutsky P and Parker JL. "Calcium fluxes in cardiac sarcolemma and sacroplasmic reticulum isolated from endotoxin-shocked guinea pigs." Circ Shock 30:349-364, 1990 
Kuwahara M, Yayou KI, Ishii K, et.al. "Power spectral analysis of heart rate variability as a new method of assessing autonomic activity in the rat." 27:333-337, 1994-

Kyriakis JM and Avruch J. "Sounding the alarm: protein kinase cascades activated by stress and inflammation." Jour Biol Chem 271:24313-24316, 1996

Lang RE, Tholken H, Ganten D. "Atrial natriuretic factor - a circulating hormone stimulated by volume loading." Nature 314:264-266, 1985.

Leake CD. "The historical development of cardiovascular physiology." In Handbook of Physiology, Section 2: Circulation, vol I., ed. WF Hamilton, P Dow, 11-22.

Washington, D.C.: American Physiologic Society, 1962

Ledsome JR, Wilson N, Courneya CA, Rankin AJ. "Release of atrial natriuretic peptide by atrial distension.” Can J Physiol Pharmacol 63:739-742, 1985

Lefer AM. "Mechanisms of cardiodepression in endotoxin shock." Circ Shock Suppl 1:1-8, 1979

Levick JR. An Introduction to Cardiovascular Physiology. Oxford: Butterworth-Heineman, 1995

Levitski A. "From epinephrine to cyclic AMP." Science 241:800-807, 1988

Levy MN. "Neural control of the heart." In Handbook of Physiology, Section 2: The Cardiovascular System, vol. I The Heart, ed. RM Berne, N Sperelakis, SR Geiger, 581-620. Bethesda: American Physiologic Society, 1979

Levy MN and Blattberg B. "Changes in heart rate induced by bacterial endotoxin." Am J Physiol 213:1485-1492, 1967

Lewis SF, Nylander E, Gad P, Areskog NH. "Non-autonomic component in bradycardia of endurance in trained men at rest and during exercise." Acta Physiol Scand 109:297305,1980

Leyden AF, Pomerantz E, Bouchard EF. "Pharmaceutical aspects of reserpine." J Am Pharm Assoc 45:771-775, 1956

Liang P and Pardee AB. Differential Display Methods and Protocols Totowa, NJ: Humana Press, 1997

Lin HC, Thurman JC, Tranquilli WJ, Benson GJ, Olson WA. "Hemodynamic response of calves to tiletamine-zolazepam-xylazine anesthesia.” Am J Vet Res 52:1606-1610, 1991 
Lin YC, Horvath SM. "Autonomic nervous control of cardiac frequency in the exercise trained rat." J Appl Physiol 33:796-801, 1972

Liu MS. "Mechanisms of myocardial membrane alterations in endotoxin shock: roles of phospholipase and phosphorylase." Circ Shock 30:43-49, 1990

Lubbe AS, Harris PD, Garrison RN. "Systemic hemodynamic and microvascular responses in spontaneously hypertensive rats during Escherichia coli bacteremia." Circ Shock 40:157-167, 1993.

Machado BH, Krieger EM, and Salgado HC. "Changes in the intrinsic heart rate during the onset of renal hypertension." J Hypertension 5:755-759, 1987

Machado BH and Brody MJ. "Contribution of neurogenic mechanisms to control of intrinsic heart rate.” Am J Phys 256:R231-R235, 1989

Malik M. "Heart rate variability: standards of measurement, physiological interpretation, and clinical use" from the Task Force of the European Society of Cardiology and the North American society of Pacing and Electrophysiology." Eur Heart J 17:354-381, 1996

Malliani A, Pagani M, Lombardi F, Cerutti S. "Cardiovascular neural regulation explored in frequency domain.” Circulation 84:482-492, 1991

Mangano DT, Layug EL, Wallace A, Tateo I. "Effect of atenalol on mortality and cardiovaxcular morbidity after noncardiac surgery." N Engl J Med 335:1713-1720, 1996.

Markwalder J and Starling EH. "On the constancy of the systolic output under varying conditions." J Physiol 48:348-356, 1914

Marsh NA and Shattock MJ. "In celebration of 100 years of the isolated perfused heart Lagendorff revisited." J Physiol 505:69P, 1997

Matthews JM, Falakh PHJ, Lenaar P, Summers RJ. "Chronic (-) isoprenaline infusion down regulates $\beta 1$ and $\beta 2$ adrenoceptors but does not transregulate muscarinic cholinoceptors in rat heart." Naunyn Schmiedeberg's Arch Pharmacol 353:213-225, 1996

Mayow J. "Tractus quinque. Oxford 1674. (Trans., Medico-Physical Works of Mayow. Edinburgh., Alembic Club, 1907, xxiii, 331, pp. 4 ). Quoted in Hoff HE, "Vagal stimulation before the Webers." Ann Med Hist N S 8:138-144, 1936

McDonough KH. "Calcium uptake by sarcoplasmic reticulum isolated from hearts of septic rats.” Cic Shock 25:291-297, 1988 
McDonough KH, Lang CH, Spitzer JJ. "Depressed function of isolated hearts from hyperdynamic septic rats.” Circ Shock 12:241-251, 1984

McKechnie K, Dean HG, Furman BL and Paratt JR. "Plasma catecholamines during endotoxin infusion in conscious unrestrained rats: effects of adrenal demedullation and/or guanethidine treatment." Circ Shock 17:85-94, 1985

Meng X, Ao L, Brown JM, et. al. "Nitric oxide synthase is not involved in cardiac contractile dysfunction in a rat model of endotoxemia without shock." Shock 7:111118,1997

Metzler CH, Lee ME, Thrasher TN, Ramsay DJ. "Increased right or left atrial pressure stimulates release of atrial natriuretic peptides in conscious dogs." Endocrinology 119:2396-2398, 1986

Morillo CA, Klein GJ, Thakur RK, et. al. "Mechanism of 'inappropriate' sinus tachycardia." Circulation 90:873-877, 1994

Mulieri LA, Hasenfuss G, Leavitt B, Allen PD, Alpert NR. "Altered myocardial forcefrequency relation in human heart failure." Circulation 85:1743-1750, 1992

Muller-Hocker J and Haerty. "Pathomorphological aspects of the heart in septic patients." In Pathophysiology of Shock, Sepsis and Organ Failure. Schlag G and Redl H eds. 853-858 Berlin: Springer-Verlag, 1993

Muller-Werdan U, Schumann H, Fuchs R, et. al. "Tumor necrosis factor alpha is a cardiodepressant in pathophysiologically relevant concentrations without inducing inducible nitric oxide synthase or triggering serious cytotoxicity." J Mol Cell Cardiol 29:2915-2923, 1997

Muniz AE and McDonough KH. "Function of isolated hearts from septic, saline-infused, and septic, alcohol-infused rats." Alcohol Clin Exp Res 15:1067-1071, 1991

Muntzel MS, Abe A, Petersen JS. "Effects of adrenergic, cholinergic and ganglionic blockade on acute depressor responses to metformin in spontaneously hypertensive rats.” J Pharmacol Exp Therap 281:618-623, 1997

Nagata M, Franco-Cereceda A, Saria A, Amann R and Lundberg JM. "Reserpine induced depletion of neuropeptide $Y$ in the guinea-pig: tissue specific effects and mechanisms of action." J Auton Nerv Syst 20:257-263, 1987

Nakashima A, Angus JA, and Johnston CI. "Chronotropic effects of angiotensin I, angiotensin II, bradykinin and vasopressin in guinea pig atria.” Eur J Pharmacol 81:479-485, 1982 
Narasway SA, Rackow EC, Astiz ME, Karr G and Weil H. "Inotropic response to digoxin and dopamine in patients with severe sepsis, cardiac failure, and systemic hypotension." Chest 95:612-615, 1989

Natanson C, Hoffman WD, Parillo JE "Septic shock and multiple organ failure." in Critical care medicine principles of diagnosis and management Parrillo JE and Bone RC, eds. St. Louis MO: Mosby, 1995

Natanson C, Fink MP, Ballantyne HK, et. al. "Gram-negative bacteremia produces both severe systolic and diastolic cardiac dysfunction in a canine model that simulates septic shock.” Jour Clin Invest 78:259-279, 1986

Neely JR, Liebermeister H, Battersby EJ and Morgan HE. "Effect of pressure development on oxygen consumption by isolated rat heart." Am J Phys 212: 804-814, 1967

Nishio K, Akai Y, Murao Y, et. al. "Increased plasma concentrations of adrenomedullin correlate with relaxation of vascular tone in patients with septic shock." Crit Care Med 25:953-957, 1997

Noble MIM and Seed WA eds. The Interval Force Relationship of the Heart: Bowditch Revisited Cambridge, UK: University Press, 1992

Nykiel F and Glaviano W. "Adrenal catecholamines in E. coli endotoxin shock." J Appl Pysiol 16:348-350, 1961

Nylander E and Dahlstrom U. "Influence of long-term beta receptor stimulation with prenalterol on intrinsic heart rate in rats." Eur J Appl Physiol 53:48-52, 1984

Nylander E, Sigvardsson K and Kilbom A. "Training-induced bradycardia and intrinsic heart rate in rats.” Eur J Appl Physiol 48:189-199, 1982

Ori Z, Monir G, Weiss J, Saynouni X, Singer DH. "Heart rate variability." Cardiol Clin 10:499, 1992

Palatini P and Julius S. "Heart rate and cardiovascular risk.” J Hypertension 15:3-17, 1997

Palmerio C, Ming SC, Frank ED and Fine J. "Cardiac tissue response to endotoxin." Proc Soc Exp Biol Med 109:773-776, 1962

Palmerio C, Zetterstrom B, Shammash J, Euchbaum E, Frank E, Fine J. "Denervation of the abdominal viscera for treatment of traumatic shock.” N Engl J Med 269:707-716, 1963

Palsson J, Ricksten SE, Delle M, Lundin S. "Changes in renal sympathetic nerve activity during experimental septic and endotoxin shock in conscious rats." Circ Shock 24:133-141, 1988 
Pardini BJ, Jones SB and Filkins JP. "Contribution of depressed reuptake to the depletion of norepinephrine from rat heart and spleen during endotoxin shock." Circ Shock 9:129-143, 1982

Pardini BJ, Jones SB and Filkins JP. "Cardiac and splenic norepinephrine turnovers in endotoxic rats.” Am J Phys 245:H276-H283, 1983

Page C and Doubell AF. "Mitogen-activated protein kinase (MAPK) in cardiac tissues." Mol Cell Biochem 157:49-57, 1996

Parker JL and Adams HR. "Myocardial effects of endotoxin shock: characterization of an isolated heart muscle model." Adv Shock Res 2:163-175, 1979

Parker JL and Adams HR. "Contractile dysfunction of atrial myocardium from endotoxin shocked guinea pigs.” Am J Physiol 240:H954-H962, 1981

Parker MM, Shelhammer JH, Bacharach SL, et. al. "Profound but reversible myocardial depression in patients with septic shock." Ann Intern Med 100:483-490, 1984

Parker MM, Shelhamer JH, Natanson C, Alling DW, Parrillo JE. "Serial cardiovascular variables in survivors and nonsurvivors of human septic shock: heart rate as an early predictor of prognosis." Crit Care Med 15:923-939, 1987

Parnas I, Reinhold R, Fine J. "Synaptic transmission in the crayfish: increased release of neurotransmitter substance by bacterial endotoxin.” Science 171:1153-1155, 1971

Parratt JR. "Myocardial and circulatory effects of E. coli endotoxin; modification of responses to catecholamines." Br J Pharmacol 47:12-25, 1973

Parrillo JE "Septic shock in humans. Advances in the understanding of pathogenesis, cardiovascular dysfunction and therapy." Ann Int Med 113, 227-242, 1990

Parrillo JE, Burch C, Shelhamer JH, et. al. "A circulating myocardial depressant substance in humans with septic shock: septic shock patients with a reduced ejection fraction have a circulating factor that depresses in vitro myocardial cell performance." J Clin Invest 76:1539-1553, 1985

Pathak CL. "The fallacy of the Bainbridge reflex.” Am Heart J 72:577-581, 1966

Pathak CL. "Effects of changes in intraluminal pressure on inotropic and chronotropic responses of isolated mammalian hearts." Am J Physiol 194:197-199, 1958

Patten BM. "Initiation of early changes in the character of the heart beat in vertebrate embryos. Physiol. Rev. 29:31-47, 1949 
Payen D, Bernard C, Beloucif S. "Nitric oxide in sepsis." Clin Chest Med 17:333-350, 1996

Portaluppi F, Pradella A, Montanari et al. "Atrial strain is the main determinant of release of atrial natriuretic peptide." Int J Cardiol 29:297-303, 1990

Presier JC, Moulart D, Vincent JL. "Addition of alinidine, a specific bradycardic agent, to dobutamine in a canine model of endotoxic shock." Crit Care Med 20:1146-1151, 1992

Quartin AA, Schein RMH, Kett DH, Peduzzi PN. "Magnitude and duration of the effect of sepsis on survival." JAMA 277:1058-1063, 1997

Qi M, Zheng ZZ, Wurster RD and Jones SB "Mechanisms involved in the rapid dissipation of plasma epinephrine response to bacterial endotoxin in conscious rats." Am J Phys 261:R1431-R1437, 1991

Randall WC, ed. Nervous Control of Cardiovascular Function. Oxford: Oxford University Press, 1984

Ransohoff RM. "Cellular responses to interferons and other cytokines: the JAK-STAT paradigm.” NEJM 338:616-618, 1998

Raymond RM. "When does the heart fail during shock?" Circ Shock 30:27-41, 1990

Redl H, Schlag G, Baharami S, et. al. "Experimental and clinical evidence of leukocyte activation in trauma and sepsis." In Bacterial Endotoxins: Basic Science to AntiSepsis Strategies 221-245 New York: Wiley-Liss, Inc., 1994

Reitschel ET and Wagner H eds. Current Topics in Microbiology and Immnology: Pathology of Septic Shock vol 216 Berlin: Springer-Verlag, 1996

Reitschel ET, Brade H, Holst O, et. al., "Bacterial endotoxin: chemical constitution, biological recognition, host response and immunological detoxification." Curr Microbiol Immunol 216:39-81, 1996

Rice PJ, Taylor DA, Valinski WA, Head RJ and Fleming WW. "Norepinephrine depletion and sensitivity changes in rat heart induced by pretreatment with reserpine." JPET 240:764-771, 1987

Riddervold, F, Smiseth OA, Bjornerheim R, Hall C, Risoe C. "The relationship between atrial pressure, atrial dimensions and atrial natriuretic factor during pacing tachycardia in dogs." Acta Anaesthesiol Scand 35:731-735, 1991

Rigby SL, Hofmann PA, Zhong J, Adams HR and Rubin LJ. "Endotoxemia-induced myocardial dysfunction is not associated with changes in myofilament $\mathrm{Ca} 2+$ responsiveness.” Am J Physiol 274:H580-H590, 1998 
Roberts MI, Biser PS, Stadel JM, Taylor DA and Fleming WW. "Adenylyl cyclase and guanine nucleotide binding proteins in supersensitive guinea pig ventricles." Mol Pharmacol 42:784-791, 1992

Robertson FM, Offner PJ, Ciceri DP, et. al. "Detrimental hemodynamic effects of nitric oxide synthase inhibition in septic shock." Arch Surg 129:149-156, 1994

Romano FD and Jones SB. "Beta-adrenergic stimulation of myocardial cyclic AMP in endotoxic rats." Circ Shock 17:243-252, 1985

Romano FD and Jones SB. "Characteristics of myocardial $\beta$-adrenergic receptors during endotoxicosis in the rat." Am J Phys 251:R359-R364, 1986a

Romano FD and Jones SB. "Alterations in $\beta$-adrenergic stimulation of myocardial adenylate cyclase in endotoxic rats." Am J Phys 250:R358-R364, 1986b

Romanosky AJ, Giaimo ME, Shepherd RE, and Burns AH. "The effect of in vivo endotoxin on myocardial function in vitro." Circ Shock 19:1-12, 1986

Rosenberg JC, Lillehei RC, Longerbeam J, Zimmerman B. "Studies on hemorrhagic and endotoxin shock in relation to vasomotor changes and endogenous circulating epinephrine, norepinephrine and serotonin." Ann Surg 154:611-627, 1961

Ross EM. "Pharmacodynamics: mechanisms of drug action and relationship between drug and concentration effect." in Goodman and Gillman's the Pharmacologic Basis of Therapeutics, $9^{\text {th }}$ ed. Hardman JG, Limbard LE, Molinoff PB, Ruddon R, Gilman AG eds. New York: McGraw Hill, 1996

Ross J, Linhart JW, Braunwald E. "Effects of changing heart rate in man by electrical stimulation of the right atrium - studies at rest, during exercise and with isoproterenol." Circulation 32:549-558, 1965

Rubanyi G, Kovach AG. "Effect of pentobarbital anesthesia on contractile performance and oxygen consumption of perfused rat heart." Circ Shock 7:121-127, 1980

Ruskoaho H, Tholken H, Lang RE. "Increase in atrial pressure releases atrial natriuretic peptide from isolated perfused rat hearts.” Pflugers Arch 407:170-174, 1986.

Sage MD, West EJ, Gavin JB. "Cardiac performance of isolated beating heart obtained from rats anesthetized by three different agents." Lab Anim Sci 35:153-155, 1985

Schaefer ME, Allert JA, Adams HR, Laughlin MH. "Adrenergic responsiveness and intrinsic sinoatrial automaticity of exercise-trained rats." Med Sci Sports Exerc 24:887-894, 1992 
Schlant R and Sonnenblick EH. "Normal physiology of the cardiovascular system." In The Heart, Arteries and Veins $8^{\text {th }}$ edition. Eds Schlant RC and Alexander RW. pp 113154,1994

Schmidlin OS, Bharati S, Lev M and Schwartz JB. "Effects of physiological aging on cardiac electrophysiology in perfused Fischer 344 rat hearts." Am J Physiol 262:H97-H105, 1992

Schmidt U, Hajjar RJ, Gwatheny JK. "The force-interval relationship in human myocardium.” J Card Fail 1:311-321, 1995

Schornack PA, Song SK, Hotchkiss R and Ackerman JH. "Inhibition of ion transport in septic rat heart: ${ }^{133} \mathrm{CS}+$ as an NMR active K+ analog." Am J Physiol 272:C1635C1641, 1997

Seed WA. "Interval-force effects in intact hearts." In The Interval Force Relationship of the Heart: Bowditch Revisited ed Noble MIM and Seed WA. 317-354 Cambridge, UK: University Press, 1992

Seeman B. The River of Life: The Story of Man's Blood From Magic to Science. New York: WW Norton and Company, Inc., 1961

Segal J, Schwalb H, Shmorak V, Uretzky G. "Effect of anesthesia on cardiac function and response in the perfused rat heart." J Mol Cell Cardiol 22:1317-1324, 1990

Shapiro R-, Breslow MJ, Miller C, Traystman RJ. "Sepsis induced coronary vasodilitation." Circ Shock 16:79-85, 1985

Sharma AC, Bosmann HB, Motew SJ, et al. "Steroid hormone alterations following induction of chronic intraperitoneal sepsis in male rats." Shock 6:150-154, 1996

Shepherd RE, McDonough KH, Burns AH. "Mechanism of cardiac dysfunction in hearts from endotoxin-treated rats." Circ Shock 19:371-384, 1986

Shepherd RE, Lang $\mathrm{CH}$, McDonough KH. "Myocardial adrenergic responsiveness after lethal and nonlethal doses of endotoxin." Am J Physiol 252:H410-H416, 1987

Shoemaker WC, Appel PL, Kram HB, Bishop MH, Abraham E. "Sequence of physiologic patterns in surgical septic shock." Crit Care Med 21:1876-1889, 1993

Siegel JH, Greenspan M, Del Guercia LRM. "Abnormal vascular tone, defective oxygen transport, and myocardial failure in human septic shock." Ann Surg 165:504-517, 1967

Silverman HJ, Lee NH, el-Fakahany EE. "Effects of endotoxin shock on lymphocytic betaadrenergic receptors." Circ Shock 32:293-306, 1990 
Smith LW, Winberry SL, Barker LA and McDonough KH. "Cardiac function and chronotropic sensitivity to $\beta$-adrenergic stimulation in sepsis." Am J Phys 251:H405H412, 1986

Smith LW and McDonough KH "Inotropic sensitivity to $\beta$-adrenergic stimulation in early sepsis." Am J Physiol 255:H699-H703, 1988

Soato GG and Krieger EM. "Heart rate after acute hypertension in the rat." Am J Physiol 227:1389-1393, 1974

Solis RT and Downing SE. "Effects of E. coli endotoxemia on ventricular performance." Am J Physiol 211:307-313, 1966

Spink WW, Reddin J, Zak SJ, et. al. "Correlation of plasma catecholamine levels with hemodynamic changes in endotoxin shock.” J Clin Invest 45:78-85, 1966

Spitzer JA. "Animal models of endotoxemia and sepsis." in Pathophysiology of shock, sepsis and organ failure Schlag G, Redl H eds. 1108-1118 New York: SpringerVerlag, 1993

Stein PK, Bosner M, Kleiger RE. "Heart rate variability: a measure of cardiac autonomic tone.” Am Heart J 127:1376-1381, 1993

Sutton JR, Cole A, Gunning J, Hickie JB and Seldon WA. "Control of heart rate in healthy young men." Lancet 1:1398-1400, 1967

Sugi K, Newald J, Traber LD, et. al. "Cardiac dysfunction after acute endotoxin administration $n$ conscious sheep." Am J Physiol 260:H1474-H1481, 1991

Tang C and Liu MS. "Initial externalization followed by internalization of beta-adrenergic receptors in rat heart during sepsis." Am J Physiol 270:R254-R263, 1996

Tang C, Hsu HK, Chen XY and Liu MS. "Externalization and internalization of $(\mathrm{Na}++\mathrm{K}$ + )-ATPase in rat heart during different phases of sepsis." Circ Shock 41:19-25, 1993

Tasken K, Skalhegg BS, Tasken KA, et. al. "Structure, function, and regulation of human cAMP-dependent protein kinases." Adv Second Messenger Phosphoprotein Res 31:191-204, 1997.

Thiemermann C. "Nitric oxide and septic shock." Gen Pharmacol 28:159-166, 1997

Toth I and Heard SO. "Nitric oxide does not mediate lipopolysaccharide induced myocardial depression in guinea pigs." Crit Care Med 25:684-688, 1997 
Tucker DC and Johnson AK. "Development of autonomic control of heart rate in genetically hypertensive and normotensive rats." Am J Physiol 246:R570-R577, 1984

Tukey JW. "Comparing individual means in the analysis of variance." Biometrics 5:99-114, 1949

Ulevitch RJ and Tobias PJ. "Receptor dependent mechanisms of cell stimulation by bacterial endotoxin.” Annu Rev Immunol 13:437-457, 1995

Van Oosterhout AJ, Van Heuven-Nolsen D, De Boer SF, Thijssen JH, Nijkamp FP. "Endotoxin-induced reduction of beta-adrenergic binding sites on splenic lymphocytes in vivo and in vitro: its modulation by anterior hypothalamic lesions." Life Sci 44:57-65, 1989

Vatner SF and Braunwald E. "Cardiac frequency: control and adjustments to alteration." Prog Cardiovasc Dis 14:431-445, 1972

Vatner SF, Monroe RG, McRitchie RJ. "Effects of anesthesia, tachycardia and autonomic blockade on the Anrep effect in intact dogs.” Am J Physiol 266:1450-1456, 1974

Waisbren BA. "Bacteremia due to gram-negative bacilli other than salmonella. Arch Intern Med 88:467-472, 1951

Walsh RR. "Heart rate and its neural regulation with rising body temperature in anesthetized rats." Am J Physiol 217:1139-1142, 1969

Wang SY, VanderMeer TJ, Fink MP, Selke FW. "Uncoupling of coronary microvascular ß2-adrenoceptors by Escherichia coli endotoxemia." Surgery 116:307-312, 1994

Webster C. "William Harvey's conception of the heart as a pump." Bull Hist Med 39:508517,1965

Weil MH and Nishijima H. "Cardiac output in bacterial shock." Am J Med 64:920-922, 1978

Weiss HR, Sadoff JD, Scholz PM, Klabunde RE. "Nitric oxide reduces myocardial contractility in isoproterenol stimulated rat hearts by a mechanism independent of cyclic GMP or cAMP.” Pharmacology 55:202-210, 1997

Werdan K, Muller U, Reithmann C. "Mechanisms in acute septic cardiomyopathy: evidence from isolated myocytes." Basic Res Cardiol 86:411-421, 1991.

Westerhoff $\mathrm{N}$ and Elzinga G. "Why smaller animals have higher heart rates." In Interactive Phenomena in the Cardiac System eds Sideman S and Beyar R. 319-323 New York: Plenum Press, 1993 
Weyrich AS, Ma XL, Buerke M, et. al. "Physiological concentrations of nitric oxide do not elicit an acute negative inotropic effect in unstimulated cardiac muscle." Circ Res 75:692-700, 1994

Whitmarsh AJ and Davis RJ. "Transcription factor AP-1 regulation by mitogen activated protein kinase signal transduction pathways.” J Mol Med 74:589-607, 1996

Wilcken DEL, Shorey DCD, MacDonald GJ. "Reserpine: effects on structure of heart muscle." Science 157:1332-1334, 1967

Woodworth RS. "Maximal contraction, 'staircase' contraction, refractory period, and compensatory pause of the heart." Am J Physiol 8: 213-249, 1902

Wu LL and Liu MS. "Impaired calcium uptake by cardiac sarcoplasmic reticulum and its underlying mechanism in endotoxin shock." Mol Cell Biochem 108:9-17, 1991

Wu SN, Lue SI, Yang SL, Hsu HK, Liu MS. "Electrophysiologic properties of isolated adult cardiomyocytes from septic rats." Circ Shock 41:239-247, 1993

Yasuda S and Lew YY. "Lipopolysaccharide depresses cardiac contractility and betaadrenergic contractile response by decreasing myofilament response to $\mathrm{Ca} 2+$ in cardiac myocytes." Circ Res 81:1011-1120, 1997

Yatani A, Okabe K, Codina J, Birnbaumer L, Brown AM. "Heart rate regulation by G proteins acting on the cardiac pacemaker channel." Science 249:1163-1166, 1990

Yu P, Boughner DR, Sibbald WJ, et. al. "Myocardial collagen changes and edema in rats with hyperdynamic sepsis." Crit Care Med 25:657-662, 1997

Zetterstrom BEM, Palmerio C, Fine J. "Protection of functional and vascular integrity of the spleen in traumatic shock by denervation.” Proc Soc Exp Biol 117:373-376, 1964

Zhou ZZ, Wurster RD, Qi M, Jones SB. "Sympathoadrenal activation in sinoaortic denervated rats following endotoxin.” Am J Physiol 260:R739-R746, 1991

Zhu Y, Yang HT, Endoh M. "Negative chronotropic and inotropic effects of endothelin isopeptides in mammalian cardiac muscle.” Am J Physiol 273:H119-127, 1997 
Peter D. Wearden,

\section{Peter D. Wearden, MD, PhD}

\section{$\underline{\text { PERSONAL }}$}

Address: $\quad$ PO Box 9238

Department of Surgery

Robert C. Byrd Health Sciences Center

West Virginia University

Morgantown, WV 26506-9238

Telephone: Work (304) 293 - 3311 Home (304) 599 - 6739

email: $\quad$ pwearden@wvu.edu

Date of Birth: July 18, 1966 Monongalia County, WV

Spouse: $\quad$ Andrea

Children: $\quad$ Andrew Peter (07/24/93); Mara Louise (10/14/95); Luke John Paul

(04/08/98)

\section{$\underline{\text { EDUCATION }}$}

1999 PhD., Pharmacology and Toxicology, School of Medicine, West Virginia University, Morgantown, WV. Dissertation Title - "Alterations in Intrinsic Heart Rate in Endotoxemia." Major Professors - WW Fleming, Ph.D., Professor and Chairman; DA Taylor, Ph.D, Professor

1993 M.D., West Virginia University, Morgantown, WV

1988 A.B., Government with honors, Georgetown University, Washington, D.C. Honors Thesis - "The Role of Leadership in Transitions to Democracy."

\section{CERTIFICATION}

1999 ATLS

1994 National Board of Medical Examiners

\section{$\underline{\text { LICENSURE }}$}

1998 West Virginia State Board of Medicine

\section{EXPERIENCE}

1998- DEPARTMENT OF SURGERY, West Virginia University School of Medicine Junior Surgical Resident

Responsible for the care of surgical patients at a PGY 3 level at the Ruby Memorial (University) Hospital

1995- DEPARTMENT OF SURGERY, West Virginia University School of Medicine 1998 Research Associate in Surgery

Research projects include an examination of myocardial function in sepsis utilizing in vivo, in vitro and isolated myocyte techniques. 
Peter D. Wearden,

1993- DEPARTMENT OF SURGERY, West Virginia University School of Medicine

1995 Junior Surgical Resident

Responsible for the care of surgical patients at a junior resident level at the Ruby Memorial (University) Hospital and L.A. Johnson V.A. Medical Center.

1988- DEPARTMENT OF ANATOMY, West Virginia University School of Medicine

1990 Research Assistant $\quad$ Laboratory of R.D. Dey

Assisted in all aspects of research projects including development of grant proposals and protocol, experimental procedures and statistical analysis. Skills included light and electron microscopy, cell culture, immunocytochemistry, microdissection, microinjection, stereometry and computer programming.

\section{$\underline{\text { HONORS }}$}

1998 Region III first place (basic science), American College of Surgeons Residents' Trauma Paper Competition.

1998 State (WV) first place, American College of Surgeons Residents' Trauma Paper Competition.

1998 Second Place, 10th Annual Bernard Zimmerman Resident Research Competition, Department of Surgery, West Virginia University

1997 First Place, 9th Annual Bernard Zimmerman Resident Research Competition, Department of Surgery, West Virginia University

1996 Alpha Omega Alpha, Honor Medical Society

1996 First place - national, clinical paper, American College of Surgeons, Committee on Trauma, Residents' Trauma Paper Competition, 74th annual meeting, Minneapolis, MN.

1996 Second Place, 8th Annual Bernard Zimmerman Resident Research Competition, Department of Surgery, West Virginia University.

1995 Region III first place, American College of Surgeons Residents' Trauma Paper Competition.

1995 State (WV) first place, American College of Surgeons Residents' Trauma Paper Competition.

1994 Third place, West Virginia University Department of Surgery Junior Resident Paper Competition.

1994 State (WV) second place, American College of Surgeons Residents' Trauma Paper Competition.

1993 Outstanding Graduate, West Virginia University School of Medicine.

1993 E. J. Van Liere Research Award, West Virginia University School of Medicine.

1993 Elected to Mountain West Virginia University ranking honorary.

\section{$\underline{\text { SERVICE }}$}

1998

1997

1996 -

$1996-$

$1994-1996$
Ruby Memorial Hospital House Staff Coordinator Search Committee Patient Centered Committee, West Virginia University School of Medicine West Virginia University School of Medicine Coordinating Committee for Graduate Medical Education, Resident Representative

Department of Surgery Research Committee

Department of Surgery Curriculum Committee 
Peter D. Wearden,

1992 - 1993 Chair, Committee for Student Report to LCME Accreditation Team

1992 - 1993 School of Medicine Admissions Committee

1991 - 1992 Executive Council Member, Medical Student Section, WV State Medical Association

$1991 \quad$ Rural Health Care Conference Planning Committee

1990 - 1992 President, WVU Medical Student Society

1989 - 1993 School of Medicine Curriculum Review Committee, Medical Student

Representative

\section{$\underline{\text { PRESENTATIONS }}$}

1999 "Alterations in Intrinsic Heart Rate in Endotoxemia” Ph.D. Dissertation Seminar, School of Medicine, West Virginia University

1999 "Clinical Correlation - Anatomy of the Abdomen" Department of Anatomy, First Year Medical Class, School of Medicine, West Virginia University.

1998 "Alterations in Heart Rate Exhibit a Different Time Course Than Other Hemodynamic Changes in Rat Endotoxemia." American College of Surgeons Committee on Trauma Resident Paper Competition.

1998 "The Regulation of Heart Rate in Endotoxemia.” Department of Surgery, Grand Rounds, West Virginia University.

1998 "Tachycardia in Rat Endotoxemia Exhibits a Different Time Course than Alterations in Contractility." 10th Annual Bernard Zimmerman Resident Research Competition.

1997 "Endotoxin and Cardiac Function: Tachycardia at the Heart (of the Matter)" Department of Pharmacology and Toxicology, West Virginia University.

1997 “Tachycardia in Endotoxemia is Abolished By Reserpine Pretreatment but not Propranolol Treatment." 9th Annual Bernard Zimmerman Resident Research Competition.

1996 “The Regulation of Heart Rate.” Department of Surgery, Grand Rounds, West Virginia University.

1996 "Evidence for the Nonautonomic Regulation of Heart Rate in Sepsis." 8th Annual Bernard Zimmerman Resident Research Competition.

1995 "The Role of Bronchoscopic and Nonbronchoscopic Techniques in the Diagnosis of Ventilator Associated Pneumonia." American College of Surgeons Committee on Trauma Resident Paper Competition.

1994 "Hypothermia and Hyperamylasemia in the Multiply Injured Trauma Patient." American College of Surgeons Committee on Trauma Resident Paper Competition. 
1994 Jones DR, Wearden PD, Kalantarian B, et al. "Diagnosis and Management of Boerhaave's Syndrome." American College of Chest Physicians Poster Presentation.

1993 Wearden PD, Jones DR, Vasilakis A. "Diverticulitis in Patients Less Than Age Forty: Analysis of 411 Patients." American College of Surgeons Clinical Congress Scientific Exhibit.

1993 Price RA, Cmolik B, Wearden PD, Hrabovsky EE. "Correction of Chest Wall Defect in a Neonate with Poland's Syndrome." American College of Surgeons Clinical Congress Scientific Exhibit.

1993 Nelson MG, Jones DR, Wearden PD, Graeber GM. “Clinical Manifestations of Marphan Syndrome." American College of Surgeons Clinical Congress Scientific Exhibit.

1993 "Morphologic Changes in the Alveolar Walls of Rats Exposed to Fresh and Stale Coal Dust.” E.J. Van Liere Research Convocation.

\section{$\underline{\text { PUBLICATIONS }}$}

1996 Wearden PD, Chendrasekhar A, Timberlake GA. "The role of nonbronchoscopic techniques in the diagnosis of ventilator associated pneumonia." J Trauma 41:703-707

1995 Wearden PD, Hill RC, Hollingsed M, et al. "Successful case of extracorporeal life support in a patient with postcardiotomy cardiogenic shock." W Va Med Jour 91:57-58

1994 Jones DR, Wearden PD, Kalantarian B, et al. "Diagnosis and management of Boerhaave's Syndrome."

1994 Jones DR, Vasilakis A, Wearden PD, et al. "Technique for management of an axilloaxillary by-pass graft complicating median sternotomy." J Cardiovasc Surg 35:235237

1991 Reist R, Bryner K, Wearden PD, et al. "Development of a bioassay for pulmonary cell production of fibrogenic factors." Toxicology Methods 1:53-65 